\title{
Motion planning and feedback control techniques with applications to long tractor-trailer vehicles
}

\section{Oskar Ljungqvist}

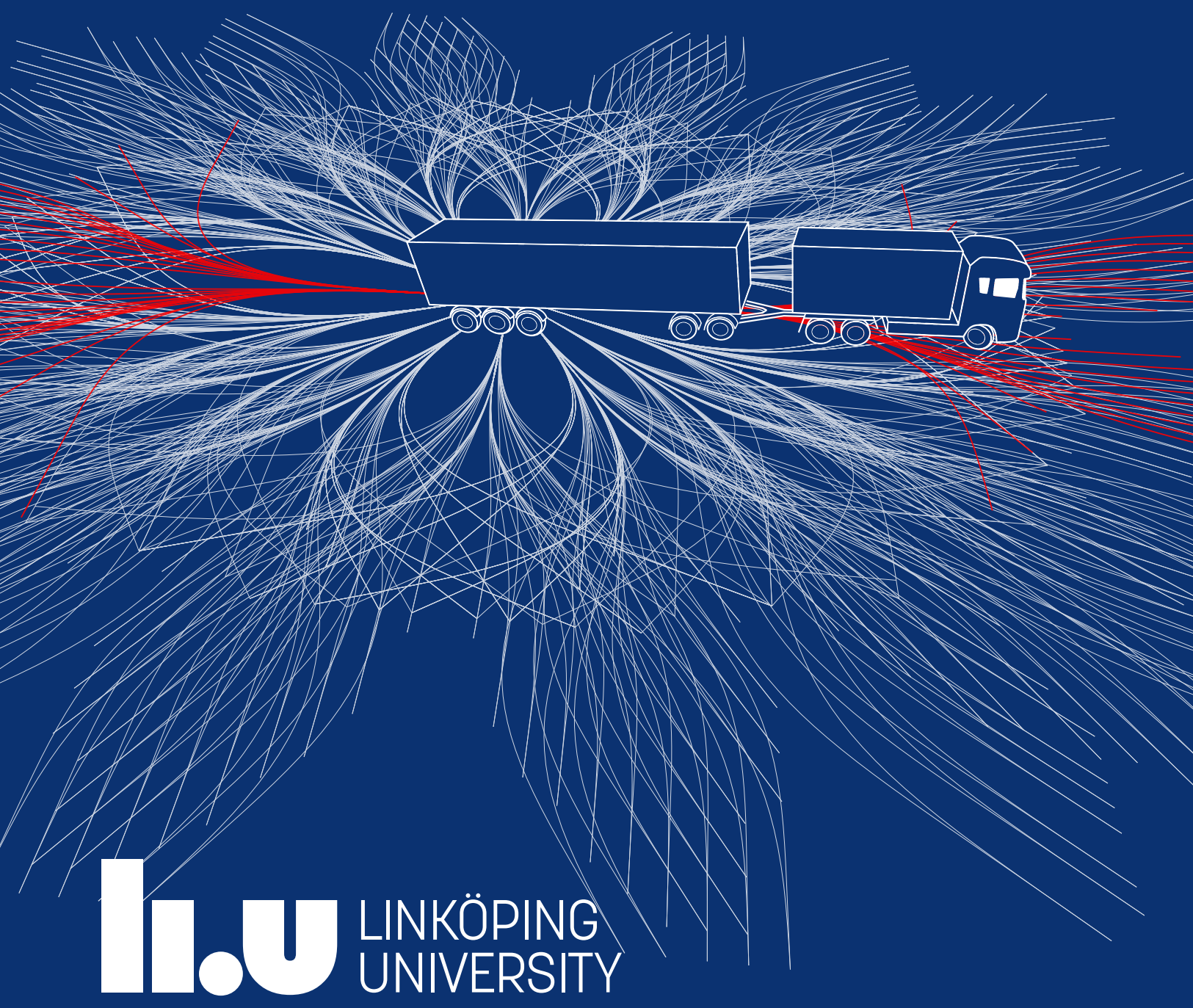


Linköping studies in science and technology. Dissertations.

No. 2070

Motion planning and

feedback control techniques

with applications to long

tractor-trailer vehicles

Oskar Ljungqvist 
Cover illustration: A library of optimized manuevers for a tractor-trailer vehicle.

Linköping studies in science and technology. Dissertations.

No. 2070

Motion planning and feedback control techniques with applications to long tractor-trailer vehicles

Oskar Ljungqvist

oskar. ljungqvist@liu.se

www. control.isy.liu.se

Division of Automatic Control

Department of Electrical Engineering

Linköping University

SE-581 83 Linköping

Sweden

ISBN 978-91-7929-858-6

ISSN 0345-7524

Copyright (C) 2020 Oskar Ljungqvist

Printed by LiU-Tryck, Linköping, Sweden 2020 
To my family! 



\section{Abstract}

During the last decades, improved sensor and hardware technologies as well as new methods and algorithms have made self-driving vehicles a realistic possibility in the near future. At the same time, there has been a growing demand within the transportation sector to increase efficiency and to reduce the environmental impact related to transportation of people and goods. Therefore, many leading automotive and technology companies have turned their attention towards developing advanced driver assistance systems and self-driving vehicles.

Autonomous vehicles are expected to have their first big impact in closed environments, such as mines, harbors, loading and offloading sites. In such areas, the legal requirements are less restrictive and the surrounding environment is more controlled and predictable compared to urban areas. Expected positive outcomes include increased productivity and safety, reduced emissions and the possibility to relieve the human from performing complex or dangerous tasks. Within these sites, tractor-trailer vehicles are frequently used for transportation. These vehicles are composed of several interconnected vehicle segments, and are therefore large, complex and unstable while reversing. This thesis addresses the problem of designing efficient motion planning and feedback control techniques for such systems.

The contributions of this thesis are within the area of motion planning and feedback control for long tractor-trailer combinations operating at low-speeds in closed and unstructured environments. It includes development of motion planning and feedback control frameworks, structured design tools for guaranteeing closed-loop stability and experimental validation of the proposed solutions through simulations, lab and field experiments. Even though the primary application in this work is tractor-trailer vehicles, many of the proposed approaches can with some adjustments also be used for other systems, such as drones and ships.

The developed sampling-based motion planning algorithms are based upon the probabilistic closed-loop rapidly exploring random tree (CL-RRT) algorithm and the deterministic lattice-based motion planning algorithm. It is also proposed to use numerical optimal control offline for precomputing libraries of optimized maneuvers as well as during online planning in the form of a warm-started optimization step.

To follow the motion plan, several predictive path-following control approaches are proposed with different computational complexity and performance. Common for these approaches are that they use a path-following error model of the vehicle for future predictions and are tailored to operate in series with a motion planner that computes feasible paths. The design strategies for the path-following approaches include linear quadratic (LQ) control and several advanced model predictive control (MPC) techniques to account for physical and sensing limitations. To strengthen the practical value of the developed techniques, several of the proposed approaches have been implemented and successfully demonstrated in field experiments on a full-scale test platform. To estimate the vehicle states needed for control, a novel nonlinear observer is evaluated on the full-scale test vehicle. It is designed to only utilize information from sensors that are mounted on the tractor, making the system independent of any sensor mounted on the trailer. 



\section{Populärvetenskaplig sammanfattning}

Under de senaste årtiondena har utvecklingen av sensor- och hårdvaruteknik gått i en snabb takt, samtidigt som nya metoder och algoritmer har introducerats. Samtidigt ställs det stora krav på transportsektorn att öka effektiviteten och minska miljöpåverkan vid transporter av både människor och varor. Som en följd av detta har många ledande fordonstillverkare och teknikföretag börjat satsat på att utveckla avancerade förarstödsystem och självkörande fordon. Även forskningen inom autonoma fordon har under de senaste årtiondena kraftig ökat då en rad tekniska problem återstår att lösas.

Förarlösa fordon förväntas få sitt första stora genombrott i slutna miljöer, såsom gruvor, hamnar, lastnings- och lossningsplatser. I sådana områden är lagstiftningen mindre hård jämfört med stadsområden och omgivningen är mer kontrollerad och förutsägbar. Några av de förväntade positiva effekterna är ökad produktivitet och säkerhet, minskade utsläpp och möjligheten att avlasta människor från att utföra svåra eller farliga uppgifter. Inom dessa platser används ofta lastbilar med olika släpvagnskombinationer för att transportera material. En sådan fordonskombination är uppbyggd av flera ihopkopplade moduler och är således utmanande att backa då systemet är instabilt. Detta gör det svårt att utforma ramverk för att styra sådana system vid exempelvis autonom backning.

Självkörande fordon är mycket komplexa system som består av en rad olika komponenter vilka är designade för att lösa separata delproblem. Två viktiga komponenter i ett självkörande fordon är dels rörelseplaneraren som har i uppgift att planera hur fordonet ska röra sig för att på ett säkert sätt nå ett överordnat mål, och dels den banföljande regulatorn vars uppgift är att se till att den planerade manövern faktiskt utförs i praktiken trots störningar och modellfel. I denna avhandling presenteras flera olika algoritmer för att planera och utföra komplexa manövrar för lastbilar med olika typer av släpvagnskombinationer. De presenterade algoritmerna är avsedda att användas som avancerade förarstödsystem eller som komponenter i ett helt autonomt system. Även om den primära applikationen i denna avhandling är lastbilar med släp, kan många av de förslagna algoritmerna även användas för en rad andra system, så som drönare och båtar.

Experimentell validering är viktigt för att motivera att en föreslagen algoritm är användbar i praktiken. I denna avhandling har flera av de föreslagna planeringsoch reglerstrategierna implementerats på en småskalig testplattform och utvärderats i en kontrollerad labbmiljö. Utöver detta har även flera av de föreslagna ramverken implementerats och utvärderats i fältexperiment på en fullskalig testplattform som har utvecklats i samarbete med Scania CV. Här utvärderas även en ny metod för att skatta släpvagnens beteende genom att endast utnyttja information från sensorer monterade på lastbilen, vilket gör det föreslagna ramverket oberoende av sensorer monterade på släpvagnen. 



\section{Acknowledgments}

I would like to start express my sincere gratitude to my supervisor Daniel Axehill for his excellent guidance and encouragement throughout my $\mathrm{PhD}$ journey. Thank you for our many discussions and your never-ending enthusiasm and support. I would also like to direct a special thanks to Niclas Evestedt and Kristoffer Bergman for fantastic collaborations in several projects during the past years.

I am very grateful for valuable collaboration and support from Anders Helmersson and my co-supervisor Johan Löfberg. Financial support from the Strategic vehicle research and innovation progamme (FFI) (Contract number: 2017-01957) and Scania CV are hereby gratefully acknowledged. Also, thank you Svante Gunnarsson for inviting me to be part of the Automatic control group and for your excellent work as the former Head of the Division of Automatic Control. Thank you Martin Enqvist for keeping up a nice and friendly working climate as the new Head of the Division. Thank you Ninna Stensgård for helping me with administrative things and for making sure everything runs smoothly in the group.

I gratefully acknowledge the help from Gustaf Hendeby regarding AATEX is- $_{\mathrm{E}}$ sues and for providing the $\mathrm{AAT}_{\mathrm{E} X} \mathrm{X}$-class that has been used to write this thesis. I also appreciate the help from Kristoffer Bergman, Per Boström-Rost, Daniel Arnström, Martin Lindfors and Anders Helmersson for proof reading the thesis and contributed with suggestions to improve it. All remaining errors are my own.

During my time as a PhD student at the Division of Automatic Control I have gained a lot of new and wonderful friends. A special thanks to Johan Dahlin and Andreas Bergström for all our refreshing lunch runs. Johan, thank you for your guidance during the beginning of my $\mathrm{PhD}$ studies, for being a good friend, and for encouraging me to prioritize lunch runs, even during periods with high workload. Thank you Kristoffer Bergman and Per Boström-Rost for all our productive discussions and collaboration over the past years, for bearing with my humble side, and for making the best out of the international WASP trips. I really hope that I will have the opportunity to continue collaborating with you guys in the future. I would also like to express my gratitude to Mattias Tiger and Olov Andersson at the Department of Computer and Information Science for fruitful collaboration that was initiated during the WASP project course. Thank you Daniel Simon and Isak Nielsen for an unforgettable road trip in the USA, where earning (losing) money at casinos, visiting Grand Canyon and saving the life of an injured stranger from a sandstorm in Death Valley are some of the highlights from the trip. Thank you everyone else from the Automatic Control group for making this a stimulating environment!

I am also grateful to Scania CV and the Autonomous Transport Solutions for long and fruitful collaboration. A special thanks to past and present members of the Autonomous Motion team including Henrik Pettersson, Lars Hjort, Marcello Cirillo, Assad Alam, Michael Åström, Christoffer Norén, Pedro Lima, Rui Oliveira, Goncalo Collares Pereira, Christian Larsson, Laura Dal Col and many more. Thank you Marcello and Rui for excellent research collaborations over the past years, which have resulted in many interesting new insights. A special thanks is directed to Henrik for learning me about classic sports cars and for widening 
my music taste during long days at Scania's test track.

Many thanks to my family for their encouragement, enthusiasm and support. Last but not the least, to Marie and our dogs, thank you for bearing with me during my $\mathrm{PhD}$ journey, for all your love, for all wonderful adventures we have done together and for those to come.

Linköping, April 2020

Oskar Ljungqvist 


\section{Contents}

\section{Background}

1 Introduction $\mathbf{3}$

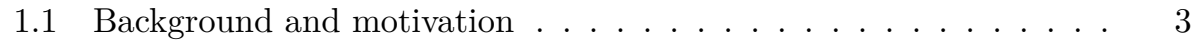

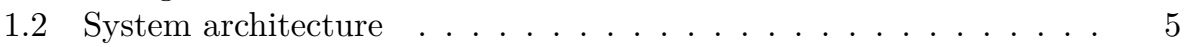

1.3 Main contributions . . . . . . . . . . . . . . . . . . . 7

1.4 Thesis outline and contributions . . . . . . . . . . . . 8

1.5 Other publications .................... 12

2 Modeling of wheeled vehicles 15

2.1 Introduction . . . . . . . . . . . . . . . . . . . . 15

2.2 Nonholonomic systems . . . . . . . . . . . . . . . . . . . 16

2.3 The kinematic bicycle model . . . . . . . . . . . . . . . 17

2.4 The general N-trailer with a car-like tractor . . . . . . . . . . . 19

3 Motion planning for self-driving vehicles 25

3.1 Introduction . . . . . . . . . . . . . . . . . 25

3.2 Problem formulation . . . . . . . . . . . . . . . . . . 26

3.3 A common solution concept ................. . . 28

3.4 Nonholonomic motion planning . . . . . . . . . . . . . . . . . 29

3.4.1 Steering functions . . . . . . . . . . . . . . . 29

3.4 .2 Heuristics . . . . . . . . . . . . . . . . . . . . . . . . 32

3.4.3 Collision detection ................... 34

3.5 Sampling-based motion planning . . . . . . . . . . . . . . . . . 35

3.5.1 Motion planning in state lattices . . . . . . . . . . . . . 37

3.5.2 Motion planning using RRT . . . . . . . . . . . . 43

4 Feedback control for self-driving vehicles $\quad 45$

4.1 Problem formulations . . . . . . . . . . . . . . . . 45

4.2 Trajectory-tracking control ................ 47 
4.2.1 Linear trajectory-tracking techniques . . . . . . . . . . 48

4.2.2 Robust control design using linear matrix inequalities . . . 50

4.2.3 Linear quadratic control . . . . . . . . . . . . . . 51

4.2.4 Model predictive control . . . . . . . . . . . . . . 53

4.2.5 Trajectory-tracking control using LQ control: An example . 54

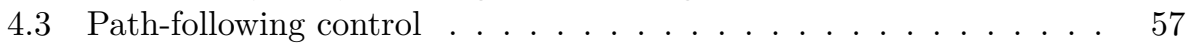

4.3.1 Pure-pursuit controller . . . . . . . . . . . . . 59

4.3.2 Nonlinear path-following techniques . . . . . . . . . . . 61

4.3.3 Path-following control using LQ control: An example . . . 62

5 Concluding remarks $\quad 67$

5.1 Summary of contributions . . . . . . . . . . . . . . . . 67

5.2 Future work . . . . . . . . . . . . . . . . . . . 69

$\begin{array}{ll}\text { A Derivation of the tracking-error system } & \mathbf{7 5}\end{array}$

$\begin{array}{ll}\text { Bibliography } & 77\end{array}$

\section{Publications}

A Motion planning for a reversing general 2-trailer configuration using Closed-Loop RRT $\quad 93$

1 Introduction . . . . . . . . . . . . . . . . . . . 95

1.1 Related work . . . . . . . . . . . . . 96

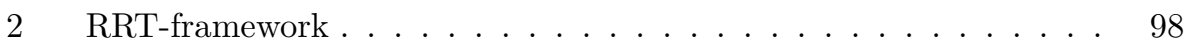

2.1 Tree expansion . . . . . . . . . . . . . . . . . . . . . 99

3 System dynamics . . . . . . . . . . . . . . . . . . . . . . . 100

4 Stabilization and path tracking . . . . . . . . . . . . . . 101

$4.1 \quad$ LQ controller . . . . . . . . . . . . . . . . . . 101

4.2 Path tracking . . . . . . . . . . . . . . . 103

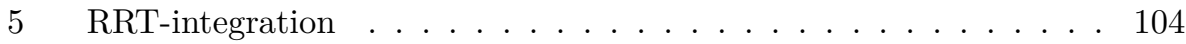

5.1 Node connection heuristic . . . . . . . . . . . . 104

5.2 Goal evaluation . . . . . . . . . . . . . . . . 104

5.3 Cost function . . . . . . . . . . . . . . . . . . . 104

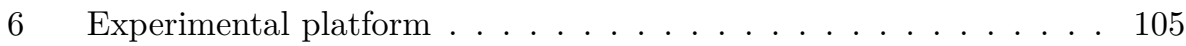

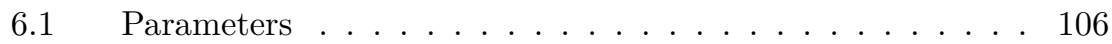

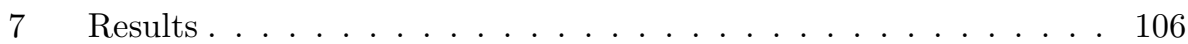

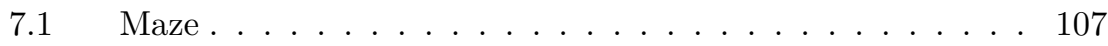

7.2 Two-point turn . . . . . . . . . . . . . . 107

$7.3 \quad$ Driver test . . . . . . . . . . . . . . . 109

7.4 Real-world experiments ............... . . 111

8 Conclusions and future work . . . . . . . . . . . . . 111

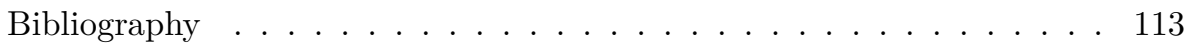

B Path following control for a reversing general 2-trailer system $\mathbf{1 1 5}$

1 Introduction . . . . . . . . . . . . . . . . 117 
1.1 Related work . . . . . . . . . . . . . . 118

2 Modeling ........................ 119

2.1 Frenet frame . . . . . . . . . . . . . . . 120

$2.2 \quad$ Linearization around paths . . . . . . . . . . . . 122

3 Stabilization . . . . . . . . . . . . . . . . 123

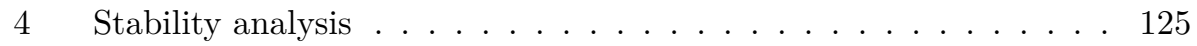

5 Results . . . . . . . . . . . . . . . . 127

5.1 Stability around a set of paths . . . . . . . . . . . 127

5.2 Simulation results . . . . . . . . . . . . . . . . 128

6 Conclusions and future work . . . . . . . . . . . . . . . 129

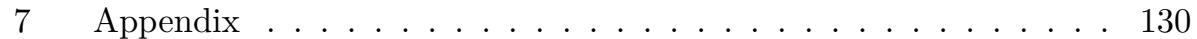

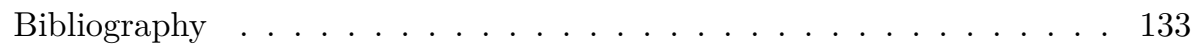

C On stability for state-lattice trajectory tracking control $\quad \mathbf{1 3 5}$

1 Introduction . . . . . . . . . . . . . . . . . 137

1.1 Related work . . . . . . . . . . . . . 139

2 The lattice planner framework . . . . . . . . . . . . . . . . . 140

3 Connection to hybrid systems . . . . . . . . . . . . . . . . . 141

4 Low-level controller synthesis . . . . . . . . . . . . . . . . . . . . . 141

5 Convergence along a combination of motion primitives . . . . . . . 144

6 Application results . . . . . . . . . . . . . . . . . 147

6.1 The lattice planner . . . . . . . . . . . . . . . . . 147

6.2 Low-level controller synthesis . . . . . . . . . . . . . . . . . 149

6.3 Analyzing the closed-loop hybrid system . . . . . . . . . . 152

6.4 Simulation results ................ . . 153

7 Conclusions and future work . . . . . . . . . . . . . . . . 154

Bibliography ...................... 156

D A path planning and path-following control framework for a $\begin{array}{lr}\text { general 2-trailer with a car-like tractor } & 159\end{array}$

1 Introduction . . . . . . . . . . . . . . . . . . . 161

2 Background and related work . . . . . . . . . . . . . 163

2.1 Perception and localization . . . . . . . . . . . 163

2.2 State estimation . . . . . . . . . . . . . . . . 164

2.3 Path planning. . . . . . . . . . . . . . . 165

2.4 Path-following control . . . . . . . . . . . 167

3 Kinematic vehicle model and problem formulations . . . . . . . . . 169

3.1 Problem formulations . . . . . . . . . . . . . . 171

3.2 System properties . . . . . . . . . . . . . . . . . . 172

4 Lattice-based path planner . . . . . . . . . . . . . . . . . . . . . 174

4.1 State lattice construction . . . . . . . . . . . 176

4.2 Motion primitive generation . . . . . . . . . . . 178

4.3 Efficiency improvements and online path planning . . . . . 181

5 Path-following controller . . . . . . . . . . . . . . 181

5.1 Local behavior around a nominal path . . . . . . . . . . . 184

5.2 Connection to hybrid systems . . . . . . . . . . 185 
5.3 Design of the hybrid path-following controller . . . . . . 186

5.4 Convergence along a combination of motion primitives . . . 188

6 State observer . . . . . . . . . . . . . . . . . 190

$6.1 \quad$ Extended Kalman filter . . . . . . . . . . . . . . . . 191

7 Implementation details: Application to full-scale tractor-trailer system ........................... 194

$7.1 \quad$ Lattice planner . . . . . . . . . . . . . . . . . . . . 194

7.2 Path-following controller . . . . . . . . . . . . . 195

7.3 State observer . . . . . . . . . . . . . . 197

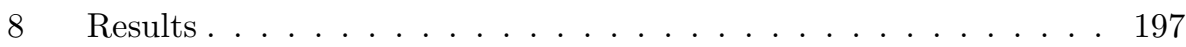

8.1 Analysis of the closed-loop hybrid system . . . . . . . . 197

8.2 Simulation results . . . . . . . . . . . . . 199

8.3 Results from real-world experiments . . . . . . . . . 205

8.4 Discussion of lessons learned . . . . . . . . . . . . 212

9 Conclusions and future work . . . . . . . . . . . . . . . 213

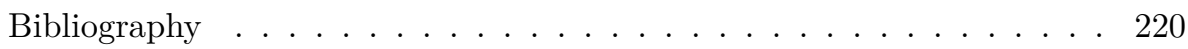

E Estimation-aware model predictive path-following control for a $\begin{array}{lr}\text { general 2-trailer with a car-like tractor } & 227\end{array}$

1 Introduction . . . . . . . . . . . . . . . . . 229

2 Vehicle model . . . . . . . . . . . . . . . . . . . . . 232

2.1 Constraints . . . . . . . . . . . . . . 233

2.2 Path-following error model . . . . . . . . . . . . 234

3 Model predictive path-following controller . . . . . . . . . . . 236

4 Estimation-aware controller design . . . . . . . . . . . . . 238

4.1 Design of cost function . . . . . . . . . . . . . . 239

4.2 Modeling of the constraint on the joint angles . . . . . . . . 240

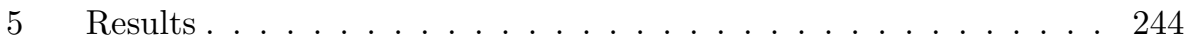

5.1 Simulation setup . . . . . . . . . . . . . . . 244

5.2 Simulation results . . . . . . . . . . . . 245

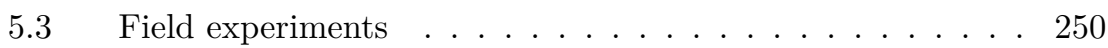

5.4 Results from field experiments . . . . . . . . . . . 250

6 Conclusions and future work . . . . . . . . . . . . 253

Bibliography .................... . . 256

F Optimization-based motion planning for multi-steered articulated vehicles

1 Introduction . . . . . . . . . . . . . . . . . . 263

2 Kinematic vehicle model and problem formulation . . . . . . . . 265

2.1 Problem formulation . . . . . . . . . . . . . 268

2.2 Trajectory planning framework . . . . . . . . . . . 268

3 Lattice-based trajectory planner . . . . . . . . . . . . . 270

3.1 State-space discretization . . . . . . . . . . . . . 271

3.2 Motion primitive generation . . . . . . . . . . . 271

4 Homotopy-based optimization step . . . . . . . . . . . . . . . . 273

5 Simulation results .................... 275 
6 Conclusions . . . . . . . . . . . . . . . . . 279

Bibliography ....................... 281

G A predictive path-following controller for multi-steered articulated vehicles 1 Introduction . . . . . . . . . . . . . . . . . . . . . 287

2 Kinematic vehicle model . . . . . . . . . . . . . . . . . . 288

3 Path-following error model . . . . . . . . . . . . . . . . . 291

4 Model predictive path-following controller . . . . . . . . . . . 293

$4.1 \quad$ Controller design . . . . . . . . . . . . . . . 295

5 Simulation results . . . . . . . . . . . . . . . . 296

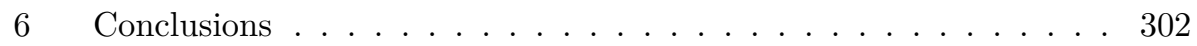

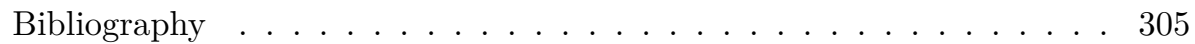





\section{Notation}

\section{Acronyms}

\begin{tabular}{cl}
\hline Acronyms & Meaning \\
\hline AABB & Axis-aligned bounding box \\
ADAS & Advanced driver assistance systems \\
ARA* & Anytime repairing A* \\
ARE & Algebraic Riccati equation \\
BVP & Boundary-value problem \\
CL-RRT & Closed-loop RRT \\
EKF & Extended Kalman filter \\
FFI & Fordonsstrategisk forskning och innovation \\
GNSS & Global navigation satellite system \\
GPS & Global positioning system \\
HLUT & Heuristic look-up table \\
IMU & Inertial measurement unit \\
LDI & Linear differential inclusion \\
LIDAR & Light detection and ranging \\
LMI & Linear matrix inequality \\
LPV & Linear parameter-varying \\
LQ & Linear quadratic \\
LTV & Linear time-varying \\
MIP & Mixed integer programming \\
MIQP & Mixed integer quadratic programming \\
MPC & Model predictive control \\
MSNT & Multi-steered N-trailer \\
NLP & Nonlinear programming \\
OBB & Oriented bounding box \\
OCP & Optimal control problem \\
PID & Proportional, integral, derivative \\
PMP & Pontryagin's minimum (maximum) principle \\
QP & Quadratic programming \\
RADAR & Radio detection and ranging \\
RRT & Rapidly-exploring random tree \\
SDP & Semidefinite programming \\
SQP & Sequential quadratic programming \\
SSNT & Single-steered N-trailer \\
\hline &
\end{tabular}




\section{Notation}

\begin{tabular}{cl}
\hline Notation & Meaning \\
\hline $\mathbb{R}$ & Set of real numbers \\
$\mathbb{R}_{+}$ & Set of positive real numbers \\
$\mathbb{Z}$ & Set of integers \\
$\mathbb{Z}_{+}$ & Set of positive integers \\
$\mathbb{Z}_{+}$ & Set of strictly positive integers \\
$\mathbb{R}^{n}$ & Set of real vectors with $n$ components \\
$\mathbb{R}^{n \times m}$ & Set of real matrices with $n$ rows and $m$ columns \\
$\mathbb{S}_{+}^{n}$ & Set of positive semidefinite matrices with $n$ columns \\
$\mathbb{S}_{++}^{n}$ & Set of positive definite matrices with $n$ columns \\
$A \succeq 0$ & Matrix $A$ is positive semidefinite \\
$A \succ 0$ & Matrix $A$ is positive definite \\
$\operatorname{det}(A)$ & Determinant of a matrix $A$ \\
$\operatorname{rank}(A)$ & Rank of a matrix $A$ \\
$\sigma_{\max }(\min )(A)$ & Maximum (minimum) singular value of matrix $A$ \\
$\operatorname{Cond}(A)$ & Condition number of matrix $A$ \\
$\operatorname{Co} P$ & Convex hull of a set of points $P$ \\
$|\mathcal{S}|$ & Cardinality of a set $\mathcal{S}$ \\
\hline
\end{tabular}



Part I

\section{Background}





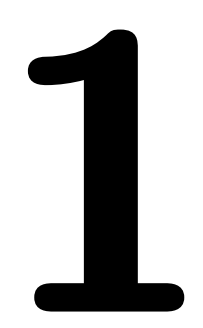

\section{Introduction}

This chapter introduces the research fields of self-driving vehicles and advanced driver assistance systems (ADAS). The chapter starts with a short background and motivation why research in this field is important. In Section 1.2, a simplified system architecture of a self-driving vehicle is presented and in Section 1.3, the main contributions of this thesis are summarized. Finally, Section 1.4 provides an overview of the contributions and an outline of the thesis.

\subsection{Background and motivation}

In the last decades, emerging sensor and hardware technologies have made the idea of developing self-driving vehicles a realistic possibility in the near future. Ever since the groundbreaking DARPA Grand Challenges (Buehler et al., 2007) and DARPA Urban Challenge (Buehler et al., 2009) were held, many leading automotive manufacturers and technology companies have turned their attention towards developing self-driving vehicles. Removing the human from the steering wheel and instead providing mobility as a service is predicted to have positive effects on road safety, reduced greenhouse gas emissions and enhanced utilization of the overall vehicle fleet (Burns, 2013). Car manufacturers see added value to their customers and the possibility to gain a competitive edge to their competitors by providing this technology.

At the same time, the transportation industry sees growing demands for delivering goods and transporting people. However, since the transportation industry is one of the largest emitter of greenhouse gases, the European Road Transport Research Advisory Council (2013) and the European Commission (2011) have set up strict goals for improvement of the European transportation system with an overall efficiency improvement by $50 \%$ in 2030 compared to 2010 while reducing emissions by $60 \%$. To fulfill these requirements, the transportation industry sees large poten- 


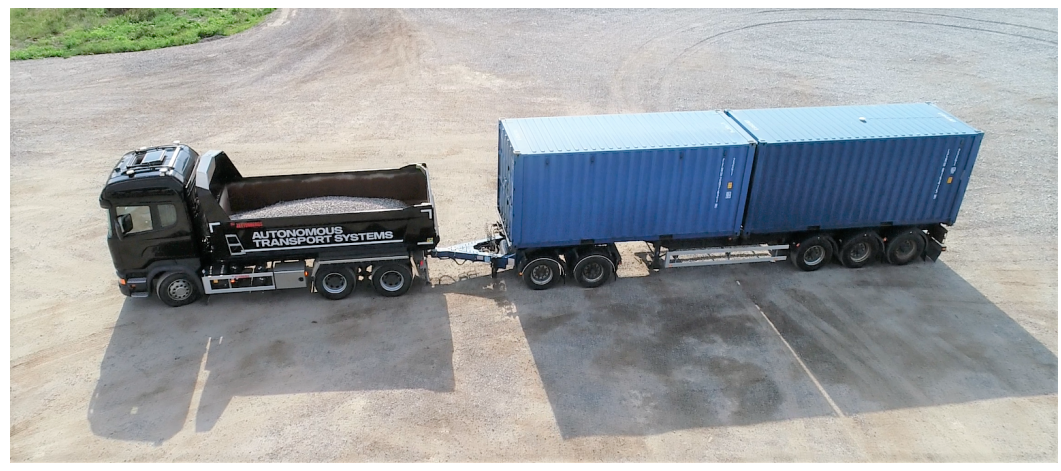

Figure 1.1: The test vehicle that is used as research platform. The truck is a modified version of a Scania R580 6x4, and neither the semitrailer or the dolly is equipped with any sensor. In Paper D and Paper E, this vehicle is used for evaluation of proposed motion planning and control solutions.

tial in automation, electrification and intelligent transportation systems, and are therefore also shifting their attention towards developing autonomous solutions.

Apart from environmental and efficiency aspects, safety is another concern. Annually, over 40 million people are injured in road traffic related accidents (World Health Organization, 2015), where most accidents occur due to human errors (European Commission, 2011). These statistics show that a car is by far the most dangerous transportation alternative (Ernst and Young, 2015). Systems to increase safety have been considered for many years in the automotive industry, where anti-lock braking and electronic stability program are examples of ADAS that have been developed for this purpose. Thanks to recent development in sensor technology, e.g., RADAR, LIDAR and camera sensors, more advanced ADAS have been developed and are now standard in many of today's (modern) cars. These systems can detect and handle critical situations much faster than an average driver. Examples of such systems are lane keeping assist, trailer assist, adaptive cruise control and queue assist. These systems are taking more and more control over the vehicle to aid the driver in critical or mentally exhausting situations.

With even more advanced systems, such as parallel parking assist and the Tesla autopilot, fully autonomous vehicles are getting closer and have the potential to revolutionize the transportation sector. Autonomous vehicles are not only predicted to reduce the traffic related accidents, but also to transform today's transportation towards a more service-based system. Sharing autonomous vehicles is predicted to result in a better overall utilization of the available vehicle fleet and contribute to a more sustainable future (Burns, 2013; Thrun, 2010).

Today, autonomous driving in urban areas with pedestrians, cyclists and other moving vehicles is still a hard challenge with many unsolved problems. This together with the legislation changes needed to drive autonomously on public roads make closed areas, such as mines, harbors and loading/offloading sites, perfect areas for initial deployment of self-driving vehicles. Here, expected positive outcomes are increased productivity and safety, reduced emissions, lower wear on the equipment and the possibility to relieve the human from performing complex or 
dangerous tasks. Within these sites, different tractor-trailer vehicles can be used to efficiently transport goods and other material. One such example is the truck with a dolly-steered semitrailer that is depicted in Figure 1.1. This vehicle combination consists of a truck with front-wheel steering, a dolly and a semitrailer. This system will also be referred to as a general 2-trailer with a car-like tractor, where the word general refers to the non-zero off-axle hitch connection between the truck and the dolly Altafini et al. (2001).

In comparison to cars and trucks without any trailer, tractor-trailer vehicles are larger and have unstable dynamics while reversing. In particular, the dollysteered semitrailer case is extra challenging. These properties increase the difficulty of designing efficient motion planning and feedback control frameworks for such systems, e.g., for autonomous reversing.

Despite the large amount of research efforts focusing on cars (Paden et al., 2016), existing work that explicitly targets motion planning and feedback control problems for general tractor-trailer vehicles is rather limited (Altafini et al., 2001; Kati et al., 2019b; Michałek, 2014; Pradalier and Usher, 2008; Rimmer and Cebon, 2017). Especially, prior to this work, no complete motion planning and control framework has been presented for these types of systems. Furthermore, before this work, neither has any complete such framework been successfully demonstrated in real-world experiments on a full-scale general 2-trailer with a car-like tractor.

Before presenting the contributions of this thesis, a brief overview of a simplified architecture for a self-driving vehicle is first presented.

\subsection{System architecture}

A self-driving vehicle is a complex system that is composed of multiple subsystems, where each subsystem is designed to solve separate tasks. These subsystems communicate by transmitting and retrieving information between each other using a well-defined interface. An overview of a simplified system architecture for a selfdriving vehicle is depicted in Figure 1.2. This work is focusing on the subsystems that are colored in blue, i.e., the motion planner and the feedback controller.

First, a self-driving vehicle needs a mission or a task to perform. In industrial applications, a mission is in many cases defined in a complex way, where multiple vehicles need to cooperate to solve the task. A mission planner or task planner divides this problem into smaller subproblems and delegates them to suitable vehicles based on their capabilities. An example of a mission can be to load a tractor-trailer vehicle with rocks. A solution would be to instruct the tractortrailer vehicle to go to a suitable loading area and the excavator to pick up rocks and load the trailer until it is fully loaded. These subproblems can be further divided into even smaller subproblems. In this thesis, the focus is on solving one subproblem which is of great importance: how to control a tractor-trailer vehicle such that it can automatically move to a desired location without a clearly defined driving path. In such scenarios, the vehicle is said to operate in an unstructured environment and a motion planner is here required to compute how the vehicle should move to the desired location, e.g., a loading bay or a parking spot. 


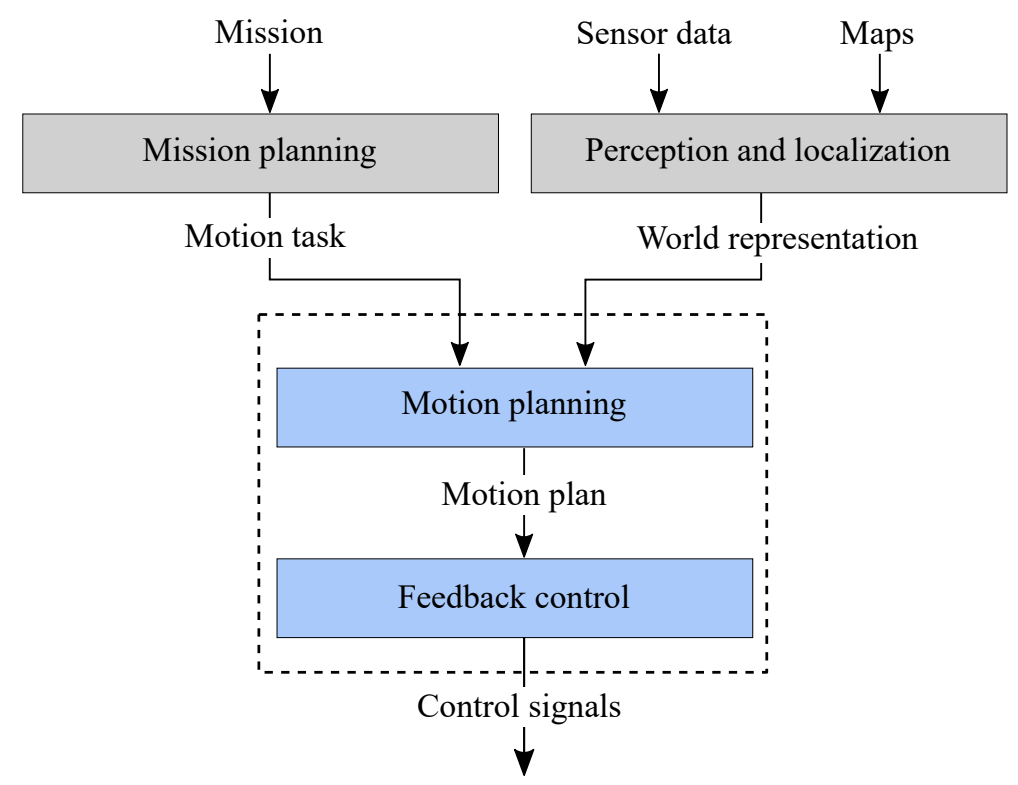

Figure 1.2: An overview of a simplified system architecture for a self-driving vehicle where the blue subsystems are considered in this thesis. Inspired and adapted from (Evestedt, 2016; Lima, 2018).

Before a maneuver can be planned and executed, the system needs to obtain a representation of the surrounding environment and understand where the vehicle is located in the world. These tasks are taken care of by the perception and localization layers, which use measurements from the vehicle's onboard sensors to construct this valuable information. A typical sensor platform on a self-driving vehicle is composed of multiple different sensors, such as global navigation satellite system (GNSS) receivers, e.g. global positioning system (GPS) receivers, inertial measurement units (IMUs), RADAR sensors, LIDAR sensors and cameras. The localization layer combines the sensor information with offline data, such as stored maps, to estimate the vehicle's position, orientation and other important vehicle states (Skog and Händel, 2009). The perception layer makes use of the same sensor information to detect, classify and track different objects, such as people and other vehicles (Granström et al., 2019). Together, these layers provide a compressed representation of the surrounding environment in which motion planning and feedback control is performed.

The motion planner's objective is to compute a motion plan from the vehicle's current state to the desired goal state specified by the mission planner or a human operator. The motion plan is a nominal trajectory or path that, most often, satisfies a mathematical model of the vehicle and is computed such that the vehicle is predicted not to collide with any surrounding obstacle.

To guarantee safe execution of the motion plan, the feedback controller is designed to stabilize the vehicle around the nominal trajectory or path despite that disturbances are acting on the vehicle. 


\subsection{Main contributions}

The main contributions of this thesis are within the area of motion planning and feedback control for tractor-trailer vehicles. The contributions include development of motion planning and feedback control frameworks, some design approaches for guaranteeing closed-loop stability, and experimental validation of the proposed frameworks through simulations, lab and field experiments. State estimation of a full-scale general 2-trailer with a car-like tractor is also considered for the case when neither the semitrailer nor the dolly is equipped with any sensor. Many of the developed solutions are also experimentally validated on either a physical small-scale or a full-scale test vehicle. In summary, the contributions of this thesis are:

- Development of a cascade controller for a reversing general 2-trailer system and the use of this controller within a closed-loop rapidly-exploring random tree (CL-RRT) framework (Paper A). To the authors' knowledge, this was the first sampling-based motion planner for this type of system.

- Derivation of a path-following error model for a general 2-trailer system for which a linear quadratic (LQ) path-following controller is proposed as well as a method for analyzing stability of the closed-loop system, where the resulting constraints on the motion plan can be enforced on a motion planner to guarantee safe path execution (Paper B).

- Modeling of the closed-loop system (a lattice-based planner, a state-feedback controller and a controlled vehicle) as a hybrid system and the development of a tailored controller synthesis and stability analysis tool to a priori guarantee stability (Paper C).

- Development of a path planning and path-following control framework for a general 2-trailer with a car-like tractor including a lattice-based planner, a path-following controller and an observer that only utilizes information from sensors that are mounted on the tractor (Paper D). To the authors' knowledge, this was the first planning and control framework for a full-scale tractor-trailer vehicle that has been successfully demonstrated in practice.

- Development, implementation and experimental evaluation of a model predictive path-following control approach for a general 2-trailer with a car-like tractor in which an advanced sensor's sensing limitations are modeled and incorporated as explicit constraints in the controller (Paper E).

- Generalization of the lattice-based planner in Paper D to also include multisteered articulated vehicles and to propose a post-optimization step that enables the framework to compute locally optimal trajectories that start exactly at the vehicle's initial state and reaches the goal state exactly (Paper F).

- Generalization of the path-following error model in Paper B and parts of the model predictive path-following control approach in Paper E to also include multi-steered articulated vehicles (Paper G). 


\subsection{Thesis outline and contributions}

This thesis is divided into two parts. The first part contains some background material regarding nonholonomic systems as well as popular motion planning and feedback control techniques for such systems. The second part of this thesis is a collection of scientific contributions including six accepted papers and one submitted paper which is an extension of an accepted conference paper. A brief summary of each paper is given below.

\section{Paper A: Motion planning for a reversing general 2-trailer configuration using Closed-Loop RRT}

N. Evestedt, O. Ljungqvist, and D. Axehill. Motion planning for a reversing general 2-trailer configuration using Closed-Loop RRT. In Proceedings of the 2016 IEEE/RSJ International Conference on Intelligent Robots and Systems, pages 3690-3697, 2016b.

Summary: This paper presents a probabilistic motion planning framework based on closed-loop rapidly-exploring random tree (CL-RRT) for a general 2trailer with a car-like tractor. The path-following controller developed in Evestedt et al. (2016a); Ljungqvist (2015) is used to enable efficient closed-loop simulations of the system within the CL-RRT framework presented in Evestedt et al. (2015). The framework is evaluated in a set of simulations in different kinds of environments and in a lab environment with a small-scale test platform. The results show that the planner has a high success rate in finding motion plans in complex and constrained environments.

Background and contribution: The idea of performing closed-loop simulations of a general 2-trailer with a car-like tractor within a CL-RRT framework was initiated from discussions between the Niclas Evestedt and Daniel Axehill. The concept was then developed by the author of this thesis during his Master's thesis project (Ljungqvist, 2015) where Niclas acted as supervisor, and Daniel as examiner and supervisor. In an early stage, the author of this thesis and Niclas initiated a tight collaboration where the underlying CL-RRT platform was developed by Niclas (Evestedt et al., 2015) and the development as well as the integration of the stabilizing controller was performed by the author of this thesis. The experimental platform development and data collections were accomplished jointly between the author of this thesis and Niclas. The majority of the writing was done by Niclas and Daniel acted as supervisor and reviewed the manuscript.

\section{Paper B: Path-following control for a reversing general 2-trailer system}

O. Ljungqvist, D. Axehill, and A. Helmersson. Path following control for a reversing general 2-trailer system. In Proceedings of the 55th IEEE Conference on Decision and Control, pages 2455-2461, 2016.

Summary: A path-following controller for a reversing general 2-trailer with a car-like tractor is proposed for the case when the motion plan is kinematically 
feasible to follow exactly. It is done by first deriving a path-following error model in which the vehicle's path-following error states are described in terms of deviation from the nominal path. A stabilizing LQ controller with feedforward action is then designed. Given that the motion planner computes motion plans for a specified set of possible motions, the origin of the closed-loop system is shown to be an exponentially stable equilibrium point. The theoretical results are verified through simulations of the closed-loop system around an eight-shaped path.

Background and contribution: The idea to this work was initiated through discussion between the author of this thesis, Daniel Axehill and Anders Helmersson. The modeling of the vehicle in the Frenet-Serret frame was performed by the author of this thesis as well as the theoretical derivations, implementations, numerical calculations and the written manuscript. Daniel and Anders acted as supervisors and reviewed the manuscript.

\section{Paper C: On stability for state-lattice trajectory tracking control}

O. Ljungqvist, D. Axehill, and J. Löfberg. On stability for state-lattice trajectory tracking control. In Proceedings of the 2018 American Control Conference, 2018.

Summary: This paper presents a systematic framework for analyzing stability of the closed-loop system consisting of a controlled vehicle and a feedback controller executing a motion plan computed by a lattice-based planner. When this motion planner is considered, it is shown that the closed-loop system can be modeled as a nonlinear hybrid system. Based on this, we propose a novel method for analyzing the behavior of the tracking error, how to design the low-level controller and how to potentially impose constraints on the motion planner, in order to guarantee that the tracking error is bounded and decays towards zero. The proposed method is applied on a tractor-trailer system and the results are verified in simulations.

Background and contribution: The idea to this work evolved after discussion between the author of this thesis and Daniel Axehill. Johan Löfberg was involved in some of the discussions and in particular during the development of Proposition 1. The author of this thesis contributed with the majority of the work including theoretical derivations and the written manuscript. Daniel contributed throughout the process and acted as supervisor and reviewed the manuscript.

\section{Paper D: A path planning and path-following control framework for a general 2-trailer with a car-like tractor}

O. Ljungqvist, N. Evestedt, D. Axehill, M. Cirillo, and H. Pettersson. A path planning and path-following control framework for a general 2-trailer with a car-like tractor. Journal of Field Robotics, 36(8):1345$1377,2019$. 
Summary: This paper presents a complete motion planning and control framework for a general 2-trailer with a car-like tractor that can be used to automatically plan and execute difficult parking and obstacle avoidance maneuvers by combining forward and backward motion. A lattice-based motion planner is utilized to efficiently compute kinematically feasible and collision-free motion plans. To execute the motion plan, a path-following controller is developed that stabilizes the lateral and angular path-following error states of the vehicle. Moreover, a nonlinear observer for state estimation is developed which only utilizes information from sensors that are mounted on the tractor, making the system independent of any additional sensor mounted on the semitrailer. The proposed planning and control framework is implemented on a full-scale test vehicle and a series of field experiments are presented.

Background and contribution: This work summarizes a long project where the path planner from Ljungqvist et al. (2017) and the path-following controller from Paper $\mathrm{C}$ were implemented on a full-scale test platform. Many extensions from those works have been made to cope with the full-scale test platform and have mainly been performed by the author of this thesis together with Niclas Evestedt. Niclas started the implementation of the nonlinear observer before leaving academia. The author of this thesis took over and made many improvements to make the nonlinear observer work robustly for all relevant scenarios. The field experiments and the tuning of all modules were made by the author of this thesis together with Henrik Pettersson. The author of this thesis contributed with the majority of the work including theoretical derivations, numerical calculations, field experiments and the written manuscript. Niclas, Daniel Axehill and Marcello Cirillo contributed throughout the process and reviewed the manuscript.

\section{Paper E: Estimation-aware model predictive path-following control for a general 2-trailer with a car-like tractor}

O. Ljungqvist, D. Axehill, H. Pettersson, and J. Löfberg. Estimationaware model predictive path-following control for a general 2-trailer with a car-like tractor. Submitted to IEEE Transactions on Robotics. Preprint: https://arxiv.org/abs/2002.10291, 2020b.

Summary: This paper presents a model predictive path-following control approach for a general 2-trailer with a car-like tractor. It is inspired by the work in Paper D where an advanced sensor with a limited field of view is placed in the rear of the tractor to solve the joint-angle estimation problem. This implies that the proposed estimation solution introduces restrictions on the joint-angle configurations that can be estimated with high accuracy. To model and explicitly consider these constraints in the controller, a model predictive path-following control approach is proposed. Two approaches with different computation complexity and performance are presented. In simulations and field experiments, the performance of the proposed path-following control approach is compared with the proposed control strategy in Paper D where the joint-angle constraints are neglected. 
Background and contribution: The idea to this work initiated after discussions between the author of this thesis and Daniel Axehill. A preliminary version of the paper was presented in Ljungqvist et al. (2020a). Johan Löfberg assisted in the development of the mixed-integer programming formulation as well as with some implementation details in YALMIP (Löfberg, 2004). The implementation and field experiments were carried out by the author of this thesis together with Henrik Pettersson. The author of this thesis contributed with the majority of the work including theoretical derivations, numerical calculations, field experiments and the written manuscript. Daniel and Johan reviewed the manuscript.

\section{Paper F: Optimization-based motion planning for multi-steered articulated vehicles}

O. Ljungqvist, K. Bergman, and D. Axehill. Optimization-based motion planning for multi-steered articulated vehicles. Accepted for publication in Proceedings of the 21th IFAC World Congress. Preprint: https://arxiv.org/abs/1912.06264, 2020c.

Summary: This paper presents an optimization-based trajectory planner targeting low-speed maneuvers in unstructured environments for multi-steered $\mathrm{N}$ trailer (MSNT) vehicles, which are composed of a car-like tractor and an arbitrary number of $N$ interconnected trailers with fixed or steerable wheels. The proposed trajectory planner uses a lattice-based planner in a first step to compute a resolution optimal solution to a discretized version of the trajectory planning problem. The output from the lattice-based planner is then used in a second step to warmstart an optimal control problem solver, which enables the framework to compute locally optimal trajectories that start at the vehicle's initial state and reaches the goal state exactly. The performance of the trajectory planner is evaluated in a set of parking scenarios for an MS3T vehicle with a car-like tractor where the last trailer is steerable.

Background and contribution: The idea for this work was initiated after discussion between the author of this thesis, Kristoffer Bergman and Daniel Axehill. Throughout the process, the author of this thesis and Kristoffer maintained a tight collaboration. The proposed trajectory planner is based on the work in Bergman et al. (2019a,b) and is here tailored for MSNT vehicles. The implementation was accomplished jointly between the author of this thesis and Kristoffer. The author of this thesis contributed with all derivations, calculations and the written manuscript. Kristoffer and Daniel reviewed the manuscript.

\section{Paper G: A predictive path-following controller for multi-steered articulated vehicles}

O. Ljungqvist and D. Axehill. A predictive path-following controller for multi-steered articulated vehicles. Accepted for publication in Proceedings of the 21th IFAC World Congress. Preprint: https: //arxiv . org/abs/1912.06259, 2020. 
Summary: This paper presents a model predictive path-following control approach for automatic low-speed maneuvering of MSNT vehicles. The proposed path-following controller is tailored to follow nominal paths that contain full state and control-input information, and is designed to satisfy various constraints on the vehicle states as well as saturations and rate limitations on the tractor's curvature and the trailer steering angles. The performance of the proposed model predictive path-following controller is evaluated in a set of simulations for an MS2T vehicle with a car-like tractor where the last trailer is steerable.

Background and contribution: The ideas for this work were mainly initiated by the author of this thesis. The work generalizes and combines parts of the work in Paper B, Paper D and Paper E to include MSNT vehicles. The author of this thesis contributed with the majority of the work including theoretical derivations, implementation, numerical calculations and the written manuscript. Daniel Axehill acted as supervisor and reviewed the manuscript.

\subsection{Other publications}

The following additional publications have been authored or co-authored by the author of this thesis:

N. Evestedt, O. Ljungqvist, and D. Axehill. Path tracking and stabilization for a reversing general 2-trailer configuration using a cascaded control approach. In Proceedings of the 2016 IEEE Intelligent Vehicles Symposium, pages 1156-1161, 2016a.

O. Ljungqvist, N. Evestedt, M. Cirillo, D. Axehill, and O. Holmer. Lattice-based motion planning for a general 2-trailer system. In Proceedings of the 2017 IEEE Intelligent Vehicles Symposium, pages 24552461, 2017.

G. Ling, K. Lindsten, O. Ljungqvist, J. Löfberg, C. Norén, and C. A. Larsson. Fuel-efficient model predictive control for heavy duty vehicle platooning using neural networks. In Proceedings of the 2018 Annual American Control Conference, pages 3994-4001, 2018.

O. Andersson, O. Ljungqvist, M. Tiger, D. Axehill, and F. Heintz. Receding-horizon lattice-based motion planning with dynamic obstacle avoidance. In Proceedings of the 57th IEEE Conference on Decision and Control, pages 4467-4474, $2018 \mathrm{~b}$.

O. Ljungqvist. On motion planning and control for truck and trailer systems. Licentiate's thesis, Linköping University, 2019.

K. Bergman, O. Ljungqvist, and D. Axehill. Improved optimization of motion primitives for motion planning in state lattices. In Proceedings of the 2019 IEEE Intelligent Vehicles Symposium, 2019b. 
K. Bergman, O. Ljungqvist, and D. Axehill. Improved path planning by tightly combining lattice-based path planning and optimal control. Accepted for publication in IEEE Transactions on Intelligent Vehicles. Preprint: https://arxiv.org/abs/1903.07900, 2019a.

R. Oliveira, O. Ljungqvist, P. F. Lima, and B. Wahlberg. Optimizationbased on-road path planning for articulated vehicles. Accepted for publication in Proceedings of the 21th IFAC World Congress. Preprint: https://arxiv.org/abs/2001.06827, 2020a.

O. Ljungqvist, D. Axehill, and H. Pettersson. On sensing-aware model predictive path-following control for a reversing general 2-trailer with a car-like tractor. Accepted for publication in Proceedings of the 2020 IEEE International Conference on Robotics and Automation. Preprint: https://arxiv.org/abs/2002.06874, 2020a.

K. Bergman, O. Ljungqvist, T. Glad, and D. Axehill. An optimizationbased receding horizon trajectory planning algorithm. Accepted for publication in Proceedings of the 21th IFAC World Congress. Preprint: https://arxiv.org/abs/1912.05259, 2020a.

R. Oliveira, O. Ljungqvist, P. F. Lima, and B. Wahlberg. A geometric approach to on-road motion planning for long and multi-body heavyduty vehicles. Accepted for publication in Proceedings of the 31st IEEE Intelligent Vehicles Symposium, 2020b.

K. Bergman, O. Ljungqvist, J. Linder, and D. Axehill. An optimizationbased motion planner for autonomous maneuvering of marine vessels in complex environments. Submitted to the 59th IEEE Conference on Decision and Control, 2020b. 



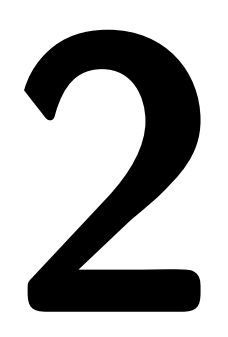

\section{Modeling of wheeled vehicles}

This chapter is intended to present some models that are commonly used for motion planning and feedback control of wheeled vehicles during low-speed maneuvers. This chapter starts with a brief introduction to different modeling techniques. In Section 2.2, a short introduction to nonholonomic systems is presented. In Section 2.3 and Section 2.4, the kinematic bicycle model and the general N-trailer are presented, respectively.

\subsection{Introduction}

Modeling of wheeled vehicles has a long history and depending on the application, different modeling techniques are used (LaValle, 2006; Rajamani, 2011). Roughly speaking, a vehicle model is said to be dynamic if it is derived based on force balances, or kinematic if it is derived based on velocity constraints. These velocity constraints will be further referred to as nonholonomic constraints. For wheeled vehicles, these constraints naturally arise if the wheels of the vehicle are assumed to be rolling without slipping. During low-speed maneuvers on dry road-surface conditions, a kinematic model is often sufficient to describe the behavior of a wheeled vehicle (LaValle, 2006; Paden et al., 2016; Spong et al., 2006). On the contrary, during aggressive high-speed maneuvers, the dynamical effects become more prominent and a dynamic model is required to capture these effects (Anistratov et al., 2018; Berntorp et al., 2014; Falcone et al., 2007; Fors et al., 2019; Kati et al., 2019a,b). In this chapter, the basic tools for modeling the kinematic properties of a wheeled vehicle are presented. Here, only a brief introduction is provided and the reader is referred to, e.g., LaValle (2006); Spong et al. (2006) for a more detailed coverage. A major reason why more advanced vehicle models with higher fidelity are usually not used for planning is that advanced models imply a high-dimensional state-space. Since most practical applications require 
efficient motion planning modules, kinematic models with a lower state dimension are commonly used (Paden et al., 2016). However, a more advanced vehicle model could preferably be used for simulation purposes (Lima, 2018; Rajamani, 2011) or in the design of safety modules to handle evasive maneuvering (Fors et al., 2019; Svensson et al., 2019).

\subsection{Nonholonomic systems}

A kinematic model of a wheeled vehicle is derived based on the assumption that the wheels of the vehicle are rolling without slipping. This implies that there exist velocity directions in which the vehicle cannot move. Systems subject to such velocity constraints are referred to as nonholonomic systems.

Let $q \in \mathbb{R}^{n}$ denote a configuration of the vehicle, i.e., an $n$-dimensional vector of generalized coordinates. The configuration space $\mathcal{C} \subseteq \mathbb{R}^{n}$ is defined as the manifold of all possible vehicle configurations and is assumed to be a smooth manifold (LaValle, 2006). For modeling of wheeled vehicles, we are particularly interested of constraints in the following form

$$
g(q, \dot{q})=0
$$

where $\dot{q}=\mathrm{d} q / \mathrm{d} t$. These are constraints on the velocities of the system, e.g., a car cannot move sideways. In some cases, velocity constraints can be explicitly integrable, giving rise to constraints in the form

$$
h(q)=0,
$$

which are algebraic constraints in the configuration of the vehicle. Such constraints are said to be holonomic and the motion of the vehicle is thus restricted to a level surface of $h$. If a velocity constraint is not explicitly integrable the constraint is said to be nonholonomic. There exist different holonomic and nonholonomic constraints that naturally arise due to physical limitations on the system. Two common examples are:

$$
\begin{array}{ll}
h(q) \leq 0: & \text { configuration inequality constraint, } \\
h(\dot{q}) \leq 0: & \text { velocity inequality constraint. }
\end{array}
$$

These are inequality constraints on the configuration $q$ and the velocity $\dot{q}$ of the system and two examples of such constraints are limited steering angle and steering angle rate for a car. In the remainder of this chapter we focus on the special class of nonholonomic systems that have linear velocity constraints

$$
\omega_{i}(q) \dot{q}=0, \quad i=1, \ldots, k<n,
$$

where $\omega_{i}(q) \in \mathbb{R}^{1 \times n}$. These constraints are called Pfaffian constraints and it is assumed that $\omega_{i}, i=1, \ldots, k$ are smooth functions and linearly independent for all $q \in \mathcal{C}$. An interpretation of the constraints in (2.3) is that the vector fields of the system have to be orthogonal to each $\omega_{i}(q)$. The linear velocity constraints in $(2.3)$ can be represented on matrix form as $\omega(q) \dot{q}=0$, where $\omega(q) \in \mathbb{R}^{k \times n}$. 
Instead of expressing which velocity directions the vehicle cannot move, it is more convenient to express which directions it can, i.e., transform the constraints in (2.3) to explicit form $\dot{q}=f(q, u)$ (LaValle, 2006). Let $m=n-k>0$ and choose $g_{1}, \ldots, g_{m}$ as a basis of right null-space of $\omega(q)$. By assigning each $g_{j} \in \mathbb{R}^{n}$ with a control signal $u_{j}$, a control-affine driftless system is obtained

$$
\dot{q}=\sum_{j=1}^{m} g_{j}(q) u_{j},
$$

where each $u_{j}, j=1, \ldots, m$, typically corresponds to a physical actuator. A common property for nonholonomic systems is that they are underactuated, which means that the number of control signals $m$ is less than the dimension of the configuration-space $n$. Generally, a nonholonomic system can be modeled as a nonlinear system

$$
\dot{x}=f(x, u),
$$

where the state vector $x$ is typically chosen to coincide with the configuration $x=q$ or additional properties are also modeled, e.g., $x=\left[\begin{array}{ll}q^{\top} & \dot{q}^{\top}\end{array}\right]^{\top}$ when dynamics are also considered (LaValle, 2006). However, the degree of freedom $m$ for the system will not change. In the remainder of this chapter, models of nonholonomic systems that are of particular importance for this thesis are derived.

\subsection{The kinematic bicycle model}

A vehicle model that is widely used in the motion planning and control literature is the kinematic bicycle model (LaValle, 2006; Paden et al., 2016). During lowspeed maneuvers, cars and trucks (Lima, 2018; Paden et al., 2016; Werling et al., 2010) have been shown to be sufficiently well described by this relatively simple model. The model is derived based on the assumption that the wheels of the vehicle are rolling without slipping. The vehicle is also assumed to operate on a flat surface and assumed to be front-wheel steered with perfect Ackerman steering (see Figure 2.1). Briefly, the Ackerman steering geometry makes the absolute value of the turning radius for inner front wheel smaller than the absolute value of the outer front wheel's turning radius. As illustrated in Figure 2.1, this implies that there exists a resulting front-wheel steering angle $\alpha$ that can replace the inner and outer steering angles.

The wheelbase of the vehicle is denoted by $L_{1}$ and the longitudinal velocity of its rear axle is denoted by $v_{1}$. The configuration of the vehicle is defined as $q=\left[\begin{array}{llll}x_{1} & y_{1} & \theta_{1} & \alpha\end{array}\right]^{\top}$, where $\left(x_{1}, y_{1}\right)$ is the position of the center of the vehicle's rear axle and $\theta_{1}$ is the vehicle's orientation. Under no-slip assumptions, the component of the velocity vector that is orthogonal to the rear wheels is zero which leads to the following linear velocity constraint:

$$
\underbrace{\left[\begin{array}{cccc}
-\sin \theta_{1} & \cos \theta_{1} & 0 & 0
\end{array}\right]}_{\triangleq \omega_{1}(q)} \dot{q}=0 .
$$




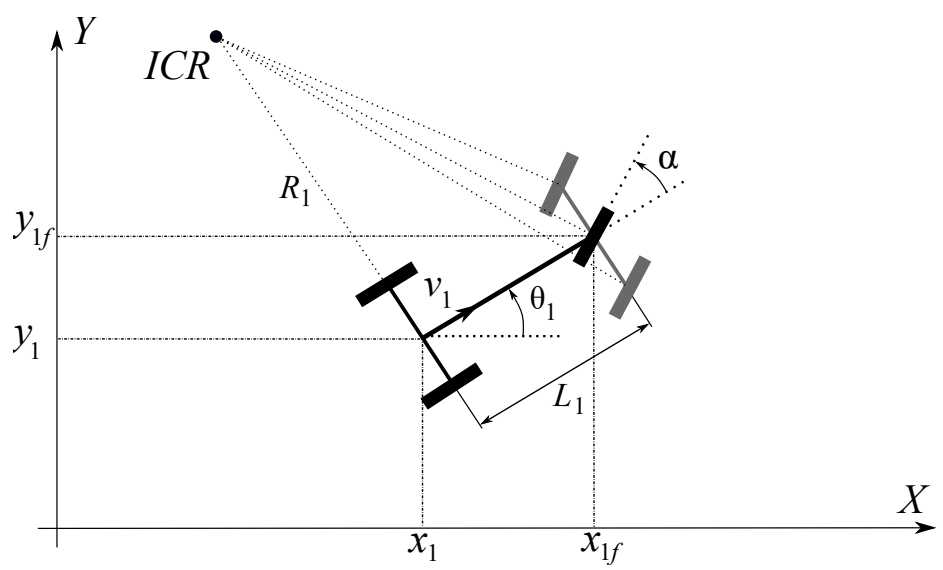

Figure 2.1: A schematic illustration of the kinematic bicycle model.

Repeating this argument for the front wheel implies that the following nonholonomic constraint also has to be satisfied

$$
-\dot{x}_{1 \mathrm{f}} \sin \left(\theta_{1}+\alpha\right)+\dot{y}_{1 \mathrm{f}} \cos \left(\theta_{1}+\alpha\right)=0 .
$$

The position of the center of the front axle $\left(x_{1 \mathrm{f}}, y_{1 \mathrm{f}}\right)$ can be expressed as a function of $q$ as

$$
\begin{aligned}
& x_{1 \mathrm{f}}=x_{1}+L_{1} \cos \theta_{1}, \\
& y_{1 \mathrm{f}}=y_{1}+L_{1} \sin \theta_{1} .
\end{aligned}
$$

By differentiating (2.8) with respect to time and inserting the result in (2.7), the second nonholonomic constraint that has to hold is

$$
\underbrace{\left[\begin{array}{llll}
-\sin \left(\theta_{1}+\alpha\right) & \cos \left(\theta_{1}+\alpha\right) & L_{1} \cos \alpha & 0
\end{array}\right]}_{\triangleq \omega_{2}(q)} \dot{q}=0 .
$$

Provided that the steering angle satisfies $|\alpha|<\pi / 2$, the row vectors $\omega_{1}(q)$ and $\omega_{2}(q)$ are linearly independent and a basis of the right null space to $\omega(q)=\left[\begin{array}{ll}\omega_{1}^{\top} \omega_{2}^{\top}\end{array}\right]^{\top}$ is given by

$$
g_{1}=\left[\begin{array}{c}
\cos \theta_{1} \\
\sin \theta_{1} \\
\frac{\tan \alpha}{L_{1}} \\
0
\end{array}\right], \quad g_{2}=\left[\begin{array}{l}
0 \\
0 \\
0 \\
1
\end{array}\right]
$$

That is, the kinematic bicycle model can be written as a control-affine driftless system (2.4) with $m=2$, and one possible combination of control signals is the longitudinal velocity $u_{1}=v_{1}$ and the steering angle rate $u_{2}=\dot{\alpha}$.

In trajectory-tracking and path-following control applications during low-speed maneuvers, the dynamics in the steering angle is sometimes neglected and $\alpha$ is 
assumed to be directly controlled (Paden et al., 2016). For this case, the state vector is defined as $x=\left[\begin{array}{lll}x_{1} & y_{1} & \theta_{1}\end{array}\right]^{\top}$ and the control-input vector as $u=\left[\begin{array}{ll}v_{1} & \alpha\end{array}\right]^{\top}$. The resulting simplified version of the kinematic bicycle model is

$$
\dot{x}=v_{1}\left[\begin{array}{c}
\cos \theta_{1} \\
\sin \theta_{1} \\
\frac{\tan \alpha}{L_{1}}
\end{array}\right] .
$$

Here, the steering angle $\alpha$ can be substituted with the car-like vehicle's curvature $\kappa$ which is defined as

$$
\kappa=\frac{1}{R_{1}}=\frac{\mathrm{d} \theta_{1}}{\mathrm{~d} s_{x}}=\frac{\tan \alpha}{L_{1}},
$$

where $R_{1}$ is the turning radius of the vehicle and $s_{x}(t)=\int_{0}^{t}\left|v_{1}(\tau)\right| d \tau$ is the distance traveled by the rear axle of the vehicle.

Dubins' version of the bicycle model (2.11) is obtained by restricting the control inputs as $v_{1} \in\{0,1\}$ and $\alpha \in\left\{-\alpha_{\max }, 0, \alpha_{\max }\right\}$, which means that the vehicle is only allowed to stand still or move straight, maximum left or maximum right at constant forward speed $v_{1}=1$ (Dubins, 1957). Reeds-Shepp's version is obtained by allowing the vehicle to also travel in backward motion $v_{1} \in\{-1,0,1\}$ (Reeds and Shepp, 1990).

Since $v_{1}$ enters bilinearly into the model in (2.11), a method known as timescaling (Houska et al., 2011b) can be applied to eliminate the longitudinal speed $\left|v_{1}\right|$ from the model. Since $\dot{s}_{x}=\left|v_{1}\right|$ holds, by applying the chain-rule $\frac{\mathrm{d} x}{\mathrm{~d} t}=\frac{\mathrm{d} x}{\mathrm{~d} s_{x}}\left|v_{1}\right|$ the model in (2.11) can be written on spatial form as

$$
\frac{\mathrm{d} x}{\mathrm{~d} s_{x}}=\bar{v}_{1}\left[\begin{array}{c}
\cos \theta_{1} \\
\sin \theta_{1} \\
\kappa
\end{array}\right],
$$

where $\bar{v}_{1}=\operatorname{sign}\left(v_{1}\right)=\{-1,1\}$, i.e., $\bar{v}_{1}=1$ denotes forward motion and $\bar{v}_{1}=-1$ denotes backward motion. This result is frequently used as motivation for decoupling the motion planning problem into path planning and velocity planning when low-speed maneuvers are considered. It is also a strong motivation for decoupling the feedback control problem into lateral and longitudinal control.

\subsection{The general $\mathrm{N}$-trailer with a car-like tractor}

A family of systems that is of special interest for this thesis is the so-called N-trailer vehicles (Altafini, 2001; Michałek, 2013b; Sørdalen, 1993). These systems consist of $N+1$ vehicle segments including a leading car-like tractor that is connected to $N$ passive trailers with on-axle or signed off-axle hitch connections. Each vehicle segment is characterized by a segment length $L_{i}>0$ and a signed hitching offset $M_{i}$ that is positive if the connection is behind the preceding vehicle segment's axle. If the system has mixed hitching types it is called a general N-trailer, and in case of pure on-axle or off-axle hitching, it is referred to as a standard N-trailer or a non-standard N-trailer, respectively (Michałek, 2013b). 


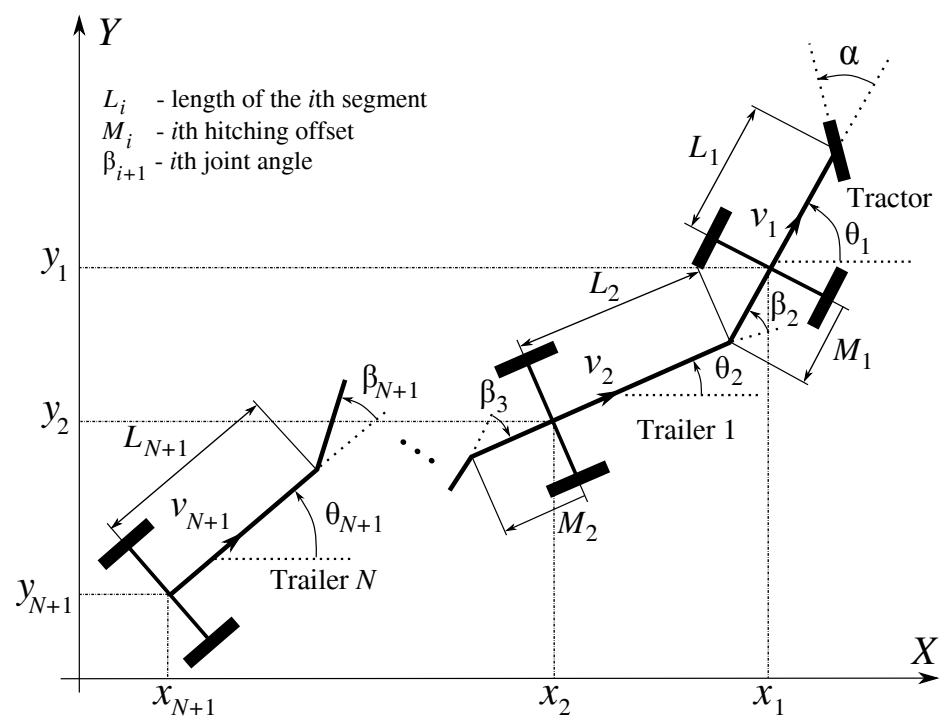

Figure 2.2: A schematic description of the general $N$-trailer with a car-like tractor. The system consists of a leading car-like tractor that is connected to $N$ passive trailers with a mixture of on-axle and off-axle hitch connections.

The control inputs to an N-trailer vehicle with a car-like tractor are the tractor's longitudinal velocity of its rear axle $v_{1}$ and its front-wheel steering angle $\alpha$ (or the tractor's curvature $\left.\kappa=\tan \alpha / L_{1}\right)$. Similar to the kinematic bicycle model, the kinematic model for an N-trailer vehicle is also derived based on holonomic and nonholonomic constraints and a recursive formula is presented in Altafini (2001). The model is derived based on the assumptions that the wheels are rolling without slipping and that the vehicle is operating on a flat surface. A schematic description of the system is provided in Figure 2.2 and the state vector consists of $3+N$ variables:

- the global position $\left(x_{l}, y_{l}\right)$ and orientation $\theta_{l}$ of the $l$-th vehicle segment in a fixed coordinate frame

$$
q_{l}=\left[\begin{array}{lll}
x_{l} & y_{l} & \theta_{l}
\end{array}\right]^{\top} \in \mathbb{R}^{2} \times \mathbb{S}, \quad \mathbb{S}=(-\pi, \pi],
$$

- for $i=2, \ldots, N+1$, a number of $N$ joint angles

$$
\beta_{i}=\theta_{i-1}-\theta_{i} \in \mathcal{B}_{i}=\left[-\bar{\beta}_{i}, \bar{\beta}_{i}\right], \quad \bar{\beta}_{i} \in(0, \pi) .
$$

The state vector for the general N-trailer with a car-like tractor is defined as

$$
x=\left[\begin{array}{llll}
q_{l}^{\top} & \beta_{2} & \ldots & \beta_{N+1}
\end{array}\right]^{\top} \in \mathcal{X},
$$

where $\mathcal{X}=\mathbb{R}^{2} \times \mathbb{S} \times \mathcal{B}_{2} \ldots \times \mathcal{B}_{N+1}$. 
The leading car-like tractor is described by a kinematic bicycle model (2.11) and thus, its yaw-rate is

$$
\dot{\theta}_{1}=v_{1} \kappa,
$$

where $\kappa=\tan \alpha / L_{1}$. The recursive formula for the transformation of the angular velocity $\dot{\theta}_{i}$ and the longitudinal velocity $v_{i}$ between any two neighboring vehicle segments are given by (Altafini, 2001; Michałek, 2013b):

$$
\left[\begin{array}{c}
\dot{\theta}_{i} \\
v_{i}
\end{array}\right]=\underbrace{\left[\begin{array}{cc}
-\frac{M_{i-1} \cos \beta_{i}}{L_{i}} & \frac{\sin \beta_{i}}{L_{i}} \\
M_{i-1} \sin \beta_{i} & \cos \beta_{i}
\end{array}\right]}_{\triangleq J_{i}\left(\beta_{i}\right)}\left[\begin{array}{c}
\dot{\theta}_{i-1} \\
v_{i-1}
\end{array}\right], \quad i=2, \ldots, N+1 .
$$

For details regarding the derivation, the reader is referred to, e.g., Altafini (2001); Ljungqvist (2015); Michałek (2013b). The position of the $l$-th vehicle segment evolves according to standard unicycle kinematics

$$
\begin{aligned}
& \dot{x}_{l}=v_{l} \cos \theta_{l}, \\
& \dot{y}_{l}=v_{l} \sin \theta_{l},
\end{aligned}
$$

where its angular rate $\dot{\theta}_{l}$ and longitudinal velocity $v_{l}$ are given by

$$
\left[\begin{array}{l}
\dot{\theta}_{l} \\
v_{l}
\end{array}\right]=\prod_{i=N-l}^{N-2} J_{N-i}\left(\beta_{N-i}\right)\left[\begin{array}{c}
v_{1} \kappa \\
v_{1}
\end{array}\right]=v_{1} \prod_{i=N-l}^{N-2} J_{N-i}\left(\beta_{N-i}\right)\left[\begin{array}{c}
\kappa \\
1
\end{array}\right],
$$

where $\prod_{i=N-l}^{N-2} J_{N-i}\left(\beta_{N-i}\right)=J_{l}\left(\beta_{l}\right) J_{l-1}\left(\beta_{l-1}\right) \cdots J_{2}\left(\beta_{2}\right)$. Note that $(2.20)$ is derived by recursive usage of (2.18) for $l-1$ times together with (2.17). Now, by introducing the vectors $c=\left[\begin{array}{ll}1 & 0\end{array}\right]^{\top}$ and $d=\left[\begin{array}{ll}0 & 1\end{array}\right]^{\top}$, the longitudinal velocity $v_{l}$ and the angular velocity $\dot{\theta}_{l}$ of the $l$-th vehicle segment can be written as

$$
\begin{aligned}
& v_{l}=v_{1} d^{\top} \prod_{i=N-l}^{N-2} J_{N-i}\left(\beta_{N-i}\right)\left[\begin{array}{c}
\kappa \\
1
\end{array}\right] \triangleq v_{1} f_{v_{l}}(x, \kappa), \\
& \dot{\theta}_{l}=v_{1} c^{\top} \prod_{i=N-l}^{N-2} J_{N-i}\left(\beta_{N-i}\right)\left[\begin{array}{c}
\kappa \\
1
\end{array}\right] \triangleq v_{1} f_{\theta_{l}}(x, \kappa),
\end{aligned}
$$

and the model for the position of the $l$-th vehicle segment (2.19) can be written as

$$
\begin{gathered}
\dot{x}_{l}=v_{1} f_{v_{l}}(x, \kappa) \cos \theta_{l}, \\
\dot{y}_{l}=v_{1} f_{v_{l}}(x, \kappa) \sin \theta_{l} .
\end{gathered}
$$

Finally, by combining (2.17)-(2.18), the time derivative of (2.15) yields the kinematics for the joint angles $\dot{\beta}_{i}=\dot{\theta}_{i-1}-\dot{\theta}_{i}$, where

$$
\dot{\beta}_{i}=v_{1} c^{\top}\left(\prod_{j=N-i+1}^{N-2} J_{N-j}\left(\beta_{N-j}\right)-\prod_{j=N-i}^{N-2} J_{N-j}\left(\beta_{N-j}\right)\right)\left[\begin{array}{c}
\kappa \\
1
\end{array}\right] \triangleq v_{1} f_{\beta_{i}}(x, \kappa),
$$


for $i=2, \ldots, N+1$. To conclude, the model for the general N-trailer with a car-like tractor is given in (2.21b)-(2.23) and can compactly be written as

$$
\dot{x}=v_{1} f(x, \kappa) .
$$

The direction of motion is essential for the stability of the system (2.24), where the joint-angle kinematics (2.23) are structurally unstable in backward motion $\left(v_{1}<0\right)$, where it risks to fold and enter what is called a jack-knife state (Altafini, 2001). In forward motion $\left(v_{1}>0\right)$, these modes are stable. Moreover, as for the kinematic bicycle model (2.13), since the longitudinal velocity $v_{1}$ enters bilinearly into the model in (2.24), time-scaling (Houska et al., 2011b) can be applied to eliminate the dependence on the longitudinal speed $\left|v_{1}\right|$.

For the special case with only on-axle hitching $\left(M_{i}=0, i=1, \ldots, N\right)$ the model of the general N-trailer coincides with the standard N-trailer (Sørdalen, 1993). The standard N-trailer is differentially flat (Rouchon et al., 1993) and can be converted into chained form where the position of the $N$-th trailer $\left(x_{N+1}, y_{N+1}\right)$ is the socalled flat output (Sørdalen, 1993). This result has lead to that the pose for the axle of the $N$-th trailer is commonly used to represent the global pose of the vehicle. However, if mainly forward motion is considered, by selecting the car-like tractor as guidance segment may drastically simplify, e.g., controller design, as the jointangle kinematics is structurally stable in forward motion and can to some extent be neglected. The general 1-trailer with a car-like tractor is also differentially flat using a certain choice of flat output. However, when $N \geq 2$, the flatness property does not hold for the general N-trailer case (Rouchon et al., 1993).

The model of the general N-trailer is small-time locally controllable (Khalil and Grizzle, 2002), except from some singularities (Altafini, 2001). The practical interpretation of these singularities is that the vehicle segments are arranged such that the car-like tractor can move while some of the trailers remain static.

\section{A general 2-trailer with a car-like tractor}

The chapter is concluded with the derivation of the kinematic model for a general 2-trailer with a car-like tractor that will be extensively used in Part II of this thesis. The vehicle is illustrated in Figure 2.3 and is composed of a leading carlike tractor, an off-axle hitched dolly $\left(M_{1} \neq 0\right)$ and an on-axle hitched semitrailer $\left(M_{2}=0\right)$. Since the vehicle consists of three segments, two joint angles $\beta_{2}$ and $\beta_{3}$ are needed to describe a configuration of the vehicle. Moreover, as both backward and forward motions are considered in this work, the axle of the semitrailer is used to represent the global pose $q_{3}=\left[\begin{array}{lll}x_{3} & y_{3} & \theta_{3}\end{array}\right]^{\top}$ of the vehicle. That is, the state vector (2.16) is defined as $x=\left[\begin{array}{lll}q_{3}^{\top} & \beta_{2} & \beta_{3}\end{array}\right]^{\top}$ and the control inputs are $v_{1}$ and $\kappa$.

Following the derivations in Section 2.4, the matrices $J_{2}\left(\beta_{2}\right)$ and $J_{3}\left(\beta_{3}\right)$ describing the longitudinal and angular velocity transformations between neighboring vehicle segments (2.18) are

$$
J_{2}\left(\beta_{2}\right)=\left[\begin{array}{cc}
-\frac{M_{1} \cos \beta_{2}}{L_{2}} & \frac{\sin \beta_{2}}{L_{2}} \\
M_{1} \sin \beta_{2} & \cos \beta_{2}
\end{array}\right], \quad J_{3}\left(\beta_{3}\right)=\left[\begin{array}{cc}
0 & \frac{\sin \beta_{3}}{L_{3}} \\
0 & \cos \beta_{3}
\end{array}\right],
$$




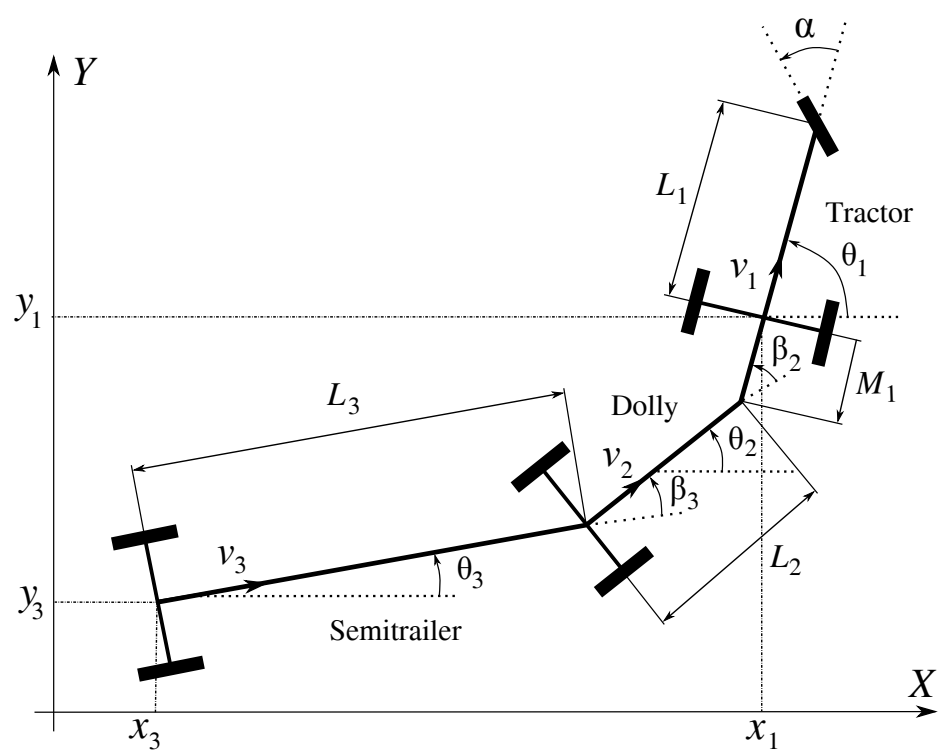

Figure 2.3: An illustration of a general 2-trailer with a car-like tractor.

since $M_{1} \neq 0$ and $M_{2}=0$. Using (2.25) the functions describing the longitudinal and angular velocity transformations from the car-like tractor to the semitrailer (2.21) are

$$
\begin{aligned}
& f_{v_{3}}(x, \kappa)=d^{\top} J_{3}\left(\beta_{3}\right) J_{2}\left(\beta_{2}\right)\left[\begin{array}{l}
\kappa \\
1
\end{array}\right]=\cos \beta_{3}\left(\cos \beta_{2}+M_{1} \sin \beta_{2} \kappa\right), \\
& f_{\theta_{3}}(x, \kappa)=c^{\top} J_{3}\left(\beta_{3}\right) J_{2}\left(\beta_{2}\right)\left[\begin{array}{l}
\kappa \\
1
\end{array}\right]=\frac{\sin \beta_{3}}{L_{3}}\left(\cos \beta_{2}+M_{1} \sin \beta_{2} \kappa\right) .
\end{aligned}
$$

Thus, the model for the pose $q_{3}$ of the semitrailer $(2.21 \mathrm{~b})-(2.22)$ is

$$
\begin{aligned}
& \dot{x}_{3}=v_{1} f_{v_{3}}(x, \kappa) \cos \theta_{3}=v_{1} \cos \beta_{3}\left(\cos \beta_{2}+M_{1} \sin \beta_{2} \kappa\right) \cos \theta_{3}, \\
& \dot{y}_{3}=v_{1} f_{v_{3}}(x, \kappa) \sin \theta_{3}=v_{1} \cos \beta_{3}\left(\cos \beta_{2}+M_{1} \sin \beta_{2} \kappa\right) \sin \theta_{3}, \\
& \dot{\theta}_{3}=v_{1} f_{\theta_{3}}(x, \kappa)=v_{1} \frac{\sin \beta_{3}}{L_{3}}\left(\cos \beta_{2}+M_{1} \sin \beta_{2} \kappa\right) .
\end{aligned}
$$

Finally, using (2.25) in (2.23) the models for the joint-angle kinematics are

$$
\begin{aligned}
\dot{\beta}_{2} & =v_{1} c^{\top}\left[I-J_{2}\left(\beta_{2}\right)\right]\left[\begin{array}{l}
\kappa \\
1
\end{array}\right]=v_{1}\left(\kappa-\frac{\sin \beta_{2}}{L_{2}}+\frac{M_{1}}{L_{2}} \cos \beta_{2} \kappa\right), \\
\dot{\beta}_{3} & =v_{1} c^{\top}\left[J_{2}\left(\beta_{2}\right)-J_{3}\left(\beta_{3}\right) J_{2}\left(\beta_{2}\right)\right]\left[\begin{array}{c}
\kappa \\
1
\end{array}\right] \\
& =v_{1}\left(\frac{1}{L_{2}}\left(\sin \beta_{2}-M_{1} \kappa \cos \beta_{2}\right)-\frac{\sin \beta_{3}}{L_{3}}\left(\cos \beta_{2}+M_{1} \sin \beta_{2} \kappa\right)\right) .
\end{aligned}
$$

The kinematic model of the general 2-trailer with a car-like tractor is given in (2.27)(2.28) and can compactly be written as $\dot{x}=v_{1} f(x, \kappa)$. 



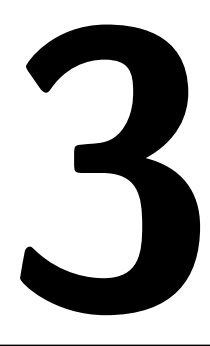

\section{Motion planning for self-driving vehicles}

This chapter presents motion planning techniques for self-driving vehicles. The chapter starts with a short introduction to the motion planning concept. In Section 3.2, the motion planning problem is presented as an optimal control problem (OCP) followed by a discussion of why this OCP is hard to solve by directly applying numerical optimal control. In Section 3.3, the common steps for solving a motion planning problem are explained and in Section 3.4, the basic tools for nonholonomic motion planning are presented. Finally, Section 3.5 contains a definition of a general sampling-based motion planner together with descriptions of some of the popular deterministic and probabilistic versions.

\subsection{Introduction}

The concept of motion planning is and has been an active research topic in different research fields for many decades. However, depending on the field, the meaning of planning differs. In the field of artificial intelligence, planning historically refers to finding a sequence of logical decisions or actions that transforms an initial world state to a desired goal state where system dynamics is often neglected or simplified (Russell and Norvig, 2016). In this thesis, this planning domain will be referred to as mission planning or task planning. For a self-driving vehicle, this type of planner acts as a high-level planner that, e.g., specifies a mission to be executed by the low-level motion planning and control layer.

In this thesis, the motion planning problem is referred to as the problem of computing a feasible trajectory or path that moves the vehicle from its initial state to a desired goal state. The motion plan must satisfy the vehicle's physically imposed constraints as well as constraints imposed by surrounding obstacles while at the same time minimize a specified performance measure. From a control perspective, motion planning is an optimal control problem that in general is hard 
solve due to its non-convex nature. In self-driving vehicle applications, it is also required that a motion plan is computed, and possibly updated, within seconds or even milliseconds. To fulfill these requirements, numerous of different motion planning algorithms have been proposed (Kuwata et al., 2009; LaValle, 1998; Paden et al., 2016; Pivtoraiko et al., 2009). Many of these algorithms originate from the robotics and computer science communities which has been adapted to also take system dynamics into account (LaValle, 2006; Lynch and Park, 2017).

This chapter focuses on motion planning for self-driving vehicles in unstructured environments, e.g., parking lots or other open areas where no clearly defined driver path is available and the vehicle's speed is low. This problem is fundamentally different from motion planning in structured environments, e.g., onroad driving, where information of the road center line is commonly used to enforce structure into the problem. This leads to a problem formulation that has strong similarities to receding horizon control (Batkovic et al., 2019; Lima et al., 2017b; Oliveira et al., 2020a; Paden et al., 2016; Werling et al., 2012; Ziegler and Stiller, 2009).

\subsection{Problem formulation}

The vehicle under consideration is assumed to be modeled as a time-invariant nonlinear system

$$
\dot{x}(t)=f(x(t), u(t)), \quad x\left(t_{I}\right)=x_{I},
$$

where $x(t) \in \mathbb{R}^{n}$ denotes the state vector, $u(t) \in \mathbb{R}^{m}$ the denotes the control inputs and $x_{I}$ is the vehicle's initial state. The vehicle is assumed to have physical limitations on its states $x(t) \in \mathbb{X}$ and control signals $u(t) \in \mathbb{U}$, where $\mathbb{X} \subseteq \mathbb{R}^{n}$ and $\mathbb{U} \subseteq \mathbb{R}^{m}$ are typically convex sets. The vehicle is operating in an environment where static and possibly also moving obstacles exist. The regions which are occupied with obstacles $\mathbb{X}_{\mathrm{obs}}(t)$ are usually divided into static obstacles $\mathbb{X}_{\text {obs }}^{s}$ and dynamic obstacles $\mathbb{X}_{\text {obs }}^{d}(t)$. The free-space where the vehicle is not in collision with any obstacle at time $t$ is defined as $\mathbb{X}_{\text {free }}(t)=\mathbb{X} \backslash \mathbb{X}_{\text {obs }}(t)$. For now, we make the assumption that the set $\mathbb{X}_{\text {free }}(t)$ can be described analytically. Since the free-space $\mathbb{X}_{\text {free }}(t)$ is defined as the complement set of $\mathbb{X}_{\text {obs }}(t)$, it is in general a non-convex set. The objective functional $J$ to be minimized is often composed of different costs, e.g., time duration and/or energy.

The motion planning problem is defined as follows: compute a feasible and collision-free motion plan, i.e., a trajectory $(x(t), u(t)), t \in\left[t_{I}, t_{G}\right]$ that moves the vehicle from its initial state $x_{I}$ to a desired goal state $x_{G}$, while minimizing the objective functional $J$. This problem can be posed as an OCP in the form

$$
\begin{array}{cl}
\underset{u(\cdot), t_{G}}{\operatorname{minimize}} & J=\int_{t_{I}}^{t_{G}} L(t, x(t), u(t)) \mathrm{d} t \\
\text { subject to } & \dot{x}(t)=f(x(t), u(t)), \\
& x\left(t_{I}\right)=x_{I}, \quad x\left(t_{G}\right)=x_{G}, \\
& x(t) \in \mathbb{X}_{\text {free }}(t), \quad u(t) \in \mathbb{U},
\end{array}
$$


where $L>0$ is the so-called cost function. It is assumed that $x_{I} \in \mathbb{X}_{\text {free }}(t)$ and $x_{G} \in \mathbb{X}_{\text {free }}(t)$, for $t \geq t_{I}$ to make the OCP in (3.2) well defined and it is also assumed that there exists a terminal control signal $u_{T}$ such that the vehicle remains at the goal for $t \geq t_{G}$ (LaValle, 2006). The latter assumption is equivalent to that the vehicle ends up in an equilibrium point at the goal, i.e., $f\left(x(t), u_{T}\right)=0$, for $t \geq t_{G}$. This assumption implies that the motion plan can be extended for an arbitrary time duration. The terminal control signal is easy to construct for wheeled vehicles by simply choosing the longitudinal velocity $v_{1, T}=0$ which implies $f\left(x(t), u_{T}\right)=0$. In some applications, the terminal constraint $x\left(t_{G}\right)=x_{G}$ is replaced with a goal region $x\left(t_{G}\right) \in \mathbb{X}_{G}$ (LaValle, 2006). The OCP in (3.2) is in general hard to solve by directly invoking a state-of-the-art numerical optimal control solver (Andersson et al., 2018a; Houska et al., 2011b). Three fundamental reasons for this are:

1. Even if $\mathbb{X}_{\mathrm{obs}}(t)=\emptyset$, the OCP in (3.2) is a non-convex optimization problem because of the nonlinear vehicle model. Thus, for complex systems, a proper warm-start is needed to even find a feasible solution.

2. $\mathbb{X}_{\text {free }}(t)$ is often constructed by fusing information from precomputed maps and onboard sensors on the vehicle and an analytical expression for the obstacle region $\mathbb{X}_{\mathrm{obs}}(t)$ might be difficult to construct in practice.

3. $\mathbb{X}_{\text {free }}(t)$ is often non-convex and the OCP in (3.2) therefore includes a combinatorial problem with possibly different classes of solution trajectories. A proper initial guess is therefore required to find a good locally optimal (or even a feasible) solution to the motion planning problem.

Besides the above mentioned problems, computation efficiency and the absence of real-time guarantees for nonlinear solvers are also strong reasons why standalone numerical optimal control is rarely used in practice. However, numerical optimal control still plays an important role in many motion planning algorithms.

For many nonholonomic systems, the trajectory planning problem in (3.2) can be converted into a path planning planning when dynamic obstacles are neglected. The relationship between a path and a trajectory is defined as follows.

Definition 3.1. A path is represented as $(x(s)), u(s)), s \in\left[0, s_{G}\right]$ and a trajectory is a time-parametrized path $(x(s(t))), u(s(t))), t \in\left[t_{I}, t_{G}\right]$.

Based on Definition 3.1, a trajectory can be constructed from a path by specifying velocity $\dot{s}(t)=v(t)$ of which the path should be executed. For path planning of ground vehicles, the direction of motion is already determined by the path planner and what remains for the velocity planner is to compute the desired longitudinal speed profile $|v(t)|$. For systems that are differentially flat, a motion plan can also be defined as a curve in the flat outputs (Paden et al., 2016). In that case, smoothness constraints on the curve have to be enforced such that the nominal state and control input trajectories can be recovered (Luca et al., 1998; Paden et al., 2016; Werling et al., 2012). 


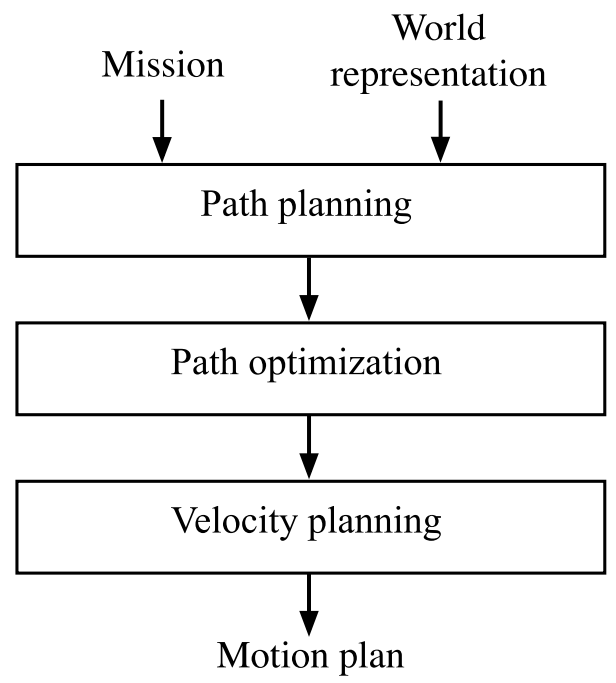

Figure 3.1: An example of typical steps that are performed to compute a motion plan.

\subsection{A common solution concept}

To solve, or rather to compute a suboptimal solution to the OCP in (3.2), motion planning algorithms are commonly used. A planning cycle for an autonomous vehicle is often divided into a couple of different phases, as illustrated in Figure 3.1 (LaValle, 2006; Lynch and Park, 2017). The perception and localization layer provides the motion planner with a compressed representation of the surrounding environment and information of the vehicle's current state. The high-level task planner provides the low-level motion planning and control layer with a desired mission to be executed. Based on these inputs, a motion plan is computed through a series of steps that are illustrated in Figure 3.1. First, a collision-free path from the vehicle's current state to a desired goal state is computed. In this planning phase, the system dynamics is sometimes neglected and the computed path is then not guaranteed to be feasible. The path can for example be composed of piecewise linear paths if a geometric planner is used during this step (LaValle, 1998, 2006; Likhachev et al., 2004). In the second step, the path is optimized, or smoothed, to generate a locally optimal motion plan that satisfies the system dynamics and its physically imposed constraints. Finally, a velocity profile is computed to obtain a trajectory. The velocity profile is computed such that the vehicle does not collide with any dynamic obstacle and such that the vehicle's maximum allowed velocities and accelerations, and other comfort and safety constraints are satisfied.

The nominal trajectory $(x(t), u(t)), t \in\left[t_{I}, t_{G}\right]$ is then sent to a feedback controller for plan execution. The planning cycle is then repeated in case a new mission is received or if the current motion plan has become infeasible due to, e.g., inaccurate prediction of dynamic obstacles or if a previously unseen obstacle is detected by the perception layer. 
Ideally, a unified motion planning algorithm that performs all three above mentioned steps simultaneously is preferred since separating the problem often leads to a suboptimal solution. This is however often intractable for most problem classes because of limited computation time. However, there exist efficient motion planning techniques where the vehicle's nonholonomic and physically imposed constraints are taken into account already while computing the path and some popular techniques will be presented in this chapter.

\subsection{Nonholonomic motion planning}

Motion planning for nonholonomic systems is conceptually not different compared to motion planning for a holonomic system, e.g., a quadcopter platform during low-speeds (Andersson et al., 2018b). The motion planning techniques that are presented here are therefore also applicable for holonomic systems, as long as the dimension of the state-space is sufficiently small and the algorithmic specific assumptions for the system dynamics are satisfied. However, the velocity constraints that are inherited by a nonholonomic system make different phases of the algorithms more demanding compared to the holonomic case.

For unstructured environments, e.g., parking lots and loading sites, a vast amount of different motion planning algorithms for ground vehicles exist and an overview can for example be found in LaValle (2006); Paden et al. (2016). Two popular branches for nonholonomic motion planning include probabilistic methods (Karaman and Frazzoli, 2011; Kuwata et al., 2009; LaValle and Kuffner, 2001) and deterministic methods (Cirillo et al., 2014; Pivtoraiko et al., 2009; Urmson et al., 2008) with different guarantees on the quality of the produced solution. Before presenting the planning algorithms, some important building blocks for these algorithms are presented.

\subsubsection{Steering functions}

Many motion planning algorithms require a method to generate a feasible trajectory between two nearby vehicle states (Karaman and Frazzoli, 2011; Pivtoraiko et al., 2009). Here, the surrounding obstacles are neglected and only the system dynamics $\dot{x}=f(x, u)$ and its physically imposed constraints $x \in \mathbb{X}$ and $u \in \mathbb{U}$ are considered. For some algorithms, this local trajectory generation problem needs to be solved online (Karaman and Frazzoli, 2011) and for others it is a procedure that is performed offline (Pivtoraiko et al., 2009). In the latter case, position invariance of the system is often exploited such that a precomputed trajectory can be translated and reused from all possible vehicle positions.

For unconstrained linear systems, Pontryagin's minimum principle (PMP) (Pontryagin, 2018) can be used to derive analytical solutions (Werling et al., 2012). PMP can also be used to derive analytical solutions for some nonlinear systems, including the kinematic bicycle model (2.11). Two examples are the Dubins (Dubins, 1957) and the Reeds-Shepp (Reeds and Shepp, 1990) minimal path lengths which have been extended to continuous and continuously differentiable vehicle curvature in (Fraichard and Scheuer, 2004) and (Oliveira et al., 2018), respectively. 
For systems that are differentially flat, efficient techniques for local trajectory generation can be used (Laumond et al., 1994; Murray and Sastry, 1991; Švestka and Vleugels, 1995; Tilbury et al., 1995). However, in all above examples, a general objective functional $J_{\mathrm{BVP}}$ is hard to minimize and feasibility with respect to physically imposed constraints on the vehicle need to be checked a posteriori (Frazzoli et al., 2005). In general, the problem of connecting two nearby vehicle states $x_{i} \in \mathbb{X}$ and $x_{f} \in \mathbb{X}$ without considering obstacles can be posed as an OCP in the following form

$$
\begin{array}{cl}
\underset{u(\cdot), t_{f}}{\operatorname{minimize}} & J_{\mathrm{BVP}}=\int_{0}^{t_{f}} L_{\mathrm{BVP}}(x(t), u(t)) \mathrm{d} t \\
\text { subject to } & \dot{x}(t)=f(x(t), u(t)), \\
& x(0)=x_{i}, \quad x\left(t_{f}\right)=x_{f}, \\
& x(t) \in \mathbb{X}, \quad u(t) \in \mathbb{U} .
\end{array}
$$

This OCP is sometimes referred to as a boundary value problem (BVP) and note its similarities to the formulation of the motion planning problem in (3.2). Here, the set of allowed vehicle states $\mathbb{X}$ is typically represented as a convex set, in contrast to (3.2) where $\mathbb{X}_{\text {free }}(t)$ is often non-convex. Moreover, as (3.3) is a subproblem of $(3.2)$, the optimal final time $t_{f}$ is also typically shorter. Together, these properties imply that (3.3) is easier to compute a locally optimal solution to. However, the OCP in (3.3) is still a non-convex problem due to the nonlinear system dynamics.

In many applications, it is important that $u(t)$ is sufficiently smooth which can be handled using an augmented state vector $\bar{x}(t)=\left(x(t), u(t), \dot{u}(t), \ldots, u^{(r-1)}(t)\right)$ and view the $r$-th time derivative $u^{(r)}(t)$ as an artificial control signal. It is now possible to penalize the derivatives of the nominal control signal and enforce limitations on them in a structured way. To guarantee that $u(t)$ is an $r-1$ times continuously differentiable function in time when multiple precomputed trajectories are combined during online planning, the boundary values for $\dot{u}(t), \ddot{u}(t), \ldots$, $u^{(r-1)}(t)$ are typically constrained to zero (Andersson et al., 2018b; Ljungqvist et al., 2019; Werling et al., 2012).

The solution to $(3.3)$ is a trajectory $(x(t), u(t)), t \in\left[0, t_{f}\right]$ that will be referred to as a motion primitive $m_{p}$ and the solution satisfies

$$
x\left(t_{f}\right)=x(0)+\int_{0}^{t_{f}} f(x(t), u(t)) \mathrm{d} t .
$$

Let $x_{k}=x(0)$ and $x_{k+1}=x\left(t_{f}\right)$, then the state transition equation in (3.4) is compactly defined as

$$
x_{k+1}=f_{p}\left(x_{k}, m_{p}\right),
$$

where the final vehicle state $x_{k+1}$ when applying $m_{p}$ from $x_{k}$ is called a successor state of $x_{k}$. The cost function $L_{\mathrm{BVP}}$ in (3.3) may be the same as the cost function $L$ 


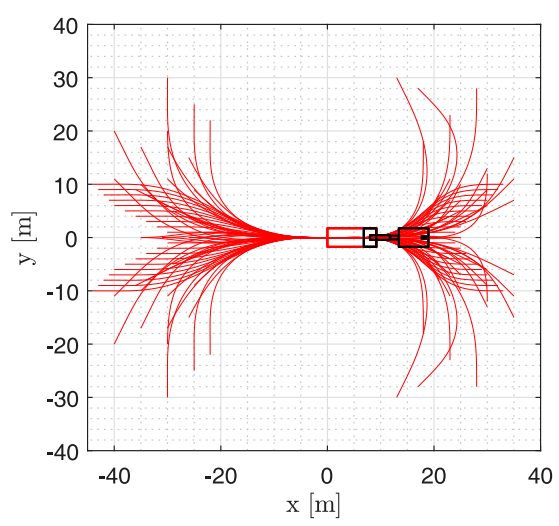

(a) Motions for a general 2-trailer with a car-like tractor from a straight configuration to different final states.

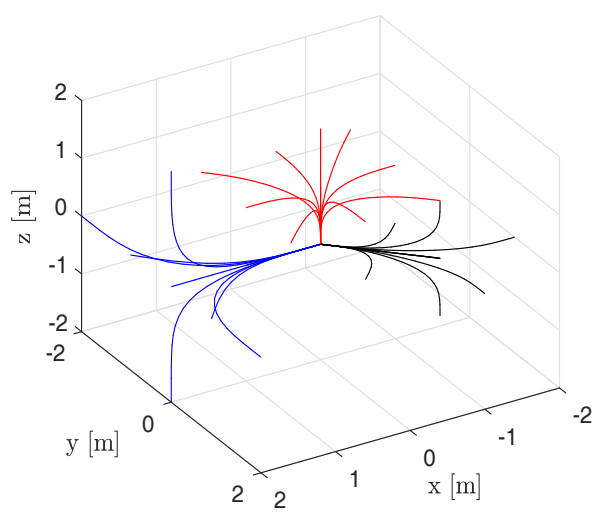

(b) Motions for a quadcopter platform from different initial velocities $v_{i}=$ $\left(v_{x, i}, v_{y, i}, v_{z, i}\right)$ to different final states.

Figure 3.2: Precomputed motions for a general 2-trailer with a car-like tractor (a) and for a quadcopter platform (b). Only the trajectories for the position of the rear axle of the semitrailer and the center of gravity for the quadcopter are plotted, respectively.

in the original motion planning problem in (3.2), or some of the terms are neglected in $L_{\mathrm{BVP}}$, e.g., only smoothness and comfort criteria are considered (Ljungqvist et al., 2017; Werling et al., 2012). In both cases, the stage cost $J\left(m_{p}\right)$ is computed by integrating the resulting trajectory using the cost function defined in the motion planning problem (3.2). Note however that from an optimality point of view it is preferred to use the same cost function in both steps (Bergman et al., 2019a).

One approach to find a locally optimal solution to the OCP in (3.3) is to use state-of-the-art numerical optimal control software, e.g., ACADO (Houska et al., 2011b) or CasADi (Andersson et al., 2018a). In these methods, the continuoustime optimal control problem is reformulated as a nonlinear programming (NLP) problem using for example direct multiple shooting combined with numerical integration. To solve the resulting NLP, a sequential quadratic programming (SQP) solver (Gill et al., 2005) or a nonlinear interior-point (IP) solver (Wächter and Biegler, 2006) can be used. Examples of different solutions for a general 2-trailer with a car-like tractor (see Section 2.4) and for a quadcopter platform (Andersson et al., 2018b) are provided in Figure 3.2. For systems with complex dynamics, it can be problematic to even find a feasible solution to the OCP in (3.3) and a proper initial guess is crucial. Moreover, systems with unstable dynamics can cause numerical problems while initializing the OCP solver. One such example is the general 2-trailer with a car-like tractor which is unstable in backward motion. In Paper D, we present a method to avoid this issue for tractor-trailer vehicles by establishing and exploiting time-symmetry properties of the system (Lamb and Roberts, 1998). 


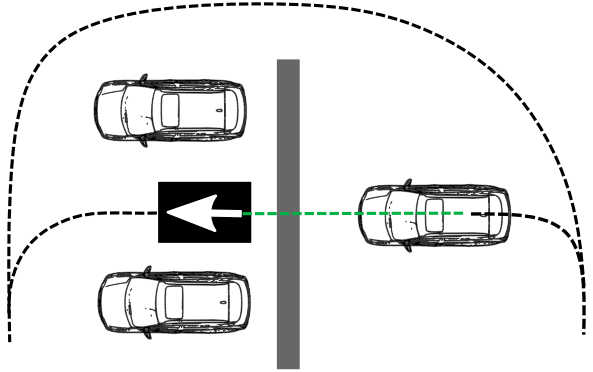

(a) The surrounding obstacles are not taken into account.

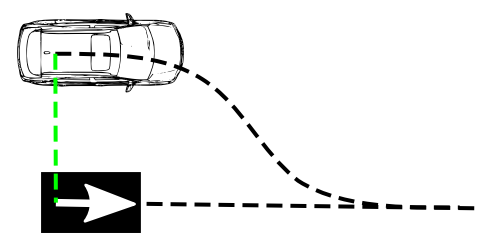

(b) The nonholonomic constraints are not taken into account.

Figure 3.3: Illustration of two cases when the Euclidean distance (green dashed line) severely underestimates the true cost-to-go (black dashed line).

\subsubsection{Heuristics}

All sampling-based motion planning algorithms incrementally explores a sampled set $\mathbb{X}_{s}$ of the vehicle's state-space $\mathbb{X}$. This exploration is either performed in a deterministic or in a probabilistic fashion with the common goal of computing a motion plan to the desired goal. During this search process, an informative heuristic function $h$ is needed to guide the exploration toward promising states (LaValle and Kuffner, 2001; Pivtoraiko et al., 2009). The heuristic function $h\left(x_{k}, x_{G}\right)$ is used to estimate the cost-to-go from a vehicle state $x_{k} \in \mathbb{X}_{s}$ to the goal state $x_{G} \in \mathbb{X}_{s}$. Let $h^{*}\left(x_{k}, x_{G}\right)$ denote the optimal cost-to-go for traveling from $x_{k}$ to $x_{G}$ and let $x_{k+1} \in \mathbb{X}_{s}$ represent a successor state of $x_{k}$ achieved using motion primitive $m_{p}$ with stage cost $J\left(m_{p}\right)$. A heuristic function $h\left(x_{k}, x_{G}\right)$ is said to be

Admissible if for all $x_{k} \in \mathbb{X}_{s}$, the heuristic function satisfies $h\left(x_{k}, x_{G}\right) \leq h^{*}\left(x_{k}, x_{G}\right)$

Consistent if for all $x_{k} \in \mathbb{X}_{s}$, and all its successor states $x_{k+1}$, the heuristic function satisfies $h\left(x_{k}, x_{G}\right) \leq J\left(m_{p}\right)+h\left(x_{k+1}, x_{G}\right)$

The admissibility criterion implies that the heuristic function always underestimates the optimal cost-to-go from $x_{k}$ to $x_{G}$ and the consistency criterion means that the heuristic cost from $x_{k}$ to $x_{G}$ cannot be larger than the stage cost $J\left(m_{p}\right)$ plus the heuristic cost from $x_{k+1}$ to $x_{G}$, i.e., the triangle inequality must hold. These criteria are necessary to satisfy in order to preserve optimality guarantees for many motion planning algorithms (LaValle, 2006).

When path planning is considered, the simplest heuristic function is the Euclidean distance. However, due to the nonholonomic constraints that are inherited by a nonholonomic system and the surrounding obstacles, the Euclidean distance may in many scenarios severely underestimate the true cost-to-go. Two illustrative examples for a car-like vehicle are presented in Figure 3.3. In Figure 3.3a, the surrounding obstacles are not taken into account for and in Figure 3.3b, the nonholonomic constraints make the Euclidean distance a poor estimator of the actual cost-to-go. Using a heuristic function that generates poor cost-to-go estimates 
causes the motion planner to spend unnecessary computation time on exploring vehicle states that seem promising according to the heuristics, but are in reality bad candidates since the true cost-to-go from these states may be very expensive.

A common approach when designing heuristic functions is to relax the motion planning problem by removing certain constraints to make the problem simpler to solve. For car-like vehicles, the problem in Figure 3.3b can be handled by neglecting obstacles and compute the analytical solution for the Reeds-Shepp car (Reeds and Shepp, 1990) and use its optimal path-length as heuristic function. For the case in Figure 3.3a, the system dynamics can be removed and a geometric planning problem could be solved to compute an underestimate of the cost-to-go. These two strategies are combined in Dolgov et al. (2010) where first a local trajectory generation problem without obstacles is solved, then the system dynamics is neglected and a geometric planning problem with obstacles is solved and the maximum of these two estimates is used as heuristic cost. At a first glance, this proposal may seem computationally expensive for nonlinear systems since a local trajectory generation problem without obstacles involves solving an OCP (3.3) at each state expansion. Fortunately, this problem can in many applications be performed offline on a finite grid of vehicle states and stored in a heuristic look-up table (HLUT) (Cirillo et al., 2014; Knepper and Kelly, 2006). The HLUT can then be used during online planning to efficiently retrieve the heuristic function values for many problems in the vicinity of the vehicle.

Depending on the complexity of the surrounding environment, the simplified geometric planning problem may yield a good or a poor estimate of the cost-to-go. For the problem in Figure 3.3b, the Euclidean distance and the solution to the geometric planning problem will yield the same poor estimate of the cost-to-go. For maze-like environments as in Figure 3.3a, the solution to a geometric planning problem will result in a good estimate of the cost-to-go and will guide the motion planning algorithm to not waste computation time on expanding states that lead into dead ends. To conclude, the development of a good and computationally efficient heuristic function is application dependent and depends on the surrounding environment the vehicle is intended to operate in.

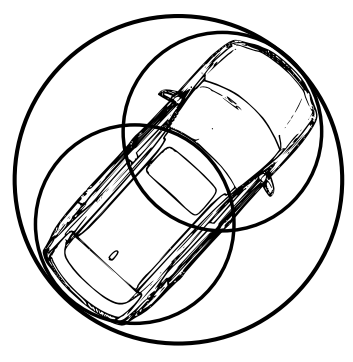

(a)

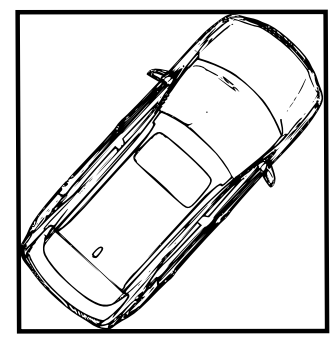

(b)

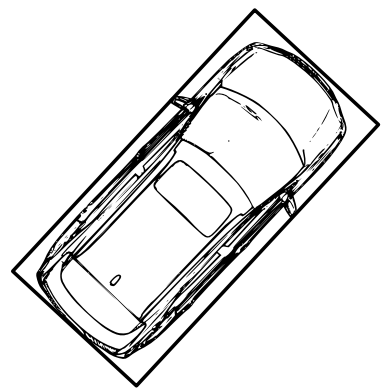

(c)

Figure 3.4: Three different kinds of bounding regions: (a) circles, (b) axisaligned bounding box, and (c) oriented bounding box. 


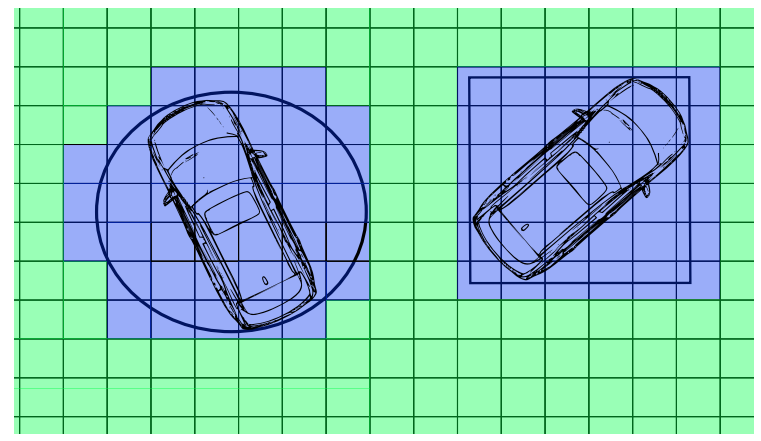

Figure 3.5: Illustration of how obstacles with different bounding boxes are transformed to a binary occupancy grid map where blue means occupied and green means unoccupied.

\subsubsection{Collision detection}

All motion planning algorithms rely on efficient collision detection methods. The choice of method depends on how the surrounding obstacles $\mathbb{X}_{\text {obs }}(t)$ are represented and is in general invariant to the choice of motion planning algorithm. Typically, static obstacles $\mathbb{X}_{\text {obs }}^{s}$ and dynamic obstacles $\mathbb{X}_{\text {obs }}^{d}(t)$ are treated separately. Since the trajectory for a dynamic obstacle is unknown (unless the vehicles are communicating), its motion has to be predicted. The predictions are usually performed by a tracking and prediction module in the perception layer where probabilistic state-space models are used for trajectory prediction of the dynamic obstacles. The details of these algorithms are out of the scope of this thesis and the reader is referred to, e.g., Deo and Trivedi (2018); Deo et al. (2018); Houenou et al. (2013); Kim et al. (2017) and references therein for details.

Collision detection for static obstacles are typically performed using hierarchical methods where different bounding regions are used in each step. Figure 3.4 illustrates four different kinds of bounding regions: a bounding circle, multiple bounding circles, an axis-aligned bounding box (AABB) and an oriented bounding box (OBB). The complexity of performing collision checking for each type of bounding region is in increasing order where a circle is cheap and an oriented bounding box is costly. If the controlled vehicle is also represented as a bounding region (or a hierarchy of bounding regions), the criterion for collision is that the regions are intersecting. If the bounding circles are overlapping, AABB may be checked and finally OBB. As soon as one step reports no collision, the vehicle state is declared collision free. For multi-body vehicle's, separate bounding regions for each body are typically used (LaValle, 2006).

The collision detection procedure has to be performed thousands of times in a single planning cycle and thus precomputing certain steps significantly speeds up the planning process. A common way to perform $2 \mathrm{D}$ collision detection is to compute an occupancy grid map (Elfes, 1989) or an OctoMap for the 3D case (Hornung et al., 2013). The grid map is divided into cells where the obstacles' bounding 


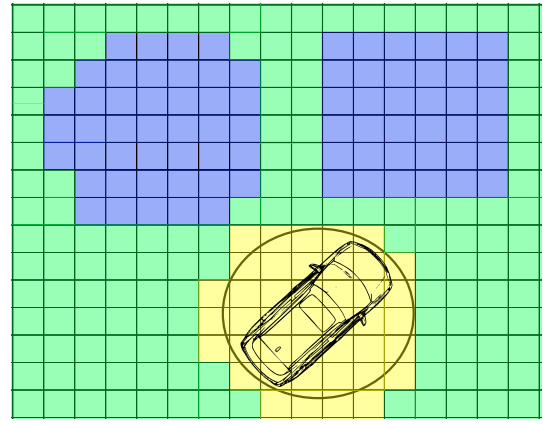

(a)

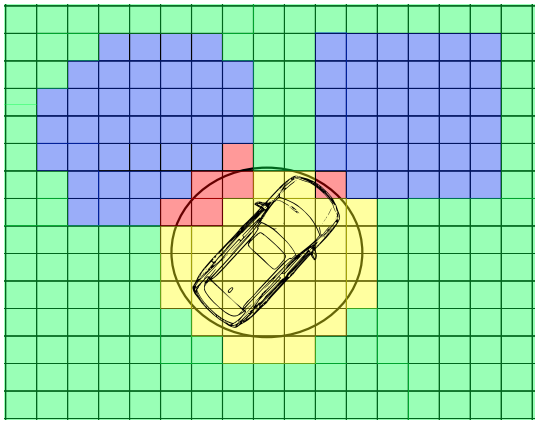

(b)

Figure 3.6: Collision detection using a binary occupancy grid map where a red cell means that the bounding circle of the controlled vehicle is intersecting an obstacle. In (a) the controlled vehicle is not in collision with any obstacle and in (b) the controlled vehicle is in collision with both obstacles.

regions are used to classify a cell as free or occupied ${ }^{1}$. Figure 3.5 illustrates how an occupancy gridmap is computed where a green cell means that the cell is free and a blue cell means that the cell is occupied by an obstacle. The occupancy grid map can then be used to efficiently perform collision detection with the bounding region of the controlled vehicle, as illustrated in Figure 3.6. In Figure 3.6a, the vehicle is in free space $x \in \mathbb{X}_{\text {free }}$, whereas in Figure 3.6b the vehicle is in collision with an obstacle $x \in \mathbb{X}_{\mathrm{obs}}^{s}$ and therefore not in free space $x \notin \mathbb{X}_{\text {free }}$. Note that the collision detection procedure may be rather conservative if a single circle is used as bounding region.

Collision detection of a motion primitive $m_{p}$ is treated in analogy to a single state except that all states along the trajectory have to be collision free. In practice, only a finite number of states along the trajectory are used for collision detection and a motion primitive $m_{p}$ applied from initial state $x_{k}$ is declared collision free if $c\left(x_{k}, m_{p}\right) \in \mathbb{X}_{\text {free }}$, otherwise it is declared as in collision. If the motion primitives are precomputed, it is possible to speed up collision detection by precomputing and storing the vehicle's swept area as a grid map (called swath) for each maneuver (Pivtoraiko et al., 2009).

\subsection{Sampling-based motion planning}

We are now ready to present a general sampling-based motion planning framework for nonholonomic systems. For simplicity, only static obstacles are considered in the remainder of this chapter, i.e., $\mathbb{X}_{\text {obs }}^{d}(t)=\emptyset, \forall t$. A sampling-based motion planner performs its search process in a directed ${ }^{2}$ search graph $\mathcal{G}=\langle\mathcal{V}, \mathcal{E}\rangle$ where a vertex $x_{k} \in \mathcal{V}$ represents a vehicle state and an edge $e_{k} \in \mathcal{E}$ encodes a motion

\footnotetext{
${ }^{1}$ Probabilistic information and distances to obstacles can be incorporated in the cells instead of a binary representation (Urmson et al., 2008).

${ }^{2}$ It is also possible to use a bidirectional or an undirected search graph where the planner is searching from $x_{I}$ to $x_{G}$, from $x_{G}$ to $x_{I}$ and possibly from other states as well at the same time.
} 
primitive $m_{p}$, i.e., a feasible trajectory or path, that connects two vehicle states. The search graph can be incrementally constructed online or parts of the graph are precomputed offline. The general search process to compute a motion plan from the vehicle's initial state $x_{I} \in \mathbb{X}_{\text {free }}$ to its desired goal state $x_{G} \in \mathbb{X}_{\text {free }}$ involves the following main steps (LaValle, 2006):

1. Initialization: Let $\mathcal{G}=\langle\mathcal{V}, \mathcal{E}\rangle$ represent a directed search graph, for which only $x_{I} \in \mathbb{X}_{\text {free }}$ is contained in the vertex set $\mathcal{V}$ and the edge set $\mathcal{E}$ is empty. The cost-to-come is $J\left(x_{I}, x_{I}\right)=0$ and the estimated cost-to-go is $h\left(x_{I}, x_{G}\right)$.

2. Select expansion candidate: Select a promising vehicle state $x_{k} \in \mathcal{V}$ by sorting $\mathcal{V}$ based on, e.g., estimated total cost $J\left(x_{I}, x_{k}\right)+h\left(x_{k}, x_{G}\right)$, and a desired vehicle state $x_{k+1} \in \mathbb{X}_{\text {free }}$ to connect.

3. Steering function: Try to generate a motion primitive $m_{p}$ from $x_{k}$ to $x_{k+1}$ by solving an OCP as in (3.3) such that the state transition $x_{k+1}=$ $f_{p}\left(x_{k}, m_{p}\right)$ is achieved and the resulting trajectory is collision free $c\left(x_{k}, m_{p}\right) \in$ $\mathbb{X}_{\text {free. }}$ If this stage fails, then go back to Step 2.

4. Insert in graph: If $x_{k+1} \notin \mathcal{V}$, it is added with cost-to-come $J\left(x_{I}, x_{k+1}\right)=$ $J\left(x_{I}, x_{k}\right)+J\left(m_{p}\right)$. If $x_{k+1}$ already exists in $\mathcal{V}$ and $J\left(x_{I}, x_{k+1}\right)$ is more expensive, $x_{k+1}$ is not inserted. On the contrary, if $J\left(x_{I}, x_{k+1}\right)$ is cheaper, a rewiring step may be performed where the current edge to $x_{k+1}$ is replaced with the new edge.

5. Check for a solution: If $x_{k+1}=x_{G}$ ( or $x_{k+1} \in \mathbb{X}_{G}$ ), backtrace from $x_{k+1}$ to $x_{I}$ and mark its trajectory as a solution candidate with cost $J\left(x_{I}, x_{k+1}\right)$.

6. Check for termination: Go back to Step 2 unless a termination condition is satisfied. Otherwise, if multiple solution candidates are found, return the solution with minimum total cost $J$ or return failure if no solution has been found.

With minor modifications, most sampling-based motion planning algorithms follow the above mentioned steps. Two motion planning algorithms will now be presented, but first, some properties for motion planning algorithms are defined. A motion planning algorithm is said to be (LaValle, 2006):

Complete if the algorithm always finds a solution in finite time if one exists or terminates and reports failure if one does not exist.

Resolution complete if the algorithm always finds a solution within a specified resolution in finite time if one exists or terminates and reports failure if one does not exist.

Probabilistically complete if a solution exists, the probability of finding a solution approaches one as the number of iterations tends to infinity.

Completeness is an attractive property for any motion planning algorithm. However, when motion planning for nonholonomic systems is considered, the completeness property is hard to establish for an algorithm and weaker properties, such as resolution or probabilistic completeness, are often considered sufficient. 


\subsubsection{Motion planning in state lattices}

The main idea with lattice-based motion planning is to precompute the building blocks for a directed search graph $\mathcal{G}=\langle\mathcal{V}, \mathcal{E}\rangle$ offline. These building blocks consist of vertices and edges that, when repeated, generate a search graph $\mathcal{G}$ that forms a regular and repeated pattern. This search graph often called a state lattice or a lattice graph, and thanks to its structure enables efficient graph search algorithms to be used during online planning (Cirillo et al., 2014; Pivtoraiko et al., 2009).

The state lattice is constructed offline by discretizing the vehicle's state space $x_{k} \in \mathcal{X}_{d}$ and computing a set of motion primitives $\mathcal{P}$ by solving a large number of OCPs in the form of (3.3). Each vertex $v_{k} \in \mathcal{V}$ is a vehicle state $x_{k} \in \mathcal{X}_{d}$ and each edge $e_{k} \in \mathcal{E}$ represents a motion primitive $m_{p} \in \mathcal{P}$. Each motion primitive $m_{p} \in \mathcal{P}$ is a feasible trajectory (3.4) that moves the vehicle from one discrete vehicle state $x_{k} \in \mathcal{X}_{d}$ to another $x_{k+1} \in \mathcal{X}_{d}$ in a bounded neighborhood in freespace. In other words, the initial and final states of a motion primitive must comply with the specified state-space discretization. The framework assumes that the vehicle is position invariant which implies that the set of motion primitives $\mathcal{P}$ can be computed from the position of the vehicle at the origin. A motion primitive can then be translated and reused from all other discrete positions during online planning. In general, all motion primitives are not applicable from each vehicle state $x_{k} \in \mathcal{X}_{d}$ and the motion primitives that can be used from $x_{k}$ is denoted $\mathcal{P}\left(x_{k}\right) \subseteq \mathcal{P}$. The average size of $\mathcal{P}\left(x_{k}\right)$ represents the branching factor of the search problem and with increasing number of edges from each $x_{k} \in \mathcal{X}_{d}$, a good heuristic function becomes increasingly important during online planning.

By specifying the discretized state space $\mathcal{X}_{d}$ and precomputing the set of motion primitives $\mathcal{P}$, the continuous-time motion planning problem in (3.2) is approximated by a discrete-time OCP in the form

$$
\begin{array}{cl}
\underset{\left\{m_{p}^{k}\right\}_{k=0}^{N-1}, N}{\operatorname{minimize}} & J_{\mathrm{DP}}=\sum_{k=0}^{N-1} J\left(m_{p}^{k}\right) \\
\text { subject to } & x_{0}=x_{I}, \quad x_{N}=x_{G}, \\
& x_{k+1}=f_{p}\left(x_{k}, m_{p}^{k}\right) \\
& m_{p}^{k} \in \mathcal{P}\left(x_{k}\right) \\
& c\left(m_{p}^{k}, x_{k}\right) \in \mathbb{X}_{\text {free }} .
\end{array}
$$

The decision variables to the OCP in (3.6) are the motion primitive sequence $\left\{m_{p}^{k}\right\}_{k=0}^{N-1}$ and its length $N$. The set of applicable motion primitives from a certain vehicle state $x_{k}$ is encoded in $(3.6 \mathrm{~d})$ and a state transition $x_{k+1}=f_{p}\left(x_{k}, m_{p}^{k}\right)$ is feasible if the resulting trajectory remains in free space (3.6e). In (3.6), it is assumed that $x_{I} \in \mathcal{X}_{d}$ and $x_{G} \in \mathcal{X}_{d}$ to make the problem well defined. If $x_{I} \notin \mathcal{X}_{d}$ or $x_{G} \notin \mathcal{X}_{d}$, they have to be projected to their closest neighboring state in $\mathcal{X}_{d}$ using some distance metric. The projection of $x_{I}$ to $\mathcal{X}_{d}$ should take into account the region of attraction for the closed-loop system, consisting of the feedback controller and the controlled vehicle. Otherwise, successful execution of the motion plan cannot be guaranteed. One approach to alleviate this problem is to include a post- 


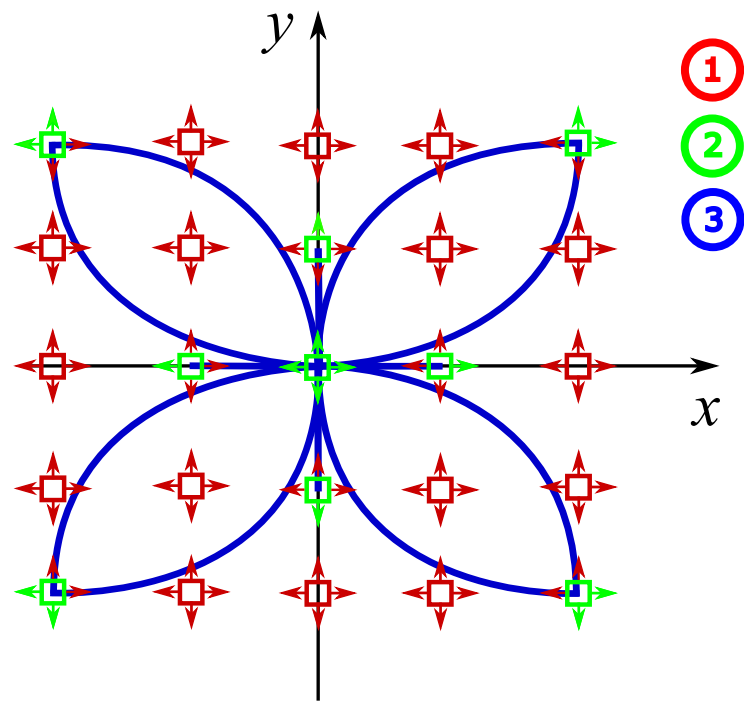

Figure 3.7: An illustration of the three steps that are performed to generate the state lattice. (1) Discretize the state-space, (2) select which states to connect, (3) solve the BPVs.

optimization step (Bergman et al., 2019a), where an OCP solver is warm-started with the solution computed by the lattice planner. In this framework, the lattice planner is used to solve the combinatorial aspects of the problem, e.g., to pass an obstacle to the left or to the right, and the OCP solver is then used to compute a locally optimal solution to the motion planning problem that starts at the vehicle's initial state and reaches the goal state exactly. In Paper F, this technique is used to develop a trajectory planner for general N-trailer vehicles that can have a mixture of passively and actively steered trailers.

The construction of the state lattice is now explained more thoroughly.

\section{State lattice construction}

The offline construction of the state lattice is illustrated in Figure 3.7 and can be divided into three separate steps:

1. Specify the resolution of the search space $\mathcal{V}$ by discretizing the vehicle's state-space.

2. Select which pairs of discrete vehicle states to connect based on the reachability of the system.

3. Generate the set of motion primitives $\mathcal{P}$ by solving the OCPs defined in the previous step.

In the first step, the vehicle's state-space is discretized based on the maneuverability of the system and the environment in which the vehicle is intended to operate. 


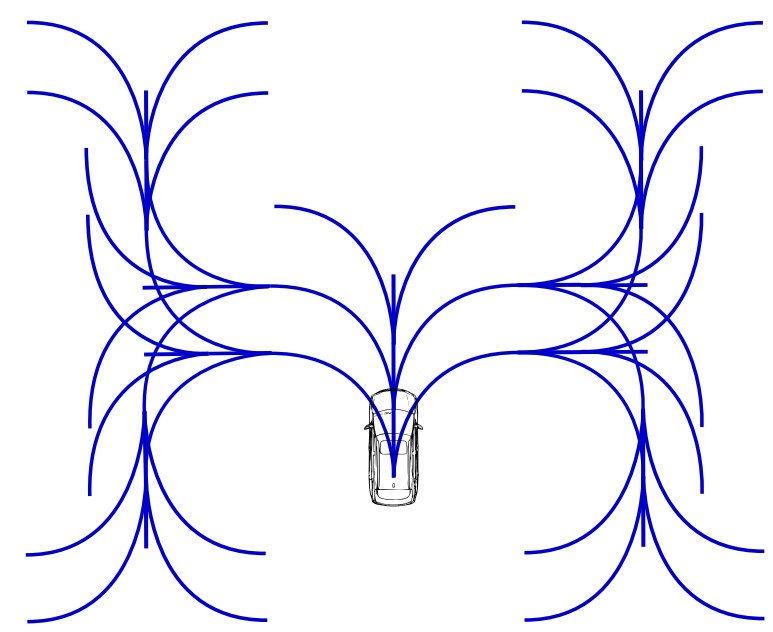

Figure 3.8: The first three levels of the reachability graph $\mathcal{G}_{r}\left(x_{k}, \mathcal{P}\right)$ from $x_{k}=\left[\begin{array}{lll}0 & 0 & \pi / 2\end{array}\right]^{\top}$ where the vehicle is restricted to move according to the motion primitives in Example 3.1.

For path planning of a car-like vehicle, the first step is to specify the fidelity of the vehicle's position, orientation and steering angle (Pivtoraiko et al., 2009).

The second step is to select which pairs of discrete vehicle states to connect. At this step, it is important to be aware of that the complexity of the discrete-time OCP in (3.6) scales exponentially in the number of applicable motion primitives $\mathcal{P}\left(x_{k}\right)$ from each discrete vehicle state $x_{k}$. Therefore, a careful selection of vehicle states to connect is most often performed. The selection should be performed such that the resulting reachability graph $\mathcal{G}_{r}\left(x_{k}, \mathcal{P}\right)$ (the set of all possible trajectories from $x_{k}$ given the motion primitive set $\mathcal{P}$ ) sufficiently resembles the vehicle's forward reachable set from $x_{k}$.

The third step is to solve the specified OCPs, e.g., using some of the optimization methods presented in Section 3.4.1 or to employ the maneuver-based motion primitive generation framework presented in Bergman et al. (2019b). Examples of motion primitives $\mathcal{P}\left(x_{k}\right)$ from a selection of vehicle states $x_{k}$ for a general 2-trailer with a car-like tractor and for a quadcopter platform are depicted in Figure 3.2 where CasADi (Andersson et al., 2018a) together with IPOPT (Wächter and Biegler, 2006) are used to solve the resulting OCPs. To clarify the above mentioned steps, Example 3.1 shows how to construct a minimal state lattice for a kinematic bicycle model.

\section{Example 3.1: The Kinematic bicycle model}

The vehicle states for the Kinematic bicycle model $(2.11)$ are the position $\left(x_{1}, y_{1}\right) \in$ $\mathbb{R}^{2}$ and orientation $\theta_{1} \in(-\pi, \pi]$. The control signals are the steering angle $\alpha$ and longitudinal velocity $v=1$, i.e., the vehicle is only allowed to move forward with constant longitudinal velocity. The position of the vehicle is discretized to a uniform grid with resolution $r$ in both $x$ and $y$. The orientation is discretized 
into four different orientations $\Theta=\{-\pi / 2,0, \pi / 2, \pi\}$. The steering angle $\alpha$ is constrained to zero at each vertex to make sure that it is continuous when multiple edges are combined during online planning.

The pair of discrete vehicle states to connect are illustrated in Figure 3.7 where a green box with a green arrow encodes that a discretized vehicle state is included. The resulting motion primitives are the blue paths where only $\pm 90^{\circ}$-turns and straight forward motions are generated from each initial orientation $\theta_{i} \in \Theta$ from the position of the vehicle at the origin. The first three levels of the resulting reachability graph $\mathcal{G}_{r}\left(x_{k}, \mathcal{P}\right)$ from $x_{k}=\left[\begin{array}{lll}0 & 0 & \pi / 2\end{array}\right]^{\top}$ are illustrated in Figure 3.8.

To improve the online planning time, the motion primitive set $\mathcal{P}$ can be reduced using the reduction technique presented in Cirillo et al. (2014); Pivtoraiko and Kelly (2011). A motion primitive $m_{p} \in \mathcal{P}$ with stage cost $J\left(m_{p}\right)$ is removed if its state transition can be achieved by a combination of the other motion primitives in $\mathcal{P}$ with a combined total stage cost $J_{\text {comb }}$ that satisfies $J_{\text {comb }} \leq \eta J\left(m_{p}\right)$, where $\eta \geq 1$ is a user specified constant. This procedure can also be used to verify that redundant motion primitives do not exist by selecting $\eta=1$. Moreover, as discussed in Section 3.4.2, an informative heuristic function $h$ is needed to guide the search algorithm during online planning where, e.g., a precomputed HLUT can be used to quickly access the heuristic cost at runtime (Cirillo et al., 2014; Pivtoraiko et al., 2009).

\section{Online planning}

During online planning, the discrete-time OCP in (3.6) is solved using a graphsearch algorithm, such as, A* (Hart et al., 1968) or anytime repairing A* ARA $^{*}$ ) (Likhachev et al., 2004). Since these methods perform deterministic searches in a directed search graph, the lattice planner automatically inherits the properties and guarantees of the chosen graph-search algorithm. The solution to the discretetime OCP in (3.6) is an ordered sequence of $N$ motion primitives $\left\{m_{p}^{k}\right\}_{k=0}^{N-1}$, i.e., a feasible and collision-free trajectory $(x(t), u(t)), t \in\left[t_{I}, t_{G}\right]$ or path $(x(s), u(s))$, $s \in\left[0, s_{G}\right]$ that minimizes the objective function $J_{\mathrm{DP}}$. If $\mathrm{A}^{*}$ is used as search method and enough planning time is given, the algorithm is guaranteed to return a solution that is resolution optimal (Pivtoraiko et al., 2009).

The $A^{*}$ graph-search algorithm is presented in Algorithm 1. With some minor adjustments the algorithm follows the steps presented in Section 3.5. Firstly, since the motion primitive set is precomputed, no OCP needs to be solved online. Secondly, not only one but all possible edges $\mathcal{P}\left(x_{k}\right)$ from the best candidate vertex $x_{k}$ are explored. In the algorithm, the closed Set stores the discrete vehicle states that have been expanded from and the openSet stores the vehicle states that have been explored but remains to be expanded. The openSet is commonly referred to as the frontier and is typically implemented as a priority queue. If the heuristic function $h$ is admissible, the termination condition is trivial (line 10). That is, if the best candidate vertex $x_{k}$ from the openSet is $x_{k}=x_{G}$, the optimal motion primitive sequence $\left\{m_{p}^{k}\right\}_{k=0}^{N^{*}-1}$ is found with cost $J\left(x_{k}, x_{I}\right)$ and the motion primitive sequence can then be obtained by backtracing from the found goal node $x_{k}$ 


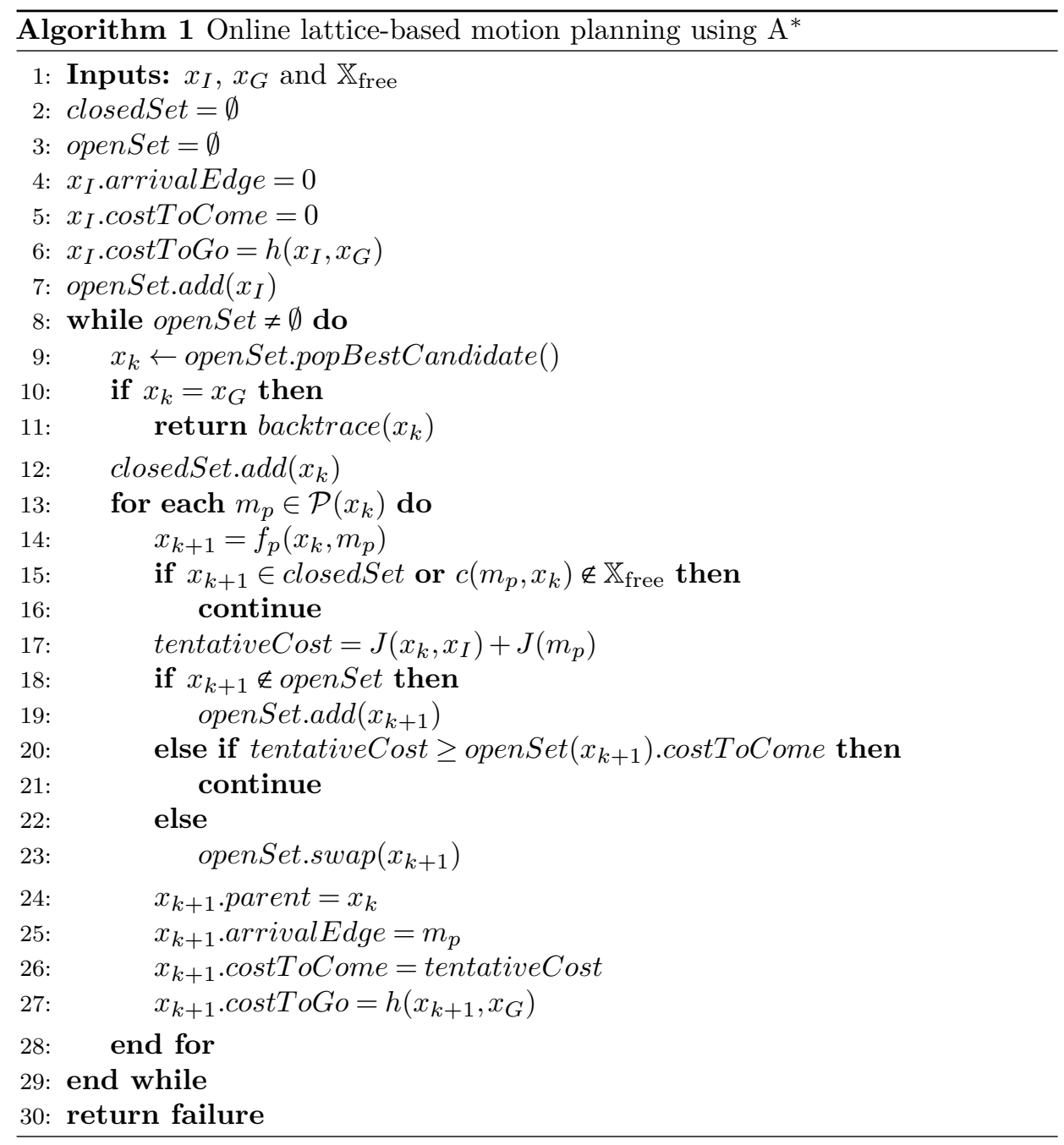

to the root node $x_{I}$ (line 11). Moreover, the principle of optimality ensures that every subtrajectory is optimal for its corresponding subproblem.

If $x_{G} \notin \mathcal{X}_{d}$ and an accurate goal state $x_{G}$ is important, an OCP in the form of (3.3) can to be solved online to optimize the final part of the motion plan $(x(t), u(t)), t \in\left[t_{I}, t_{G}\right]$ such that the goal state is reached exactly. The same approach can be used for the initial state $x_{I}$ but is often omitted and the initial transient is left for the feedback controller to suppress.

Due to the algorithm's efficiency, lattice-based motion planning has been successfully deployed on various robotic platforms (Andersson et al., 2018b; Pivtoraiko et al., 2009; Urmson et al., 2008). To illustrate the capability of lattice-based motion planners, two planning examples are illustrated in Figure 3.9. In Figure 3.9a, the quadcopter problem considered in Andersson et al. (2018b) is shown and in Figure $3.9 \mathrm{~b}$, the general 2-trailer vehicle from Paper D is shown. 


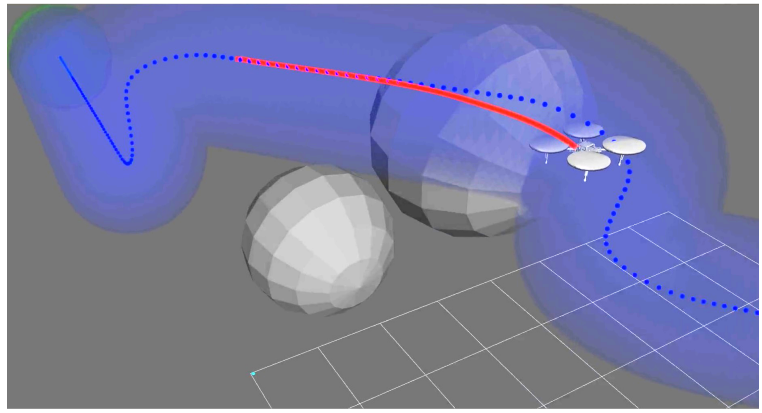

(a)

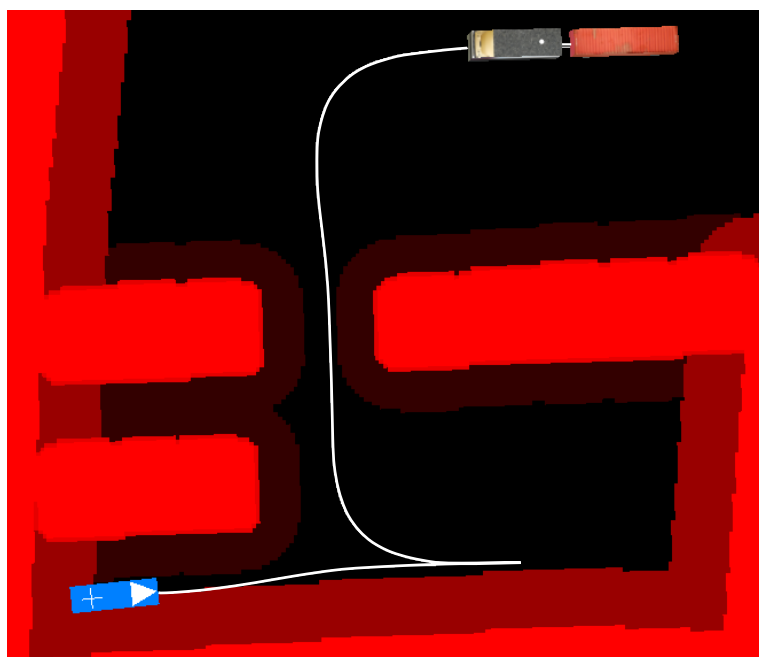

(b)

Figure 3.9: Two online planning examples using a lattice-based motion planner. In (a), trajectory planning for a quadcopter platform is shown and in (b), path planning for a general 2-trailer with a car-like tractor is shown. The blue and the white paths are the computed motion plans for the position of the quadcopter and the position for the axle of the semitrailer, respectively.

\section{Other deterministic approaches}

There exist other deterministic motion planning algorithms that rely on controlinput sampling $u \in \mathcal{U}_{d}$ instead of state-space discretization. In this case, a model of the vehicle is used online to simulate the system for $\Delta t$ seconds, or $\Delta s$ meters, using a constant or parametrized control signal $u(t), t \in[0, \Delta t]$ from $\mathcal{U}_{d}$. In general, the resulting trajectory segment does not end up at a specified final state. This implies that the search graph becomes irregular which results in a frontier that grows exponentially during online graph search (Paden et al., 2016; Pivtoraiko et al., 2009). To resolve this, the vehicle's state-space is often divided into cells where a cell is only allowed to contain one state. One motion planning algorithm that 
uses control-input sampling is the hybrid A* (Dolgov et al., 2010). Common for these approaches is that they lack all completeness and optimality guarantees and control-input sampling is not practical for unstable systems, unless the simulations are performed in closed-loop with a stabilizing feedback controller.

On the contrary, control-input sampling may be more suitable for systems that are not position invariant or if some vehicle states (except for the position of the vehicle) have to be discretized with high resolution. The latter case arise in dynamic on-road driving scenarios where trajectory planning is needed. A statelattice approach is here impractical to use since the discretization of a sufficient number of possible vehicle speeds will result in a huge search graph during online planning (Ziegler and Stiller, 2009). An efficient algorithm that is tailored for on-road driving is the so-called spatiotemporal lattice formulation (McNaughton et al., 2011; Ziegler and Stiller, 2009) where parts of the vehicle's state-space is regularly discretized offline and other parts are implicitly discretized during online planning by sampling the vehicle's control inputs.

\subsubsection{Motion planning using RRT}

Another popular family of motion planning algorithms includes the probabilistic rapidly-exploring random tree (RRT) algorithm (LaValle, 1998). Only a brief overview will be presented and the reader is referred to, e.g., Karaman and Frazzoli (2010); LaValle (2006); Paden et al. (2016) for more details. In contrast to the deterministic approaches presented in the previous section, the RRT algorithm is based on randomized exploration of the search space. The original RRT algorithm was developed for holonomic systems and was later extended to nonholonomic systems in LaValle and Kuffner (2001) where the algorithms follow the main steps presented in Section 3.5.

The RRT algorithm incrementally constructs a directed search tree ${ }^{3}$ during online planning. The search tree is expanded by iteratively drawing random samples $x_{\text {rand }} \in \mathbb{X}$. A steering function is then used to connect from its nearest neighboring state $x_{\text {near }}$ in the tree towards $x_{\text {rand }}$. If the resulting edge is collisionfree, a new vertex is added to the tree where $x_{\text {near }}$ is determined by sorting the vertices in the search tree using a heuristic function, e.g., a look-up table for a nonholonomic system (LaValle and Kuffner, 2001; Pivtoraiko et al., 2009). If an exact steering function exists, the RRT algorithm can connect to a specified goal state $x_{G}$ by performing goal sampling. Otherwise, the desired goal state has to be replaced with a goal region $\mathbb{X}_{G}$, e.g., a ball centered around the goal state: $X_{G}=\left\{x \in \mathbb{X} \mid\left\|x-x_{G}\right\|_{Q} \leq \epsilon\right\}$ where $Q \succ 0$ and $\epsilon>0$.

For holonomic systems, the RRT algorithm is probabilistically complete, but is also guaranteed with probability one to converge to a suboptimal solution (Karaman and Frazzoli, 2010). For the nonholonomic case, the RRT algorithm lacks all completeness guarantees (Karaman and Frazzoli, 2010; Kunz and Stilman, 2015). An extension of the basic RRT is the so-called RRT* (Karaman and Frazzoli, 2011) where a rewiring procedure is performed in each tree expansion. In this step, the edges for the neighboring vertices in a ball around the new sample is

\footnotetext{
${ }^{3} \mathrm{~A}$ search tree is a special type of a search graph where each vertex only has one parent.
} 
rewired if a lower cost-to-come can be achieved. With this extension, $\mathrm{RRT}^{*}$ is shown to be probabilistically optimal meaning that the algorithm will converge to an optimal solution when the number of samples approaches infinity. However, the rewiring procedure involves solving several OCPs online. Therefore, its practical applicability is limited to systems where analytical steering functions exist.

Many modifications to the original RRT algorithm have been proposed to speed up the search using different pruning strategies and heuristic functions that bias the sampling towards interesting regions. These methods are out of the scope of this thesis and some extensions can be found in, e.g., Gammell (2017); Kalisiak and van de Panne (2006); Nasir et al. (2013); Urmson and Simmons (2003).

Anytime implementations of RRTs have been successfully deployed in dynamical environments by Kuwata et al. (2009) using biased sampling of reference signals to a closed-loop system. The closed-loop RRT (CL-RRT) algorithm was developed to enable a longer tree expansion without solving expensive OCPs online, i.e., the stabilizing controller is acting as an inexact solver. Moreover, the CL-RRT framework is suitable for unstable systems which is exploited in Paper A where a motion planner for a reversing general 2-trailer with a car-like tractor is presented. 


\section{Feedback control for self-driving vehicles}

This chapter presents different feedback-control techniques for self-driving vehicles. In this context, the objective of the feedback controller is to ensure that the controlled vehicle executes a computed motion plan with a small control error even if disturbances and initial control errors may be present. Depending on the reference obtained from the motion planner, the feedback-control problem will either be referred to as a path-following control problem or a trajectory-tracking control problem. We recall that the vehicle is assumed to be modeled as a time-invariant nonlinear system $\dot{x}(t)=f(x(t), u(t))$. During the control design, it is assumed that the vehicle state $x(t)$ is known ${ }^{1}$ and that the system is controllable, at least locally around the nominal trajectory or path (Khalil and Grizzle, 2002). Moreover, the motion plan is assumed to be feasible and collision-free, i.e., it satisfies the vehicle model and does not collide with any surrounding obstacles. Here, the theory will be presented in continuous-time but most of the material has a discrete-time counterpart (Anderson and Moore, 2007; Garcia et al., 1989; Rawlings, 2000).

The remainder of this chapter is structured as follows. In Section 4.1, the trajectory-tracking and path-following control concepts are explained. In Section 4.2, the trajectory-tracking problem is formally derived and some controller synthesis techniques are presented. In Section 4.3, path following for wheeled vehicles is presented together with some path-following techniques tailored for selfdriving vehicles.

\subsection{Problem formulations}

The objective of the feedback controller is to stabilize the vehicle around a nominal path or trajectory computed by a motion planner. Even though the control tasks

\footnotetext{
${ }^{1}$ In practice, the vehicle states are estimated using information from sensors on the vehicle. The sensor information is fused using nonlinear observers to obtain a state estimate $\hat{x}(t) \approx x(t)$.
} 
differ, the solution is in the form of a state-feedback controller. The control tasks are defined as follows:

Trajectory tracking: The closed-loop system consisting of the controlled vehicle and the trajectory-tracking controller should execute a nominal trajectory $\left(x_{r}(t), u_{r}(t)\right), t \in[0, \infty]$ with a small tracking error $\bar{x}(t)=x(t)-x_{r}(t)$.

Path following: The closed-loop system consisting of the controlled vehicle and the path-following controller should execute a nominal path $\left(x_{r}(s), u_{r}(s)\right)$, $s \in[0, \infty]$ with a small path-following error $\tilde{x}(t)=x(t)-x_{r}(s(t))$. Here, the path parameter $s(t)$, satisfying $\dot{s}>0$, is defined as the progression of the vehicle along the nominal path.

In trajectory tracking, the reference trajectory consists of nominal vehicle states $x_{r}(t)$ and control inputs $u_{r}(t)$ that are parametrized in time. In other words, the objective of the trajectory-tracking controller is to stabilize the vehicle around a nominal state trajectory. Here, the nominal trajectory $\left(x_{r}(\cdot), u_{r}(\cdot)\right)$ at each time instant is predetermined and is independent of the vehicle's current state $x(t)$. The trajectory-tracking problem is illustrated in Figure 4.1a. Note that pointstabilization is a special case of trajectory tracking where the nominal trajectory collapses to a single point $\left(x_{r}, u_{r}\right)$.

In path following, the obtained reference from the motion planner consists of nominal vehicle states $x_{r}(s)$ and control inputs $u_{r}(s)$ that are parametrized in space rather than in time. In other words, the path-following controller acts upon a space-parametrized path-following error that is implicitly parametrized in time. Given the vehicle's current state $x(t)$, the nominal vehicle state $x_{r}(s(t))$ and control input $u_{r}(s(t))$ are calculated by projecting the position $p(t)$ of the vehicle onto the nominal path in $p_{r}(s), s \in[0, \infty]$, as illustrated in Figure 4.1b.

For wheeled vehicles, the control signals typically consist of the steering angle $\alpha(t)$ and the longitudinal velocity $v_{1}(t)$ of the vehicle. The path-following controller uses the steering angle to stabilize the vehicle around the nominal path and

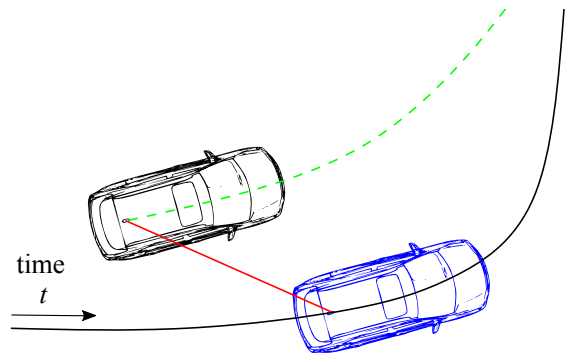

(a)

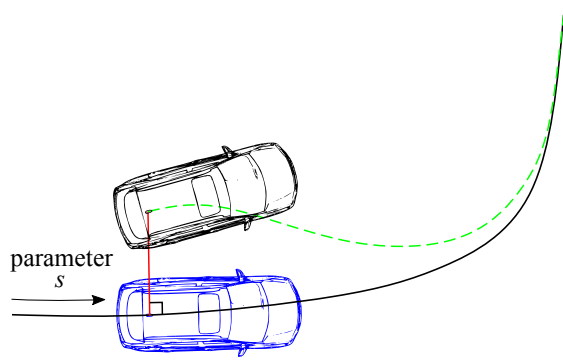

(b)

Figure 4.1: Illustration of the difference between trajectory tracking (a) and path following (b). The black vehicle is the controlled vehicle, the blue vehicle represents the reference vehicle and the red line is the positional control error. The green line represents a typical convergence behavior of the closed-loop system when trajectory-tracking control (a) and path-following control (b) is used, respectively. 
the longitudinal velocity is controlled such that the nominal path is executed at a desired set speed $v_{r}(t)$. In this setup, the control task is said to be separated into lateral and longitudinal control. Figure 4.1b illustrates how a path-following controller operates to stabilize the vehicle around the nominal path. For small control errors, a trajectory-tracking controller and a path-following controller performs similarly. However, if a large longitudinal tracking error is present in a sharp corner, a trajectory-tracking controller can behave undesirably and take shortcuts since longitudinal and lateral control are coupled.

\subsection{Trajectory-tracking control}

In trajectory-tracking control applications, the motion planner is assumed to provide the feedback controller with a collision-free nominal trajectory ${ }^{2}\left(x_{r}(t), u_{r}(t)\right)$, $t \in[0, \infty)$. Since the nominal trajectory is feasible, it satisfies the vehicle model

$$
\dot{x}_{r}(t)=f\left(x_{r}(t), u_{r}(t)\right), \quad x_{r}(0) \text { given, }
$$

and the vehicle's physically imposed constraints $x_{r}(t) \in \mathbb{X}$ and $u_{r}(t) \in \mathbb{U}, \forall t \geq 0$. The objective of the trajectory-tracking controller is to control the vehicle's state trajectory $x(\cdot)$ such that the nominal trajectory (4.1) is executed with a small tracking error $\bar{x}(t)=x(t)-x_{r}(t)$. Denote the control-signal deviation as $\bar{u}(t)=$ $u(t)-u_{r}(t)$. Then the tracking-error system becomes

$$
\dot{\bar{x}}(t)=f\left(\bar{x}(t)+x_{r}(t), \bar{u}(t)+u_{r}(t)\right)-f\left(x_{r}(t), u_{r}(t)\right) \triangleq \bar{f}(t, \bar{x}(t), \bar{u}(t)),
$$

where $(\bar{x}(t), \bar{u}(t))=(0,0)$ is an equilibrium point since $\bar{f}(t, 0,0)=0, \forall t \geq 0$ and the initial tracking error $\bar{x}(0)=x(0)-x_{r}(0)=\bar{x}_{0}$ is given. Note that the nominal trajectory $\left(x_{r}(\cdot), u_{r}(\cdot)\right)$ makes the tracking-error system in (4.2) become timevarying. Define the feedback controller as $\bar{u}(t)=g(t, \bar{x}(t))$ where $g(t, 0)=0$. With this feedback control-law, the closed-loop tracking-error system can compactly be written as

$$
\dot{\bar{x}}(t)=\bar{f}(t, \bar{x}, g(t, \bar{x}(t))) \triangleq \bar{f}_{c l}(t, \bar{x}(t)), \quad \bar{x}(0)=\bar{x}_{0},
$$

where the origin $\bar{x}(t)=0$ is an equilibrium point since $f_{c l}(t, 0)=\bar{f}(t, 0,0)=0, \forall t \geq 0$. The closed-loop tracking-error system in (4.3) is a nonlinear time-varying system and the objective is to design the trajectory-tracking controller $\bar{u}(t)=g(t, \bar{x}(t))$ such that the origin of (4.3) obtains some of the following properties.

Definition 4.1 (Khalil and Grizzle (2002) Definition 4.4). The equilibrium point $\bar{x}=0$ of $(4.3)$ is

- Stable, if, for each $\epsilon>0$, there is a $\delta=\delta\left(\epsilon, t_{0}\right)>0$ such that

$$
\left\|\bar{x}\left(t_{0}\right)\right\|<\delta \Rightarrow\|\bar{x}(t)\|<\epsilon, \quad \forall t \geq t_{0} \geq 0 .
$$

\footnotetext{
${ }^{2}$ In the previous chapter, it was assumed that the motion plan is constructed such that the goal is an equilibrium point, i.e., $f\left(x_{r}(t), u_{r}(t)\right)=0, t \geq t_{G}$. This assumption implies that the motion plan can be extended to infinity where the vehicle is specified to remain it the goal state $\forall t \geq t_{G}$.
} 
- Uniformly stable if, for each $\epsilon>0$, there is a $\delta=\delta(\epsilon)>0$, independent of $t_{0}$, such that (4.4) is satisfied.

- Asymptotically stable if it is stable and there is a positive constant $c=c\left(t_{0}\right)$ such that $\bar{x}(t) \rightarrow 0$ as $t \rightarrow \infty$, for all $\left\|\bar{x}\left(t_{0}\right)\right\|<c$.

Other desirable stability properties of the origin includes exponential stability where the rate of convergence is upper-bounded by an exponential decay and uniform asymptotic stability which means that asymptotic stability around the origin is independent of the initial time (Khalil and Grizzle, 2002). In many applications, global stability properties of the origin for the closed-loop trackingerror system (4.3) are hard to establish and only local stability properties can be verified (Paden et al., 2016).

Generally, the problem of determining uniform stability (uniform asymptotic stability) of the closed-loop system in (4.3) is to find a Lyapunov function candidate $V(t, \bar{x}) \geq 0$, where its time derivative

$$
\dot{V}(t, \bar{x})=\frac{\partial V(t, \bar{x})}{\partial \bar{x}} \bar{f}_{c l}(t, \bar{x}(t))+\frac{\partial V(t, \bar{x})}{\partial t},
$$

is non-positive (negative) for all $t \geq 0$ in a neighborhood around the origin (Khalil and Grizzle, 2002). For more details regarding stability of nonlinear systems, the reader is referred to (Khalil and Grizzle, 2002). Some techniques are now presented for designing the trajectory-tracking controller $\bar{u}(t)=g(t, \bar{x}(t))$.

\subsubsection{Linear trajectory-tracking techniques}

Linear control techniques is a simple but yet efficient design tool for trajectory tracking of nonlinear systems where the nonlinear tracking-error system (4.2) is first linearized around the nominal trajectory. Since the nominal trajectory is feasible, it satisfies the vehicle model and the vehicle's physically imposed constraints $x_{r}(t) \in \mathbb{X}$ and $u_{r}(t) \in \mathbb{U}, \forall t \geq 0$. Typically, the constraints are assumed to be satisfied with a certain margin, making it possible for the trajectory-tracking controller to suppress disturbances during the execution of the nominal trajectory. Then, depending on the application, it can sometimes be reasonable to neglect the constraints during the control design if the initial tracking error $\bar{x}(0)$ is small and the designed controller is able to locally stabilize the vehicle around the nominal trajectory. A first-order Taylor series expansion of the tracking-error system (4.2) around the nominal trajectory yields

$$
\dot{\bar{x}}(t)=A(t) \bar{x}(t)+B(t) \bar{u}(t), \quad \bar{x}(0)=\bar{x}_{0},
$$

where

$$
\begin{aligned}
& A(t)=A\left(x_{r}(t), u_{r}(t)\right)=\left.\frac{\partial f(x(t), u(t))}{\partial x}\right|_{\left(x_{r}(t), u_{r}(t)\right)}, \\
& B(t)=B\left(x_{r}(t), u_{r}(t)\right)=\left.\frac{\partial f(x(t), u(t))}{\partial u}\right|_{\left(x_{r}(t), u_{r}(t)\right)} .
\end{aligned}
$$


The system in (4.6) is a linear time-varying (LTV) system. Here, we are interested in stabilizing this system around the origin using a linear state-feedback controller with feedforward action

$$
u(t)=u_{r}(t)+K(t) \bar{x}(t)
$$

where $K(t) \in \mathbb{R}^{m \times n}$ is the state-feedback gain. Note that the feedforward $u_{r}(t)$ is provided by the motion planner and therefore included in (4.8). With this statefeedback controller inserted in the nonlinear tracking-error system (4.2), the closedloop system is on the form in (4.3). The objective is now to design the feedback gain $K(t)$ such that the origin to this closed-loop system obtains desired stability properties. By inserting the control-law in (4.8) into the linearized tracking-error system (4.6), the closed-loop system becomes

$$
\dot{\bar{x}}(t)=(A(t)+B(t) K(t)) \bar{x}(t) \triangleq A_{c l}(t) \bar{x}(t), \quad \bar{x}(0)=\bar{x}_{0} .
$$

Global exponential stability of the origin for this system follows from Theorem 4.1.

Theorem 4.1 (Khalil and Grizzle (2002)). Let $A_{c l}(t)$ be continuous $\forall t \geq 0$. Suppose there exists a continuously differentiable $P(t) \succ 0$ that satisfies

$$
0 \prec c_{1} I \preceq P(t) \preceq c_{2} I, \quad \forall t \geq 0,
$$

for positive constants $c_{1}$ and $c_{2}$, where $c_{1}<c_{2}$. If $P(t)$ satisfies the Lyapunov inequality

$$
\dot{P}(t)+P(t) A_{c l}(t)+A_{c l}^{\top}(t) P(t) \preceq-Q, \quad \forall t \geq 0,
$$

with $Q \succ 0$, then, the origin to (4.9) is a globally exponentially stable equilibrium point.

Proof: See proof of Theorem 4.10 in Khalil and Grizzle (2002).

The condition in (4.10) is equivalent to the existence of a Lyapunov function $V(t, \bar{x})=\bar{x}^{\top} P(t) \bar{x}$ that satisfies $\dot{V}(t, \bar{x}) \leq-\bar{x}^{\top} Q \bar{x}, \forall t \geq 0$, for the closed-loop LTV system in (4.9). In fact, exponential stability of the origin of the closed-loop LTV system in (4.9) is a sufficient condition for exponential stability of the origin of the nonlinear closed-loop system in (4.3). The result is formalized in Theorem 4.2.

Theorem 4.2 (Khalil and Grizzle (2002)). Let $\bar{x}(t)=0$ be an equilibrium point for the nonlinear system in (4.3) where $\tilde{f}_{c l}:[0, \infty) \times D \rightarrow \mathbb{R}^{n}$ is continuously differentiable, $D=\left\{\bar{x} \in \mathbb{R}^{n} \mid\|\bar{x}\|_{2}<r\right\}$, and the Jacobian matrix $A_{c l}(t)$ is bounded and Lipschitz on $D$, uniformly in $t \geq 0$. Then, the origin is an exponentially stable equilibrium point for the nonlinear system in (4.3) if it is an exponentially stable equilibrium point for the LTV system in (4.9).

Proof: See proof of Theorem 4.15 in Khalil and Grizzle (2002).

Remark 4.1. Both Theorem 4.1 and Theorem 4.2 have a finite-time counter part. In that case, exponential stability is replaced with the norm of the tracking error $\|\bar{x}(t)\|$ being upper bounded by a positive and exponentially decreasing function in time. 
To conclude, if $K(t)$ is designed such that the closed-loop LTV system in (4.9) satisfies Theorem 4.1. Then, Theorem 4.2 ensures exponential stability of the origin for the nonlinear closed-loop system in (4.3). Now, some methods are presented for designing the feedback gain $K(t)$ such that Theorem 4.1 is satisfied.

\subsubsection{Robust control design using linear matrix inequalities}

In some applications, a static state-feedback gain $K$ is enough to stabilize the LTV system in (4.9). One way to make use of Theorem 4.1 is to assume that the pairs $[A(t), B(t)]$ lie in the convex polytope $\mathbb{P}, \forall t \geq 0$, where $\mathbb{P}$ is represented by $L$ vertices

$$
[A(t), B(t)] \in \mathbb{P}=\mathbf{C o}\left\{\left[A_{1}, B_{1}\right], \ldots,\left[A_{L}, B_{L}\right]\right\},
$$

where Co denotes the convex hull. Denote the polytopic linear differential inclusion (LDI) (Boyd et al., 1994) as

$$
\dot{\bar{x}}(t) \in \mathbb{P}\left[\begin{array}{l}
\bar{x}(t) \\
\bar{u}(t)
\end{array}\right], \quad \bar{x}(0)=\bar{x}_{0} .
$$

We can interpret the polytopic LDI in (4.12) as describing a family of LTV systems including (4.6). This implies that a solution to (4.6) is also a trajectory of the LDI (4.12). Thus, with $\bar{u}(t)=K \bar{x}(t)$ in (4.12), global exponential stability of the closed-loop version of (4.12) implies global exponential stability of the closedloop LTV system in (4.9). With a constant Lyapunov matrix $P(t)=P \succ 0$ in Theorem 4.1, the condition (4.10) for global exponential stability can be reformulated as a controller synthesis problem (Boyd et al., 1994) in the form of a matrix inequality feasibility problem in the variables $P \succ 0$ and $K \in \mathbb{R}^{m \times n}$

$$
\left(A_{i}+B_{i} K\right)^{\top} P+P\left(A_{i}+B_{i} K\right) \preceq-2 \epsilon P, \quad i=1, \ldots, L,
$$

where $\epsilon>0$ is a constant and $Q \succ 0$ in (4.10) has been replaced with $2 \epsilon P \succ 0$. Here, $\epsilon>0$ denotes the decay-rate of the Lyapunov function (Boyd et al., 1994). This matrix inequality is not jointly convex in $P$ and $K$. However, if $\epsilon>0$ is fixed, with the bijective transformation $S=P^{-1} \succ 0$ and $Y=K P^{-1} \in \mathbb{R}^{m \times n}$, the matrix inequality in (4.13) can be rewritten as a linear matrix inequality (LMI) in $S$ and $Y$ (Wolkowicz et al., 2012):

$$
S A_{i}^{\top}+Y^{\top} B_{i}^{\top}+A_{i} S+B_{i} Y+2 \epsilon S \preceq 0, \quad i=1, \ldots, L .
$$

In other words, it is an LMI feasibility problem to find a linear state-feedback controller $\bar{u}(t)=K \bar{x}(t)$ that satisfies condition (4.10) in Theorem 4.1. If $S$ and $Y$ are feasible solutions to (4.14), the quadratic Lyapunov function is $V(\bar{x}(t))=$ $\bar{x}(t)^{\top} S^{-1} \bar{x}(t)$ and the linear state-feedback controller is $\bar{u}(t)=Y S^{-1} \bar{x}(t)$. Moreover, by Theorem 4.2, the origin of the nonlinear closed-loop system in (4.3) is an exponentially stable equilibrium point. The constraint in (4.14) can be used in a semi-definite programming (SDP) problem by, e.g., minimizing $\left\|K-K_{\text {des }}\right\|$, where $K_{\text {des }}$ is a nominal feedback gain. Another possibility is to minimize the condition number of $S^{-1}$ (Boyd et al., 1994). 
If a single static state-feedback controller is not enough to stabilize the vehicle around the nominal trajectory, gain-scheduling can be used (Rugh and Shamma, 2000). Here, a set of working points is selected and a state-feedback gain $K\left(x_{r}, u_{r}\right)$ is designed for each working point $\left(x_{r}, u_{r}\right)$ (Rugh and Shamma, 2000). The resulting gain-scheduled linear feedback controller with feedforward action is

$$
u(t)=u_{r}(t)+K\left(x_{r}(t), u_{r}(t)\right) \bar{x}(t),
$$

where the state-feedback gain is typically obtained using a convex-combination of neighboring working points to $\left(x_{r}(t), u_{r}(t)\right)$. Unless the matrices $A\left(x_{r}(t), u_{r}(t)\right)$ and $B\left(x_{r}(t), u_{r}(t)\right)$ are slowly-varying, stability for the resulting closed-loop LTVsystem cannot be formally guaranteed (Rugh and Shamma, 2000). Thus, extensive simulations and experiments need to be conducted to validate a gain-scheduled controller. This is however the case for any feedback-control strategy in practice.

\subsubsection{Linear quadratic control}

In this section, we turn to the problem of designing the linear state-feedback controller $\bar{u}(t)=K(t) \bar{x}(t)$ in an optimal way. One possibility is to use linear quadratic (LQ) control techniques. The time-varying finite-horizon LQ control problem is (Anderson and Moore, 2007):

$$
\begin{array}{cl}
\underset{\bar{u}(\cdot)}{\operatorname{minimize}} & \left.\|\bar{x}(T)\|_{P_{N}}^{2}+\int_{0}^{T}\left(\|\bar{x}(t)\|_{Q(t)}^{2}+\| \bar{u}(t)\right) \|_{R(t)}^{2}\right) \mathrm{d} t \\
\text { subject to } & \dot{\bar{x}}(t)=A(t) \bar{x}(t)+B(t) \bar{u}(t), \\
& \bar{x}(0)=\bar{x}_{0} \text { given, }
\end{array}
$$

where $T>0$ is the prediction horizon, $P_{N} \succeq 0$ is the terminal cost, $R(t) \succ 0$ and $Q(t) \succeq 0$ are design matrices that are used to trade-off between large tracking errors and control-signal deviations. Here, $A(t)$ and $B(t)$ describe the time-varying linearization (4.6) of the nonlinear tracking-error system in (4.2) around the nominal trajectory $\left(x_{r}(\cdot), u_{r}(\cdot)\right)$. Since $R(t) \succ 0$, the Hamiltion-Jacobi-Bellman (HJB) equation (Anderson and Moore, 2007) is strictly convex in $\bar{u}(t)$ and the optimal solution to the finite-horizon LQ control problem is a linear state-feedback control law $\bar{u}(t)=K(t) \bar{x}(t)$ where the time-varying feedback gain is

$$
K(t)=-R^{-1}(t) B^{\top}(t) P(t),
$$

and $P(t)=P^{\top}(t)$ is obtained by solving a matrix Riccati partial differential equation

$$
-\dot{P}(t)=A^{\top}(t) P(t)+P(t) A(t)-P(t) B(t) R^{-1} B^{\top}(t) P(t)+Q(t),
$$

with boundary condition $P(T)=P_{N}$ (Kalman et al., 1960). Furthermore, the optimal cost for the finite-horizon LQ control problem is $V(t, \bar{x})=\bar{x}^{\top}(t) P(t) \bar{x}(t)$. In general, an explicit solution to (4.18) does not exists but it can be computed 
numerically (Anderson and Moore, 2007; Kalman et al., 1960; Zhou et al., 1996). The discrete-time version of (4.16) instead yields a solution $P\left(t_{k}\right)$ that satisfies a matrix Riccati recursion (Anderson and Moore, 2007; Axehill, 2005; Nielsen, 2017).

The feedback controller defined in (4.17) and (4.18) can be deployed in practice using a receding horizon control strategy where the problem is solved over a moving time window. Here, a matrix Riccati partial differential equation (4.18), or a matrix Riccati recursion for the discrete-time case (Anderson and Moore, 2007; Axehill, 2005), has to be computed at each sampling instance.

If the matrices $A\left(x_{r}(t), u_{r}(t)\right)$ and $B\left(x_{r}(t), u_{r}(t)\right)$ are almost constant matrices, one single operating point $\left(x_{r}, u_{r}\right)$ can be used to design the feedback gain. This motivates treating the LTV system as a linear time-invariant (LTI) system given by the constant system matrices $A=A\left(x_{r}, u_{r}\right)$ and $B=B\left(x_{r}, u_{r}\right)$. The timeinvariant infinite-horizon LQ control problem is

$$
\begin{aligned}
\underset{\bar{u}(\cdot)}{\operatorname{minimize}} & \left.\int_{0}^{\infty}\left(\|\bar{x}(t)\|_{Q}^{2}+\| \bar{u}(t)\right) \|_{R}^{2}\right) \mathrm{d} t \\
\text { subject to } & \dot{\bar{x}}(t)=A \bar{x}(t)+B \bar{u}(t), \\
& \bar{x}(0)=\bar{x}_{0} \text { given. }
\end{aligned}
$$

Given that the pair $(A, B)$ is controllable and $\left(A, Q^{1 / 2}\right)$ is observable, this problem has an explicit solution and the optimal state-feedback controller is $\bar{u}(t)=K \bar{x}(t)$ where the feedback gain is

$$
K=-R^{-1} B^{\top} P,
$$

and $P \succ 0$ is the unique solution the algebraic Riccati equation (ARE)

$$
A^{\top} P+P A-P B R^{-1} B^{\top} P+Q=0 .
$$

The optimal cost for (4.19) is $V(\bar{x}(t))=\bar{x}^{\top}(t) P \bar{x}(t)$ and the resulting closed-loop system $\dot{\bar{x}}=(A+B K) \bar{x}$ is exponentially stable (Anderson and Moore, 2007) where $V(\bar{x}(t))$ is a valid Lyapunov function candidate. Note that the solution $P$ in (4.21) is often used as terminal cost $P_{N}$ for the finite-horizon LQ control problem in (4.16) where a working point $\left(x_{r}, u_{r}\right)$ is selected for its derivation (Anderson and Moore, 2007). The location of the poles to the closed-loop system are the negative eigenvalues to the Hamiltonian matrix (Anderson and Moore, 2007):

$$
H=\left[\begin{array}{cc}
A & -B R^{-1} B^{\top} \\
-Q & -A^{\top}
\end{array}\right] .
$$

The location of these poles provides information about the system's time constants and thus the required length of the prediction horizon $T$ to obtain an good tradeoff between tracking performance and computational complexity. Infinite-horizon LQ control is a popular control strategy due to its simplicity, robustness margins and stability guarantees (Anderson and Moore, 2007). However, if an observer is used to estimate the vehicle states needed for control, all guarantees are lost (Doyle, 
1978). The solution to the LQ problem is optimal in the sense that it minimizes the specified cost function given that the model is correct. However, in practice model uncertainties and other disturbances are always present. $H_{\infty}$ control (Helmersson, 1995; Ravi et al., 1991; Zhou et al., 1996) is a structured design approach for taking uncertainties and disturbances into account while synthesizing the controller. $H_{\infty}$ control has been proposed for robust lateral control of different tractor-trailer combinations in, e.g., Kati et al. (2016, 2019a,b); Tai et al. (1999); Varga et al. (2018) to account for, e.g., uncertain moments of inertia and varying road-surface conditions during onroad driving.

\subsubsection{Model predictive control}

One of the most powerful tools for designing the feedback controller is to deploy online optimization techniques where an OCP is solved online at a specified sampling rate. For the previously presented control strategies, the physically imposed constraints on the vehicle states $x(t) \in \mathbb{X}$ and control input $u(t) \in \mathbb{U}$ are hard to take into account for during the control design. This can be motivated during moderate driving conditions where the nominal trajectory is feasible. However, if the motion planner uses a too simplistic model of the vehicle or if large disturbances are present, it is not certain that the actual motion plan is feasible to follow. This can for example be the case during slippery road surface conditions or during emergency maneuvers where vehicle models with higher fidelity have to be used (Rajamani, 2011). To handle such situations, more responsibility is commonly laid on the feedback controller to take care of both local replanning and feedback control (Paden et al., 2016; Svensson et al., 2019).

Model predictive control (MPC) (Garcia et al., 1989) is a powerful control strategy which can be very efficient for such scenarios. Similar to the finite-horizon LQ controller (4.16), an MPC controller uses a model of the vehicle to predict and optimize the vehicle's future state and control-input trajectories. Here, a nonlinear model of the vehicle could be used and the vehicle's physical constraints can be taken into account during the control design.

To obtain feedback properties, the idea with MPC is to only use the first portion of the control input and instead repetitively solve a receding horizon OCP at every time step $\Delta t$. Given the current vehicle state $x(t)$, the MPC control law $u(t)$ is computed by solving the continuous-time nonlinear MPC problem

$$
\begin{aligned}
\underset{u(\cdot)}{\operatorname{minimize}} & \left.\Phi_{T}(\bar{x}(t+T))+\int_{t}^{t+T}\left(\left\|\left.\bar{x}(\tau)\right|_{Q} ^{2}+\right\| \bar{u}(\tau)\right)||_{R}^{2}\right) \mathrm{d} \tau \\
\text { subject to } & \frac{\mathrm{d} x}{\mathrm{~d} \tau}=f(x(\tau), u(\tau)), \\
& u(\tau) \in \mathbb{U}, \quad x(\tau) \in \mathbb{X} \\
& x(t) \text { given. }
\end{aligned}
$$

Here, $T$ denotes the prediction horizon, $\Phi_{T}(\bar{x}(t+T)) \geq 0$ is the terminal cost, and $Q \succeq 0$ and $R \succ 0$ are design matrices. The terminal cost is often chosen 
as $\Phi_{T}(\bar{x}(t+T))=\|\bar{x}(t+T)\|_{P}^{2}$, where $P \succeq 0$ is a design matrix. The matrix $P$ is often selected as the solution to a continuous-time algebraic Riccati equation (4.21) where the nonlinear system is linearized around a working point $\left(x_{r}, u_{r}\right)$, e.g., a straight reference trajectory for a wheeled vehicle. The idea is that $\| \bar{x}(t+T)||_{P}^{2}$ is used to approximate the remaining cost-to-go after the prediction horizon and thus emulate the infinite-horizon cost. An appropriate design of the terminal cost is especially important when the selection of $T$ is small compared to the vehicle's time constants.

In contrast to the motion planning problem in (3.2), the obstacle imposed constraints are often neglected at the trajectory-tracking level and only the vehicle's physical limitations are considered. Instead, a quadratic penalty on the deviation from the nominal trajectory $\left(x_{r}(\cdot), u_{r}(\cdot)\right)$ is used as performance measure.

To solve the continuous-time OCP in (4.23), it is first discretized to obtain an NLP. Since the vehicle model in general is nonlinear, the resulting NLP problem is non-convex and thus hard to solve to optimality. However, efficient method exists for real-time deployment of approximate solution to the resulting NLP problem. One example is linear MPC, where the NLP is simplified to a quadratic programing (QP) problem, e.g., by linearizing the nonlinear vehicle model around the nominal trajectory or path. Another example is real-time iterations (RTI) (Gros et al., 2020; Houska et al., 2011a) where instead of solving the discretized version of (4.23) to local optimality, only a few (commonly one) SQP iterations are performed at each sampling instance. The idea is that if several sampling instances are considered, an RTI scheme is basically resembling an SQP method. However, in RTI the current vehicle state can be updated as new information arrives and the preliminary solution can be used as control signal before the solver has completely converged. One example of open source software for RTI is the ACADO code generation toolkit (Houska et al., 2011a) which can be used to automatically generate C-code for efficient approximate nonlinear MPC. The generated code solves a sequence of quadratic programs using qpOASES (Ferreau et al., 2014) which is a parametric active-set solver with warm starts.

Similar to $H_{\infty}$ control (Zhou et al., 1996), robust MPC (Löfberg, 2003; Paden et al., 2016; Pereira et al., 2018) is a robust control strategy to take model uncertainties and disturbances into account while designing the MPC controller.

Trajectory-tracking control using MPC was first utilized in the process industry to control slowly-varying systems (Rawlings, 2000). Advances in computing power as well as mathematical programming algorithms have made MPC feasible for real-time deployment in self-driving vehicles. Surveys of existing work for car-like vehicles can be found in, e.g., Lima (2018); Paden et al. (2016).

\subsubsection{Trajectory-tracking control using LQ control: An example}

A trajectory-tracking example for a kinematic bicycle model (2.11) is now presented. The state vector is $x(t)=\left[\begin{array}{lll}x_{1}(t) & y_{1}(t) & \theta_{1}(t)\end{array}\right]^{\top}$, where $\left(x_{1}, y_{1}\right)$ is the position for the center of the vehicle's rear axle and $\theta_{1}$ is its orientation, see Figure 4.2. The control-input vector is $u(t)=\left[v_{1}(t) \kappa(t)\right]^{\top}$ where $v_{1}$ is the longitudinal velocity for the vehicle's rear axle and $\kappa$ is its curvature. Assume a nominal trajectory 


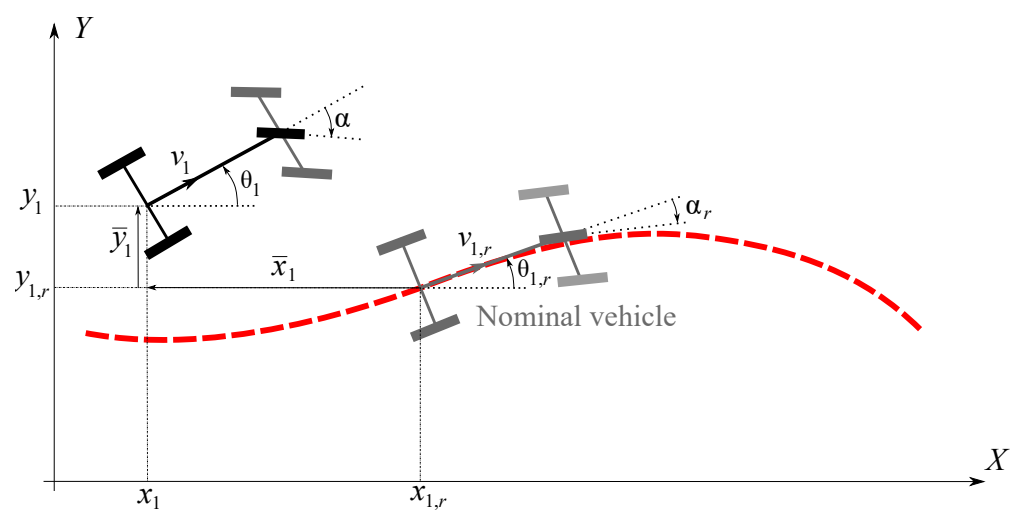

Figure 4.2: The kinematic bicycle model at state $x(t)$ and the nominal vehicle at state $x_{r}(t)$.

$\dot{x}_{r}(t)=f\left(x_{r}(t), u_{r}(t)\right)$ is provided by a motion planner. Denote the tracking error as $\bar{x}(t)=x(t)-x_{r}(t)$ and the control-input deviation as $\bar{u}(t)=u(t)-u_{r}(t)$. Then, the tracking-error model can compactly be written as $\dot{\bar{x}}(t)=f_{c l}(t, \bar{x}(t), \bar{u}(t))$. Since the model is defined in a global coordinate system, it is not possible to design a static linear state-feedback controller $u(t)=u_{r}(t)+K \bar{x}(t)$ which stabilizes the tracking-error system unless a coordinate transformation, similar to Kanayama et al. (1990), is first performed. By the following change of basis

$$
\left[\begin{array}{l}
x_{1, e}(t) \\
y_{1, e}(t) \\
\theta_{1, e}(t)
\end{array}\right]=T(t, \bar{x}(t)) \bar{x}(t)=\left[\begin{array}{ccc}
\cos \theta_{1}(t) & \sin \theta_{1}(t) & 0 \\
-\sin \theta_{1}(t) & \cos \theta_{1}(t) & 0 \\
0 & 0 & 1
\end{array}\right]\left[\begin{array}{c}
\bar{x}_{1}(t) \\
\bar{y}_{1}(t) \\
\bar{\theta}_{1}(t)
\end{array}\right],
$$

the tracking-error system in the new coordinate frame is (see Appendix A)

$$
\begin{aligned}
& \dot{x}_{1, e}(t)=y_{1, e}(t) v_{1}(t) \kappa(t)+v_{1}(t)-v_{1, r}(t) \cos \theta_{1, e}(t), \\
& \dot{y}_{1, e}(t)=-x_{1, e}(t) v_{1}(t) \kappa(t)+v_{1, r}(t) \sin \theta_{1, e}(t), \\
& \dot{\theta}_{1, e}(t)=v_{1}(t) \kappa(t)-v_{1, r}(t) \kappa_{r}(t) .
\end{aligned}
$$

Define $x_{e}=\left[\begin{array}{lll}x_{1, e} & y_{1, e} & \theta_{1, e}\end{array}\right]^{\top}$ and represent the tracking-error model in (4.25) as $\dot{x}_{e}=f_{e}\left(t, x_{e}, \bar{u}\right)$. Clearly, $x_{e}(t)=0$ if and only if $\bar{x}(t)=0$ because $T(t, \bar{x})$ is nonsingular $(\operatorname{det} T(t, \bar{x})=1)$. Since the origin $\left(x_{e}, \bar{u}\right)=(0,0)$ to $(4.25)$ is an equilibrium point, a first-order Taylor-series expansion around the origin yields

$$
\dot{x}_{e}=\underbrace{\left[\begin{array}{ccc}
0 & v_{1, r}(t) \kappa_{r}(t) & 0 \\
-v_{1, r}(t) \kappa_{r}(t) & 0 & v_{1, r}(t) \\
0 & 0 & 0
\end{array}\right]}_{\triangleq A(t)} x_{e}(t)+\underbrace{\left[\begin{array}{cc}
1 & 0 \\
0 & 0 \\
\kappa_{r}(t) & v_{1, r}(t)
\end{array}\right]}_{\triangleq B(t)} \bar{u}(t) .
$$

This system is an LTV system with time-varying matrices $A(t)$ and $B(t)$ that depend on the nominal velocity $v_{1, r}(t)$ and nominal curvature $\kappa_{r}(t)$ trajectories. This system can also be viewed as a linear parameter-varying (LPV) system where 


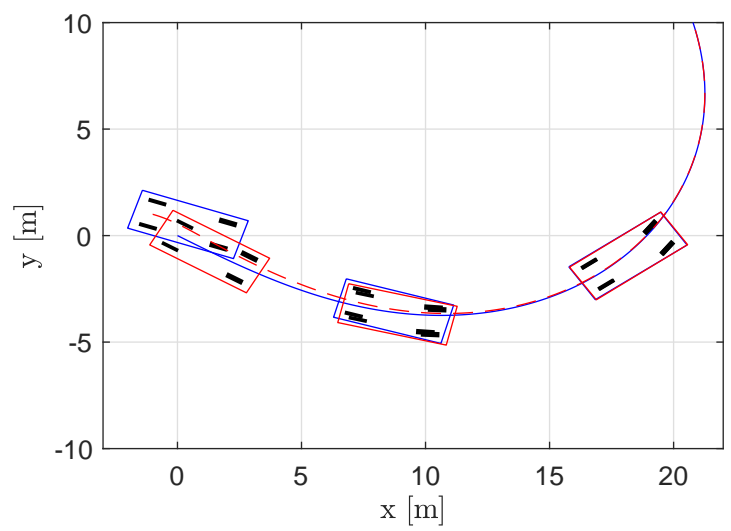

Figure 4.3: Trajectory tracking for a kinematic bicycle model with initial tracking error $x_{e}(0)=\left[\begin{array}{lllll}-1 & \mathrm{~m} & 1 & \mathrm{~m} & 0.2 \mathrm{rad}\end{array}\right]^{\top}$. The controlled vehicle is plotted in blue and the nominal vehicle in red. The vehicles are plotted at $t=0,7$ and $14 \mathrm{~s}$. The red dotted path displays the trajectory for the controlled vehicle's position and the blue path is its nominal trajectory.

$v_{1, r}(t)$ and $\kappa_{r}(t)$ are the time-varying parameters. As long as $v_{1, r} \neq 0$, the system is controllable but it can be difficult to stabilize the vehicle over a wide range of nominal curvature and velocity profiles. Some approaches to handle this include finite-horizon LQ control (4.16) or gain-scheduling techniques (4.15). As a simple example, a single infinite-horizon LQ controller (4.19) is designed around the working point $\left(v_{1, r}, \kappa_{r}\right)=(1,0)$. With the weight matrices $Q=\operatorname{diag}([1,1,10])$ and $R=\operatorname{diag}([10,40])$, the LQ controller is given by

$$
u(t)=u_{r}(t)+\underbrace{\left[\begin{array}{ccc}
-0.3162 & 0 & 0 \\
0 & -0.1581 & -0.7525
\end{array}\right]}_{\triangleq K} x_{e}(t) .
$$

From the structure of $K$ it is clear that the longitudinal error $x_{1, e}$ is controlled by $v_{1}(t)$ and the lateral $y_{1, e}$ and angular $\theta_{1, e}$ errors are controlled by $\kappa(t)$. This is, however, not the case if the system is linearized around a working point $\kappa_{r} \neq 0$. To evaluate the controller's performance, a nominal trajectory is generated by simulating the vehicle using a sinusoidal velocity and curvature profile

$$
\begin{aligned}
v_{1, r}(t) & =1+A_{v} \sin \left(w_{v} t\right), \\
\kappa_{r}(t) & =A_{\kappa} \sin \left(w_{\kappa} t\right),
\end{aligned}
$$

with $w_{v}=w_{\kappa}=0.1 \mathrm{rad} / \mathrm{s}, A_{v}=0.5 \mathrm{~m} / \mathrm{s}$ and $A_{\kappa}=0.1 \mathrm{~m}^{-1}$. To perform more realistic simulations, additional first-order systems for the curvature and the longitudinal velocity with time constants $T_{\kappa}=0.2 \mathrm{~s}$ and $T_{v}=0.5 \mathrm{~s}$, respectively, are included. The closed-loop simulation is depicted in Figure 4.3, where an initial tracking error $x_{e}(0)=[-1 \mathrm{~m} 1 \mathrm{~m} 0.2 \mathrm{rad}]^{\top}$ is used to analyze how the feedback controller handles disturbance rejection. As can be seen from Figure 4.3, the tracking errors converge towards zero. In Figure 4.4, the control signals are plotted together with their nominal trajectories. The results illustrate that the feedback part handles disturbance rejection and the feedforward part is responsible for tracking. 


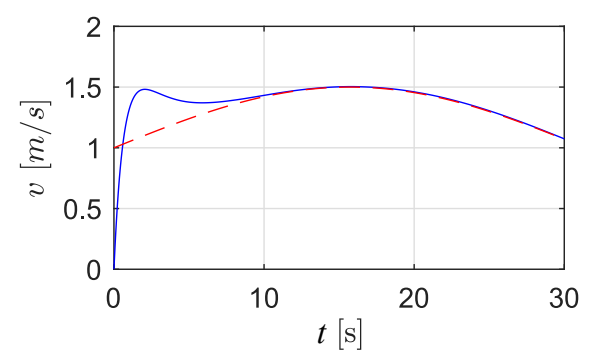

(a)

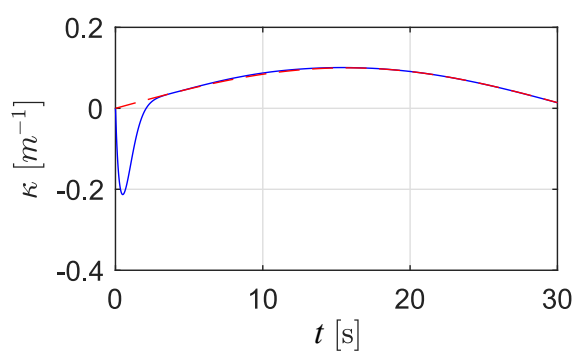

(b)

Figure 4.4: The control signals (blue line) and their feedforwards (dashed red) during the trajectory-tracking example.

\subsection{Path-following control}

In self-driving vehicle applications, it is often convenient to separate the control problem into lateral and longitudinal control (Paden et al., 2016). The lateral controller is called a path-following controller and its objective is to make sure that a nominal path $\left(x_{r}(s), u_{r}(s)\right), s \in\left[0, s_{G}\right]$ obtained from the motion planner is followed with a small and bounded path-following error. After initial transients have been eliminated, the longitudinal controller is designed such that the motion plan is executed at a desired velocity, i.e., $\dot{s}(t)=v_{1, r}(t)$. In the sequel, it is assumed that the system is a wheeled vehicle ${ }^{3}$ represented by a state vector $x(t)$ that can be partitioned as $x=\left[\begin{array}{lll}p^{\top} & \theta_{1} & \eta^{\top}\end{array}\right]^{\top}$, where $p(t)=\left(x_{1}(t), y_{1}(t)\right)$ denotes the position of the vehicle, $\theta_{1}(t)$ its orientation and $\eta(t)$ represents additional vehicle states, e.g., $N$ joint angles for the general N-trailer vehicle (2.24). Moreover, the position $p(t)$ of the vehicle is assumed to have a velocity vector $v_{1}(t)$ that is aligned with its orientation $\theta_{1}(t)$, i.e., the position of the vehicle evolves as

$$
\dot{p}(t)=v_{1}(t)\left[\begin{array}{l}
\cos \theta_{1}(t) \\
\sin \theta_{1}(t)
\end{array}\right] .
$$

Examples of systems that satisfy these assumptions are the kinematic bicycle model (2.11) and the general N-trailer vehicle (2.24).

The separation between lateral and longitudinal control is naturally performed by representing the vehicle in a path-coordinate system called the Frenet-Serret frame (Bishop, 1975). The Frenet-Serret frame is a coordinate system that moves along with the controlled vehicle. It is used to represent the position of the vehicle $p(t)$ in terms of its progression $s(t)$ along the path in $p_{r}(s)$ and its signed lateral distance $\tilde{z}(t)$ with respect to $p_{r}(s(t))$, see Figure 4.5. Here, $s(t)$ is defined as the projection of the vehicle's position $p(t)$ with respect to its nominal path $p_{r}(\cdot)$ at time $t$ :

$$
s(t)=\underset{\tau \in\left[0, s_{G}\right]}{\arg \min }\left\|p(t)-p_{r}(\tau)\right\|_{2} .
$$

\footnotetext{
${ }^{3}$ Note that path following can be generalized to the 3D-case as well (Kaminer et al., 2006; Sujit et al., 2014).
} 


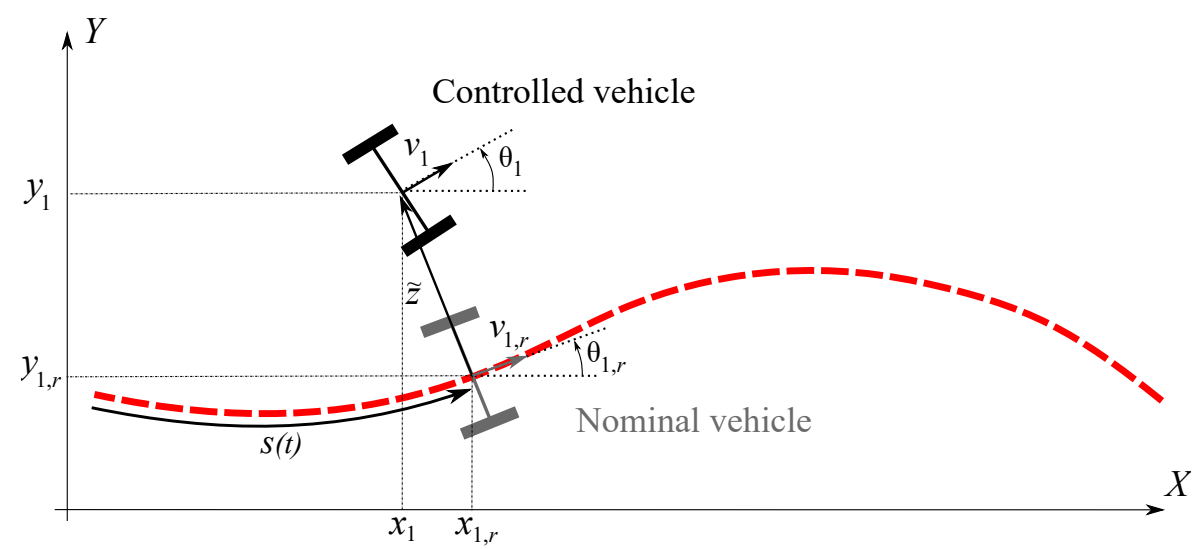

Figure 4.5: A wheel vehicle in the Frenet-Serret frame where the red-dashed line represents the nominal path in $\left(x_{1, r}(\cdot), y_{1, r}(\cdot)\right)$. The black vehicle is the controlled vehicle and the gray vehicle is the nominal vehicle at $s(t)$.

Note that at $s(t)$, there exists a nominal vehicle state $x_{r}(s(t))$ and a nominal control input $u_{r}(s(t))$. Define the curvature $\kappa_{r}(s)$ of the nominal path in $p_{r}(s)$ as

$$
\kappa_{r}(s)=\frac{d \theta_{1, r}(s)}{d s}
$$

which is assumed bounded for all $s \in\left[0, s_{G}\right]$. In the Frenet-Serret frame, the position of the vehicle $\left(x_{1}(t), y_{1}(t)\right)$ is transformed to $(s(t), z(t))$ and its model is given by (Luca et al., 1998):

$$
\begin{aligned}
& \dot{s}(t)=v_{1} \frac{\cos \tilde{\theta}_{1}}{1-\kappa_{r}(s) \tilde{z}}, \\
& \dot{\tilde{z}}(t)=v_{1} \sin \tilde{\theta}_{1},
\end{aligned}
$$

where $\tilde{\theta}_{1}(t)=\theta_{1}(t)-\theta_{1, r}(s(t))$ denotes the orientation error of the vehicle. The transformation to the Frenet-Serret frame is defined in a tube around the nominal path in $p_{r}(s)$ for which $1-\kappa_{r}(s) \tilde{z}>0$. The width of this tube depends on the nominal curvature $\kappa_{r}(s)$ and when the curvature tends to zero (a straight line) $\tilde{z}$ can vary arbitrarily. Essentially, this coordinate transformation is well-defined if $|\tilde{z}|<\left|\kappa_{r}^{-1}\right|$ when $\tilde{z}$ and $\kappa_{r}$ have the same sign. Moreover, to guarantee $\dot{s}$ to be a monotonic function in time, the orientation error $\tilde{\theta}_{1}$ is assumed to satisfy $\left|\tilde{\theta}_{1}\right|<\pi / 2$. With this assumption, $\dot{s}>0$ along forward motion segments and $\dot{s}<0$ along backward motion segments. Differentiating the orientation error $\tilde{\theta}_{1}(t)=$ $\theta_{1}(t)-\theta_{1, r}(s(t))$ with respect to time yields

$$
\dot{\tilde{\theta}}_{1}(t)=\dot{\theta}_{1}(t)+\dot{s}(t) \kappa_{r}(s(t))
$$

where the chain-rule has been applied and $\kappa_{r}(s(t))$ is defined in (4.32). The additional error states are defined as $\tilde{\eta}(t)=\eta(t)-\eta_{r}(s(t))$ and their corresponding 
differential equations $\dot{\eta}(t)$ can be obtained in analogy to (4.34). Define the pathfollowing error state vector as $\tilde{x}=\left[\begin{array}{lll}\tilde{z} & \tilde{\theta}_{1} & \tilde{\eta}^{\top}\end{array}\right]^{\top}$ and the control-input deviation as $\tilde{u}(t)=u(t)-u_{r}(s(t))$, then the wheeled vehicle can compactly be described in the path-coordinate system as

$$
\begin{aligned}
\dot{s}(t) & =f_{s}(s(t), \tilde{x}(t), \tilde{u}(t)), \\
\dot{\tilde{x}}(t) & =f_{e}(s(t), \tilde{x}(t), \tilde{u}(t)) .
\end{aligned}
$$

The origin $(\tilde{x}(t), \tilde{u}(t))=(0,0)$ to the path-following error model in $(4.35 \mathrm{~b})$ is an equilibrium point since $f_{e}(s(t), 0,0)=0$ for all $s(t) \in\left[0, s_{G}\right]$. The model in (4.35a) describes the progression of the vehicle along the nominal path, and at the origin, $\dot{s}=v_{1}(t)$.

The path-following control problem can now be formulated as follows (Paden et al., 2016): Design the feedback controller $\tilde{u}(t)=g(s(t), \tilde{x}(t))$, with $g(s(t), 0)=0$, such that the origin to the closed-loop path-following error system

$$
\dot{\tilde{x}}(t)=f_{e}(s(t), \tilde{x}(t), g(s(t), \tilde{x}(t)))=\tilde{f}_{c l}(s(t), \tilde{x}(t)),
$$

is, e.g., exponentially stable and such that the nominal path is executed at a desired set-speed $v_{1, r}(t)$. With minor adjustments, the model in (4.36) can now be stabilized using the feedback-control techniques presented in Section 4.2.

A path-following controller can easily be converted into a trajectory-tracking controller by letting the longitudinal velocity of the vehicle control the progression $s(t)$ such that a nominal trajectory in $s_{r}(t)$ is tracked. As an example, define the progression error as $\tilde{s}(t)=s(t)-s_{r}(t)$. Since $\dot{s}_{r}(t)=v_{1, r}(t)$, the longitudinal error model becomes

$$
\dot{\tilde{s}}(t)=\dot{s}(t)-\dot{s}_{r}(t)=v_{1} \frac{\cos \tilde{\theta}_{1}}{1-\kappa_{r}(s) \tilde{z}}-v_{1, r}
$$

If $v_{1}(t)$ is a control signal, the input substitution $\mu(t)=v_{1} \frac{\cos \tilde{\theta}_{1}}{1-\kappa_{r}(s) \tilde{z}}-v_{1, r}$ transforms the longitudinal error model into a single integrator system $\dot{\tilde{s}}(t)=\mu(t)$ where, e.g., a proportional-integral-derivative (PID) controller can be designed to stabilize the longitudinal error model around $\tilde{s}(t)=0$.

\subsubsection{Pure-pursuit controller}

An early proposed solution to the path-following control problem is the so-called pure-pursuit controller (Coulter, 1992; Wallace et al., 1985). This controller uses a lookahead distance $R$ to find its lookahead point $P(t)=\left(x_{p}(t), y_{p}(t)\right)$ on the reference path in $p_{r}(s)$. This point can be computed by finding the intersection between the reference path and a semi-circle with radius $R$ that is centered at the vehicle's current position $\left(x_{1}(t), y_{1}(t)\right)$, as illustrated in Figure 4.6. The pure-pursuit controller computes a desired curvature $\kappa(t)$ of the vehicle such that the lookahead point $P(t)$ is reached from the vehicle's current position $\left(x_{1}(t), y_{1}(t)\right)$. However, since the lookahead point moves along with the vehicle, a smooth motion towards the path is obtained. The pure-pursuit controller is schematically illustrated in 


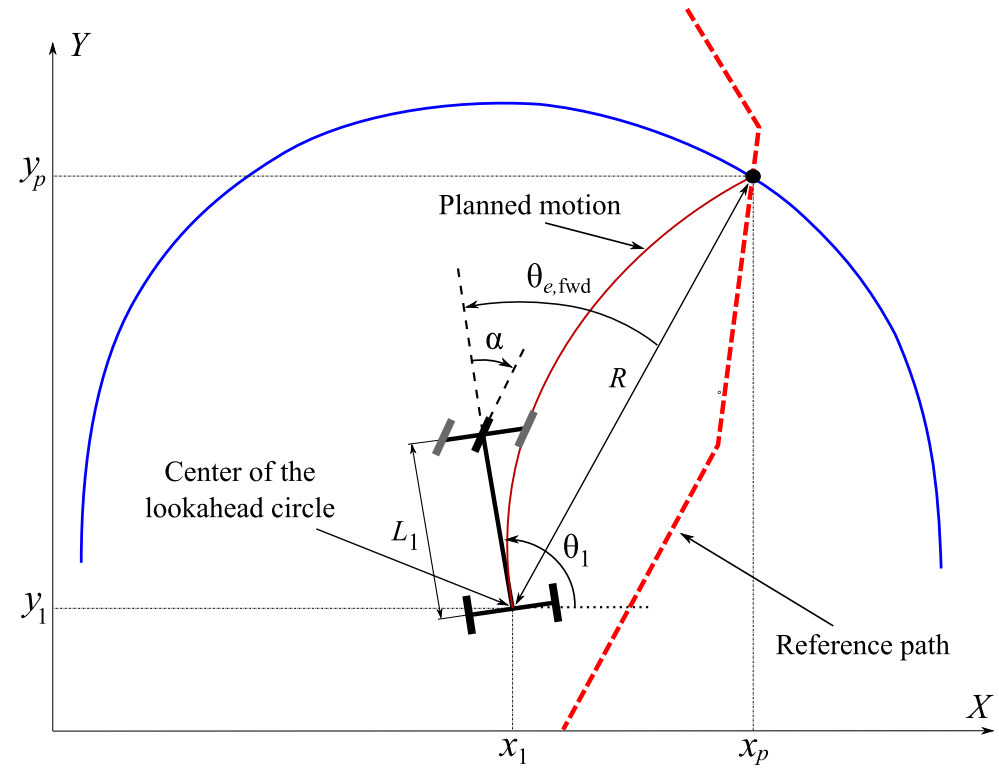

Figure 4.6: Geometric illustration of the pure-pursuit controller. The controller uses a look-ahead distance $R$ to compute the lookahead point $P$ on the path where a feedback law is derived based on the look-ahead distance and the orientation error $\theta_{e}$.

Figure 4.6 and the derivation of the feedback-law can be found in, e.g., Coulter (1992); Kuwata et al. (2009); Ljungqvist (2015). Define $\theta_{e}(t)$ as the orientation error of the vehicle with respect to the lookahead point $P(t)$. In forward motion, $\theta_{e, \mathrm{fwd}}(t)$ can be computed as

$$
\theta_{e, \mathrm{fwd}}(t)=\theta_{1}(t)-\operatorname{atan} 2\left(y_{p}(t)-y_{1}(t), x_{p}(t)-x_{1}(t)\right),
$$

where $\operatorname{atan} 2(\Delta y, \Delta x)$ denotes the generalization of $\arctan (\Delta y / \Delta x)$ over all four quadrants. Using basic geometry, the pure-pursuit feedback law in forward motion is (Kuwata et al., 2009):

$$
\kappa(t)=-\frac{2 \sin \theta_{e, \mathrm{fwd}}(t)}{R},
$$

which for the kinematic bicycle model (2.11) relates to the steering angle as $\alpha(t)=\arctan \left(L_{1} \kappa(t)\right)$. In backward motion, the semi-circle is flipped and if the orientation error $\theta_{e, \text { rev }}(t)$ is defined as follows

$$
\theta_{e, \mathrm{rev}}(t)=\operatorname{atan} 2\left(y_{1}(t)-y_{p}(t), x_{1}(t)-x_{p}(t)\right)-\theta_{1}(t)
$$

the same control-law as in (4.39) can be used in backward motion with $\theta_{e, \text { fwd }}(t)$ replaced by $\theta_{e, \text { rev }}(t)$. The design parameter for the pure-pursuit controller is the lookahead distance $R$ and the feedback law becomes less aggressive with increasing value of $R$ (Kuwata et al., 2009). However, its tracking performance degrades with increasing value of $R$. Commonly, the lookahead distance is parametrized 
with respect to the vehicle's current speed to trade-off between smoothness during high speeds (large $R$ ) and improved tracking performance during low speeds (small $R$ ) (Kuwata et al., 2009).

The pure-pursuit controller has been widely used in practice due to its simplicity and relativity satisfactory performance. For instance, two vehicles in the DARPA Grand Challenge (Buehler et al., 2007) and three vehicles in the DARPA Urban Challenge (Buehler et al., 2009) reported using a pure-pursuit controller for path-following control. One of its strengths is that the reference path does not necessarily need to be feasible to follow. However, the controller will never follow the reference path with zero path-following error unless the reference path is a straight line.

\subsubsection{Nonlinear path-following techniques}

Path-following control for nonholonomic wheeled vehicles has received a lot of attention from the automatic control and robotic communities over the last decades. A vast amount of advanced nonlinear control techniques have been proposed where the natural approach of modeling the vehicle in the Frenet-Serret frame (Bishop, 1975) is usually explored (Altafini, 2003; David and Manivannan, 2014; Fliess et al., 1995; Lima, 2018; Paden et al., 2016). In recent years, path following using MPC has started to be widely used in practice for cars (Cairano et al., 2016; Falcone et al., 2007; Plessen et al., 2018) and trucks (Lima et al., 2017a) and a survey of path-following techniques for such systems can be found in Paden et al. (2016). In Paper E and Paper G, path-following control approaches using MPC for different tractor-trailer vehicles are proposed.

For tractor-trailer vehicles with pure on-axle hitched trailers (standard Ntrailer), its flatness property can be explored to design path-following controllers using feedback linearization (Fliess et al., 1995; Rouchon et al., 1993). However, for tractor-trailer systems with a mixture of off-axle and on-axle hitching (general N-trailer), feedback linearization is not applicable (Astolfi et al., 2004; Rouchon et al., 1993). To circumvent this problem, approximate solutions have been proposed (Bolzern et al., 1998), where a simplified reference vehicle with similar stationary but different transient behavior is used for control design.

Classical path-following techniques for the general 1-trailer with a car-like tractor involve input-output linearization (Werling et al., 2014) and Lyapunov-based control design (Astolfi et al., 2004). The proposed path-following controller in Werling et al. (2014) is evaluated in field experiments for reversing a car with a trailer. Path following in forward motion for the general N-trailer using input-output linearization is proposed in Altafini (2003). The proposed solution is however limited to forward motion since the introduced zero-dynamics is unstable in backward motion.

Path-following approaches in backward and forward motion for N-trailer vehicles with pure off-axle hitched trailers (nonstandard N-trailer) using nonlinear cascade-like control methods are proposed in Michałek (2013a, 2014, 2017). In those works, the path is assumed to be represented as an analytical equation in the nominal position of the last trailer. Compared to many other path-following 
control approaches, these methods do not need to find the closest distance to the path and the complexity of the feedback controllers scales well with increasing number of trailers. By introducing artificial off-axle hitches, the proposed approaches can also be used for the GNT-case (Michałek, 2013a). However, the control approaches become sensitive to measurement noise when the distance of any signed off-axle hitch connection is close to zero.

Due to the complex kinematics of the general N-trailer, many path-following solutions rely on Jacobian linearization and linear control techniques (Altafini et al., 2001; Divelbiss and Wen, 1997; Hoel and Falcone, 2013). A similar approach for path following is proposed in Rimmer and Cebon (2017) for reversing a general 2trailer with a car-like tractor, which has been successfully demonstrated in practice. A historic survey of path-following techniques for tractor-trailer vehicles can be found in David and Manivannan (2014).

\subsubsection{Path-following control using LQ control: An example}

To conclude this chapter, a path-following example for a kinematic bicycle model is presented. The model of the vehicle is given in (2.11) where the state vector is $x(t)=\left[\begin{array}{lll}x_{1}(t) & y_{1}(t) & \theta_{1}(t)\end{array}\right]^{\top}$, and the control input is $u(t)=\left[\begin{array}{ll}v_{1}(t) & \kappa(t)\end{array}\right]^{\top}$. Here, $v_{1}(t)$ is the longitudinal velocity of the vehicle's rear axle and $\kappa(t)=\tan \alpha(t) / L_{1}$ is its curvature. The wheelbase of the vehicle is $L_{1}$ and $\alpha(t)$ is its steering angle. The kinematic bicycle model perfectly fits into the path-following framework presented in Section 4.3. The progression $s(t)$ is defined as the location of the vehicle's rear axle $\left(x_{1}(t), y_{1}(t)\right)$ onto its projection to its nominal path in $\left(x_{1, r}(\cdot), y_{1, r}(\cdot)\right)$ at time $t$, see Figure 4.7. The orientation of the vehicle evolves as $\dot{\theta}_{1}(t)=v_{1}(t) \kappa(t)$ and by inserting this in (4.34), the complete model of the vehicle in the Frenet-Serret frame (4.35) is

$$
\begin{aligned}
\dot{s} & =v_{1} \frac{\cos \tilde{\theta}_{1}}{1-\kappa_{r}(s) \tilde{z}}, \\
\dot{\tilde{z}} & =v_{1} \sin \tilde{\theta}_{1} \\
\dot{\tilde{\theta}}_{1} & =v_{1}\left(\kappa-\frac{\kappa_{r}(s) \cos \tilde{\theta}_{1}}{1-\kappa_{r}(s) \tilde{z}}\right) .
\end{aligned}
$$

The longitudinal model is represented by (4.41a) and the path-following error model by (4.41b)-(4.41c). For simplicity, we only consider forward motion and assume that $v_{1, r}(t)>0$ and $v_{1}(t)>0$. In this setup, the path-following error is $\tilde{x}=\left[\begin{array}{ll}\tilde{z} & \tilde{\theta}_{1}\end{array}\right]^{\top}$. Denote the controlled curvature deviation as $\tilde{\kappa}=\kappa-\kappa_{r}$ and the path-following error model in (4.41b)-(4.41c) can in a compact form be written as

$$
\dot{\tilde{x}}=v_{1} f_{e}(s(t), \tilde{x}, \tilde{\kappa})
$$

where the origin $(\tilde{x}, \tilde{\kappa})=(0,0)$ is an equilibrium point. Since $v_{1}(t)$ enters bilinearly in (4.42), in analogy with Section 2.3, time-scaling (Sampei and Furuta, 1986) can be applied to eliminate the speed dependence $\left|v_{1}(t)\right|$ from the model. Thus, during the design of the path-following controller it is, without loss of generality, 


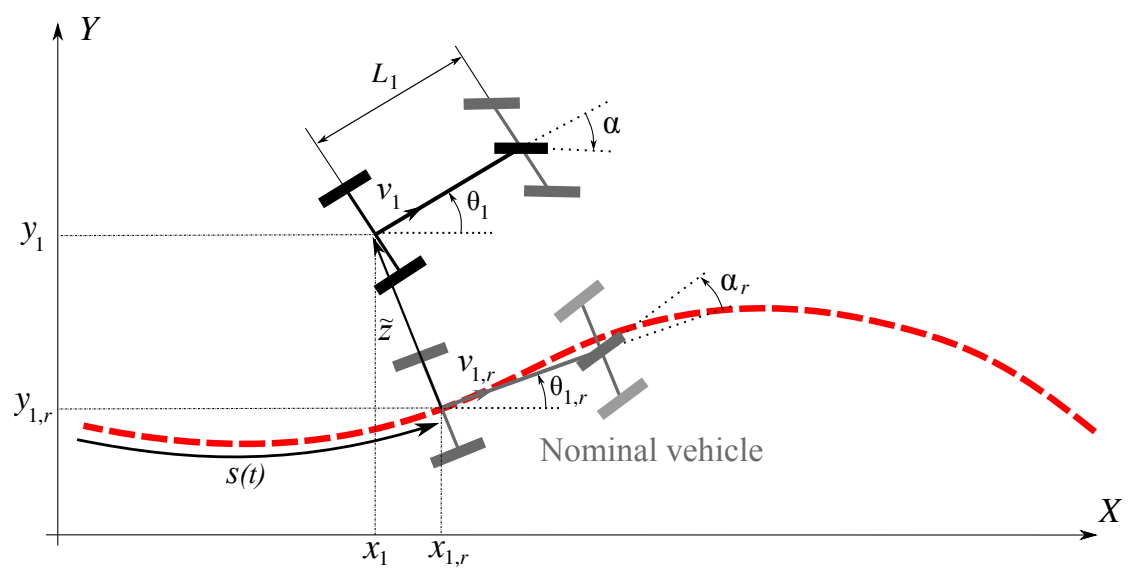

Figure 4.7: The kinematic bicycle model in the Frenet-Serret frame where the red-dashed line represents the nominal path in $\left(x_{1, r}(s), y_{1, r}(s)\right)$. The black vehicle illustrates the controlled system and the gray vehicle is the reference vehicle at $s(t)$.

assumed $^{4}$ that the longitudinal velocity $v_{1}(t)$ of the vehicle only takes on the value $v_{1}(t)=1 \mathrm{~m} / \mathrm{s}$.

The design of the path-following controller is now equivalent to designing a stabilizing feedback controller $\kappa(t)=g(s(t), \tilde{x}(t))$ for the nonlinear system in (4.42) and any method presented in Section 4.2 can now be used with minor adjustments. As an example, we design the path-following controller using robust linear control techniques. Since the origin to (4.42) is an equilibrium point, the Jacobian linearization is

$$
\dot{\tilde{x}}(t)=A(s(t)) \tilde{x}+B \tilde{\kappa},
$$

where

$$
A(s(t))=\left.\frac{\partial f_{e}}{\partial \tilde{x}}\right|_{(0,0)}=\left[\begin{array}{cc}
0 & 1 \\
-\kappa_{r}^{2}(s(t)) & 0
\end{array}\right], \quad B=\left.\frac{\partial f_{e}}{\partial \tilde{\kappa}}\right|_{(0,0)}=\left[\begin{array}{l}
0 \\
1
\end{array}\right] .
$$

The objective is to design a static state-feedback controller with feedforward action $\kappa(t)=\kappa_{r}(s(t))+K \tilde{x}(t)$ such that the closed-loop system

$$
\dot{\tilde{x}}(t)=(A(s(t))+B K) \tilde{x}(t),
$$

is exponentially stable. Assume the nominal path is constructed such that the nominal curvature is bounded as $\left|\kappa_{r}(s(t))\right| \leq \kappa_{\max }$. Then, similar to (4.12), we can describe the linearized path-following error model in (4.43) as a polytopic LDI. The matrix $B$ is a constant and the matrix $A(s(t))$ can be contained in a polytope (4.11) with 2 vertices

$$
A(s(t)) \in \mathbb{P}=\mathbf{C o}\left\{A_{1}, A_{2}\right\},
$$

\footnotetext{
${ }^{4}$ Note that in practice, this assumption is only feasible for low-speed maneuvers since the model is derived based on no-slip conditions and rate-limitations on the steering angle are neglected.
} 
where

$$
A_{1}=\left[\begin{array}{ll}
0 & 1 \\
0 & 0
\end{array}\right], \quad A_{2}=\left[\begin{array}{cc}
0 & 1 \\
-\kappa_{\max }^{2} & 0
\end{array}\right] .
$$

Similarly to (4.14), with a constant decay rate $\epsilon>0$, the LMI feasibility problem to find a stabilizing feedback gain $K=Y S^{-1}$ and a quadratic Lyapunov function $V(\tilde{x})=\tilde{x}^{\top} S^{-1} \tilde{x}$ with $S^{-1} \succ 0$ is

$$
S A_{i}^{\top}+Y^{\top} B^{\top}+A_{i} S+B Y+2 \epsilon S \preceq 0, \quad i=1,2 .
$$

It is desired that the feedback gain $K$ inherits properties of an infinite-horizon LQ controller. A nominal LQ controller is thus designed around a straight reference path $\left(\kappa_{r}(s)=0\right)$ in forward motion using the weight matrices $R=1$ and $Q=\frac{1}{20} \operatorname{diag}([1,1])$. The resulting feedback gain is $K_{\mathrm{LQ}}=-\left[\begin{array}{ll}0.22 & 0.70\end{array}\right]$. This controller is not guaranteed to stabilize the LPV system in (4.43) for all nominal curvatures. To establish stability guarantees, we can minimize the difference between $K$ and $K_{\mathrm{LQ}}$ subject to the LMI in (4.48). Since $S \succ 0$, this can be posed as an SDP problem ${ }^{5}$ (Ljungqvist et al., 2018)

$$
\begin{aligned}
& \underset{Y, S}{\operatorname{minimize}}\left\|Y-K_{\mathrm{LQ}} S\right\| \\
& \text { subject to (4.48) and } S \succ I .
\end{aligned}
$$

The SDP problem in (4.49) is solved using YALMIP (Löfberg, 2004) together with the SDP solver MOSEK (MOSEK, 2019). By selecting $\epsilon=0.2$ and $\kappa_{\max }=0.3$ $\mathrm{m}^{-1}$, the optimal objective function value to $(4.49)$ is zero. This implies that $K=K_{\mathrm{LQ}}$ and one quadratic Lyapunov matrix $P=S^{-1} \succ 0$ is

$$
P=\left[\begin{array}{ll}
0.16 & 0.17 \\
0.17 & 0.63
\end{array}\right]
$$

that guarantees that the closed-loop LTV system $\tilde{x}(t)=(A(s(t))+B K) \tilde{x}(t)$ is exponentially stable as long as the nominal curvature is bounded as $\left|\kappa_{r}(s(t))\right| \leq$ $\kappa_{\max }$. Moreover, with this feedback-law $\tilde{\kappa}(t)=K \tilde{x}(t)$, the origin of the closedloop version of the path-following error system in (4.42) is an exponentially stable equilibrium point when $v_{1}(t)>0$.

To illustrate how the path-following controller handles disturbance rejection, a simulation of the nonlinear closed-loop system is performed. The nominal path is generated using a constant longitudinal velocity profile $v_{1, r}(s)=1$ and a sinusoidal curvature profile $\kappa_{r}(s)=0.2 \sin (0.1 s)$. Figure 4.8 provides simulation results with initial path-following error $\tilde{x}(0)=\left[\begin{array}{lll}\tilde{z}(0) & \tilde{\theta}_{1}(0)\end{array}\right]^{\top}=\left[\begin{array}{lll}1 & \mathrm{~m} & 0.2 \mathrm{rad}\end{array}\right]^{\top}$. As can be seen, the initial lateral and angular path-following errors are eliminated after roughly $15 \mathrm{~s}$. The value of the quadratic Lyapunov function $V(\tilde{x}(t))=\tilde{x}^{\top}(t) P \tilde{x}(t)$ for the simulation is provided in Figure 4.9. The result shows that the closed-loop system is exponentially stable. Note that a similar control design can be performed for backward motion by simply changing the sign of the matrices $A(s(t))$ and $B$ in (4.43). However, it is not possible to design the linear state-feedback controllers such that a common quadratic Lyapunov function exists in forward and backward motion. This will be shown in Paper C.

\footnotetext{
${ }^{5}$ If $\left\|K-K_{\mathrm{LQ}}\right\|=\left\|Y S^{-1}-K_{\mathrm{LQ}}\right\|=\left\|\left(Y-K_{\mathrm{LQ}} S\right) S^{-1}\right\|=0$, then $K=K_{\mathrm{LQ}}$ since $S \succ 0$.
} 


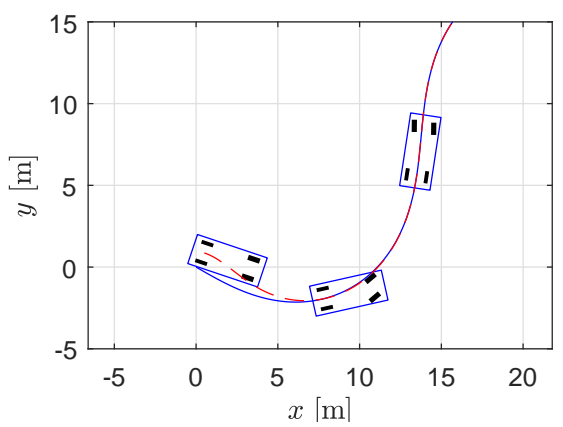

(a) The nominal path (blue line) and the motion of the controlled vehicle (reddashed line).

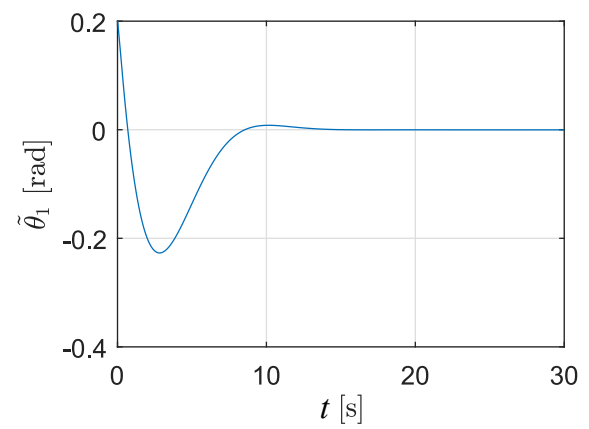

(c) The orientation error $\tilde{\theta}_{1}(t)$.

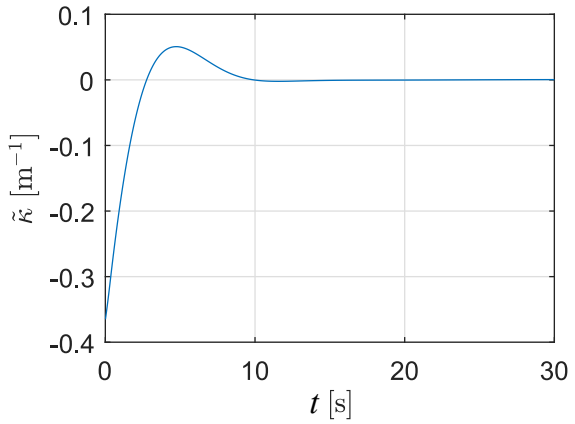

(b) Feedback-control signal $\tilde{\kappa}(t)=K \tilde{x}(t)$.

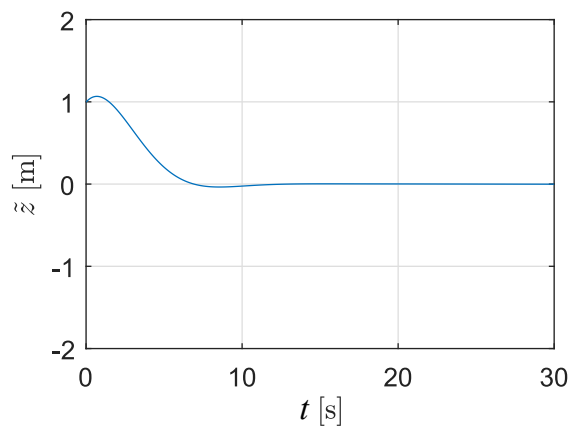

(d) The lateral error $\tilde{z}(t)$.

Figure 4.8: Simulation results for the closed-loop system using a pathfollowing controller designed using $L Q$ control.

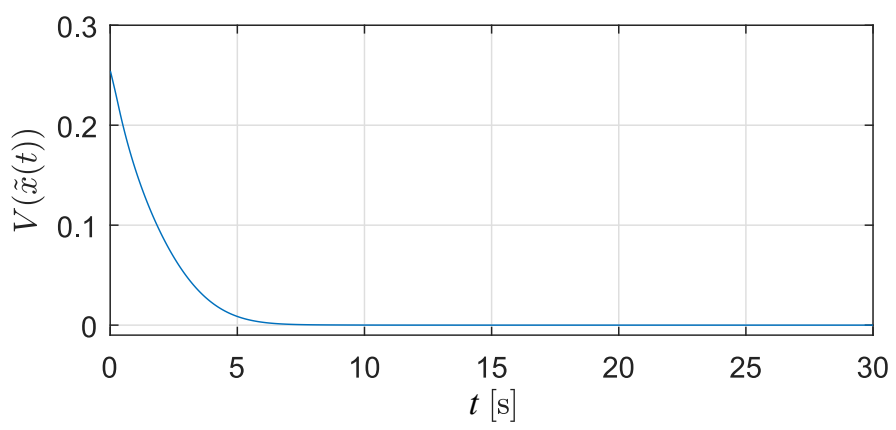

Figure 4.9: The quadratic Lyapunov function $V(\tilde{x}(t))=\tilde{x}^{\top}(t) P \tilde{x}(t)$ during the simulation. 



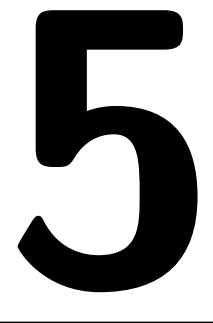

\section{Concluding remarks}

In this chapter, a summary of the contributions of this thesis is given in more technical terms and some directions for future work are discussed. The first part of this thesis gave an introduction to nonholonomic systems and some common motion planning and feedback control techniques for such systems. These techniques have been combined and modified for several practical problems and the results are presented in the papers that constitute the second part of this thesis.

\subsection{Summary of contributions}

The contributions of this thesis are within the area of motion planning and feedback control for different kinds of tractor-trailer combinations during low-speed maneuvers in unstructured environments. The proposed motion planning techniques include probabilistic and deterministic sampling-based approaches. Furthermore, numerical optimal control techniques are used offline to precompute libraries of optimized maneuvers as well as during online planning as a warm-started optimization step. To follow the computed motion plans, different path-following control approaches are proposed that use a path-following error model of the vehicle. Several of the proposed approaches have been successfully demonstrated on a small-scale or on a full-scale general 2-trailer with a car-like tractor. Furthermore, the first complete motion planning and control framework for this type of system is presented which is also accompanied with the first successfully results from real-world experiments. In this framework, a novel nonlinear observer for state estimation is evaluated which only utilizes information from sensors mounted on the tractor, making the framework independent of any sensor mounted on the trailer.

In Paper A, a probabilistic motion planner for a general 2-trailer with a car-like tractor is proposed. The cascade-like path-following controller presented in Evestedt et al. (2016a); Ljungqvist (2015) is used to enable efficient closed-loop simu- 
lations of the vehicle within a CL-RRT framework. The framework is evaluated in a series of Monte-Carlo runs and is shown to have a high success rate in finding motion plans in complex and constrained environments. Experiments on a small-scale test platform is also presented.

In Paper B, a path-following controller for a reversing general 2-trailer with a car-like tractor is proposed for the case when the nominal path contains full state and control-input information. A path-following error model of the vehicle is derived and a stabilizing LQ controller with feedforward action is proposed. Given that the nominal path satisfies certain properties, exponential stability of the closed-loop system is established by combining global optimization, theory from LDIs, and LMI techniques. The theoretical results are verified through simulations of the closed-loop system around a figure-eight shaped reference path.

In Paper C, a systematic framework is proposed for analyzing stability of the closed-loop system consisting of a controlled vehicle and a feedback controller, executing a motion plan computed by a lattice-based planner. When this motion planner is considered, it is shown that the closed-loop system can be modeled as a time-switched nonlinear hybrid system. Based on this, a method is proposed for analyzing the behavior of the tracking error, how to design the low-level controller and how to potentially impose restrictions on the motion planner to guarantee that the tracking error is bounded and decays towards zero. The synthesis and analysis tools are based on LMI techniques. The proposed method is applied on a general 2-trailer with a car-like tractor and the results are illustrated in simulations.

In Paper D, a path-planning and path-following control framework for a general 2-trailer with a car-like tractor is presented. A lattice-based path planner is developed that uses a library of optimized maneuvers together with graph search to compute a path to the goal. To follow the planned path, the LQ controller in Paper B is extended and used in practice. To estimate the vehicle states needed for control, a nonlinear observer is developed which only utilizes information from a rear-view LIDAR sensor mounted on the tractor, making the system independent of any sensor mounted on the semitrailer or the dolly. The proposed framework is implemented on a full-scale test vehicle and results from simulations and field experiments are presented.

In Paper E, an estimation-aware MPC controller for a general 2-trailer with a car-like tractor is proposed. The approach is driven and inspired by the work in Paper D where an advanced sensor with a limited field of view is placed in the rear of the tractor to solve the joint-angle estimation problem. To model and explicitly consider the sensor's sensing limitations in the controller, a model predictive path-following control approach is proposed. Two approaches with different computation complexity and performance are presented. In the first approach, the constraint on the joint angles is modeled as a union of convex polytopes, making it necessary to incorporate binary decision variables in the MPC controller. The second approach, which is a special case of the former, avoids binary variables at the expense of a more restrictive approximation of the joint-angle constraints. The performance of the proposed path-following control approach is evaluated in simulations as well as in field experiments on a full-scale test vehicle.

In Paper F, a two-step optimization-based trajectory planner is proposed for 
MSNT vehicles operating at low-speeds. The proposed trajectory planner uses a lattice-based trajectory planner in a first step to compute a resolution optimal solution to the trajectory planning problem. The output from the lattice-based planner is then used to warm-start a second numerical optimal control step, which enables the framework to compute locally optimal trajectories that start at the vehicle's initial state and reaches the goal state exactly. The performance of the proposed optimization-based trajectory planner is evaluated in a set of parking scenarios for a MS3T vehicle with a car-like tractor where the last trailer is steerable.

Finally, in Paper G, a model predictive path-following controller is proposed for automatic low-speed control of MSNT vehicles. The proposed MPC controller uses a linear path-following error model of the vehicle for predictions and is designed to satisfy various constraints on the vehicle states as well as saturations and rate limitations on the tractor's curvature and the trailer steering angles. The performance of the proposed model predictive path-following controller is evaluated in a set of simulations for a MS2T vehicle with a car-like tractor where the last trailer is steerable.

\subsection{Future work}

In this section, some ideas for future work and possible extensions of the contributions that are presented in this thesis are summarized.

\section{Motion planning and feedback control}

The CL-RRT algorithm developed for a general 2-trailer with a car-like tractor in Paper A is possible to generalize to the general N-trailer and the MSNT cases. It would also be interesting to benchmark its performance, as well as the lattice-based approaches, with previously presented motion planning strategies for standard Ntrailer vehicles, e.g., Laumond et al. (1994); Sekhavat et al. (1998).

To increase the computational efficiency of the two-step optimization-based motion planner in Paper F, it is possible to run the optimization step in receding horizon as proposed in Bergman et al. (2020a). Using this technique, it would be interesting to let the optimization step perform local adjustments of the motion plan to become reactive against, e.g., inaccurate predictions of moving obstacles or if previously unseen obstacles are detected by the perception layer (Batkovic et al., 2019, 2020). In such a reactive setup, it will be important to understand and analyze which layer, i.e., sampling-based planner or optimization step, that is responsible for modifying the motion plan to obtain guarantees in terms of feasibility and convergence to the goal. It would also be interesting to investigate if the optimization-based motion planning concept can be applied on other systems, such as drones (Andersson et al., 2018b) and ships (Bergman et al., 2020b).

The estimation-aware control concept presented in Paper E would be interesting to extend to other systems as well as other scenarios where onboard or external sensors with similar sensing limitations are used to enhance the performance of the vehicle's estimation and perception layers. As an example, one practical scenario is reversing a tractor-trailer vehicle to a loading bay where, e.g., external sensors 
placed on the loading bay can be used to improve the performance of the estimation and control modules. These sensing limitations can also be incorporated in the motion planner either as constraints or using techniques from informative path planning and sensor management (Boström-Rost, 2019) to, e.g., optimize the system's future perception of the surrounding environment.

Onroad motion planning for tractor-trailer vehicles is also an interesting and natural research direction to pursuit. In forward motion, the joint-angle kinematics are stable and do not necessarily need to be stabilized. However, during evasive maneuvers, a dynamic vehicle model needs to be used in order to capture dynamic effects and to compute motion plans that are guaranteed to be feasible to follow. As long as the system can be simulated, it would be possible to develop RRT frameworks or lattice-based frameworks using input-sampling for this task. Another possibility is to leave more responsibility to the feedback controller to perform both local planning and control. Other interesting aspects related to onroad motion planning for tractor-trailer vehicles are interaction-aware trajectory planning (Evestedt, 2016; Ward et al., 2017), fall-back solutions using safe-stop trajectory planning (Svensson et al., 2018), as well as methods to incorporate human-like driving behaviors (Rosbach et al., 2019) and traffic rules (Tumova et al., 2013).

Robust and safe trajectory planning (Althoff and Dolan, 2014; Majumdar and Tedrake, 2017; Singh et al., 2018), lateral control of tractor-trailer vehicles at high speeds and during at the limit-handling (Anistratov et al., 2018; Berntorp et al., 2014; Fors et al., 2019; Pereira et al., 2018; Svensson et al., 2019) are also interesting future research directions. Example of disturbances that the system need to be robust against include parameter uncertainties, strong winds and slippery road surface conditions. In a lattice-based motion planning framework, it would be interesting to compute several libraries of maneuvers that are robust against different values of the disturbances. During online planning, the current estimate of the disturbances can then be used to select the most appropriate library. Compared to computing only one library for any type of disturbance as proposed in Majumdar and Tedrake (2017), the uncertainties of slowly-varying disturbances can be reduced. As a result, the motion planner will become less conservative without increasing the risk of inducing instability or collision with surrounding obstacles.

\section{Learning and system identification}

The incorporation of learning in a motion planning and control architecture is also an interesting direction of future research. If similar motion plans are used multiple times, it would be possible to evaluate and adapt the controller and/or the motion plans in order to enhance the performance of the closed-loop system (Rosolia and Borrelli, 2017). These ideas have strong similarities with iterative learning control (ILC) (Bristow et al., 2006; Gunnarsson and Norrlöf, 2001) and learning MPC (Rosolia and Borrelli, 2017; Rosolia et al., 2017) tailored for repetitive tasks.

It would also be interesting to perform online estimation of important vehicle parameters, such as road friction coefficients and side-slip angles (Morrison and Cebon, 2016; Svensson et al., 2019), moment of inertia (Cheng and Cebon, 
2011) and vehicle lengths (Arnström, 2018). Reinforcement learning approaches are also interesting directions for future work where, e.g., cost functions, motion primitives and heuristics used in a motion planner could be learned from an expert driver (Rosbach et al., 2019). 

Appendix 



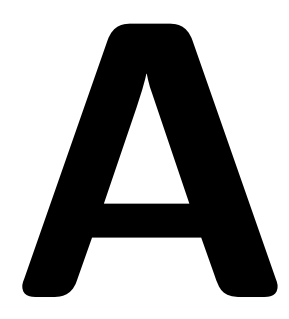

\section{Derivation of the tracking-error system}

In this section, the derivation of the tracking-error system in (4.25) is presented. The kinematic bicycle model in a global coordinate frame is given by

$$
\dot{x}(t)=v_{1}(t)\left[\begin{array}{c}
\cos \theta_{1}(t) \\
\sin \theta_{1}(t) \\
\kappa(t)
\end{array}\right]
$$

where $x(t)=\left[\begin{array}{lll}x_{1}(t) & y_{1}(t) & \theta_{1}(t)\end{array}\right]^{\top}$ is the state vector and $u(t)=\left[\begin{array}{ll}v_{1}(t) & \kappa(t)\end{array}\right]^{\top}$ is the control-input vector. Represent the model in (A.1) as $\dot{x}(t)=f(x(t), u(t))$. The nominal trajectory is feasible and thus satisfies $\dot{x}_{r}(t)=f\left(x_{r}(t), u_{r}(t)\right)$. Denote the tracking error as $\bar{x}(t)=x(t)-x_{r}(t)$. By taking the time derivative of the proposed state transformation

$$
\left[\begin{array}{l}
x_{1, e}(t) \\
y_{1, e}(t) \\
\theta_{1, e}(t)
\end{array}\right]=T(t, \bar{x}(t)) \bar{x}(t)=\left[\begin{array}{ccc}
\cos \theta_{1}(t) & \sin \theta_{1}(t) & 0 \\
-\sin \theta_{1}(t) & \cos \theta_{1}(t) & 0 \\
0 & 0 & 1
\end{array}\right]\left[\begin{array}{l}
\bar{x}_{1}(t) \\
\bar{y}_{1}(t) \\
\bar{\theta}_{1}(t)
\end{array}\right]
$$

and using the relations $\dot{x}_{1} \sin \theta_{1}=\dot{y}_{1} \cos \theta_{1}$ and $v_{1}=\dot{x}_{1} \cos \theta_{1}+\dot{y}_{1} \sin \theta_{1}$, the trackingerror system in (4.25) is

$$
\begin{aligned}
\dot{x}_{1, e} & =\left(\dot{x}_{1}-\dot{x}_{1, r}\right) \cos \theta_{1}+\left(\dot{y}_{1}-\dot{y}_{1, r}\right) \sin \theta_{1}-\left(x_{1}-x_{1, r}\right) \dot{\theta}_{1} \sin \theta_{1}+\left(y_{1}-y_{1, r}\right) \dot{\theta}_{1} \cos \theta_{1} \\
& =v_{1}+y_{1, e} v_{1} \kappa-\dot{x}_{1, r} \cos \theta_{1}-\dot{y}_{1, r} \sin \theta_{1} \\
& =v_{1}+y_{1, e} v_{1} \kappa-\dot{x}_{1, r} \cos \left(\theta_{1, r}+\theta_{1, e}\right)-\dot{y}_{1, r} \sin \left(\theta_{1, r}+\theta_{1, e}\right) \\
& =v_{1}+y_{1, e} v_{1} \kappa-\dot{x}_{1, r}\left(\cos \theta_{1, r} \cos \theta_{1, e}-\sin \theta_{1, r} \sin \theta_{1, e}\right)-\dot{y}_{1, r}\left(\sin \theta_{1, r} \cos \theta_{1, e}+\cos \theta_{1, r} \sin \theta_{1, e}\right) \\
& =v_{1}+y_{1, e} v_{1} \kappa-\left(\dot{x}_{1, r} \cos \theta_{1, r}+\dot{y}_{1, r} \sin \theta_{1, r}\right) \cos \theta_{1, e}+\left(\dot{x}_{1, r} \sin \theta_{1, r}-\dot{y}_{1, r} \cos \theta_{1, r}\right) \cos \theta_{1, e} \\
& =v_{1}+y_{1, e} v_{1} \kappa-v_{1, r} \cos \theta_{1, e},
\end{aligned}
$$




$$
\begin{aligned}
\dot{y}_{1, e} & =-\left(\dot{x}_{1}-\dot{x}_{1, r}\right) \sin \theta_{1}+\left(\dot{y}_{1}-\dot{y}_{1, r}\right) \cos \theta_{1}-\left(x_{1}-x_{1, r}\right) \dot{\theta}_{1} \cos \theta_{1}-\left(y_{1}-y_{1, r}\right) \dot{\theta}_{1} \sin \theta_{1} \\
& =-x_{1, e} v_{1} \kappa+\dot{x}_{1, r} \sin \left(\theta_{1, r}+\theta_{1, e}\right)-\dot{y}_{1, r} \cos \left(\theta_{1, r}+\theta_{1, e}\right) \\
& =-x_{1, e} v_{1} \kappa+\dot{x}_{1, r}\left(\sin \theta_{1, r} \cos \theta_{1, e}+\sin \theta_{1, e} \cos \theta_{1, r}\right)-\dot{y}_{1, r}\left(\cos \theta_{1, r} \cos \theta_{1, e}-\sin \theta_{1, r} \sin \theta_{1, e}\right) \\
& =-x_{1, e} v_{1} \kappa+\left(\dot{x}_{1, r} \sin \theta_{1, r}-\dot{y}_{1, r} \cos \theta_{1, r}\right) \cos \theta_{1, e}+\left(\dot{x}_{1, r} \cos \theta_{1, r}+\dot{y}_{1, r} \sin \theta_{1, r}\right) \sin \theta_{1, e} \\
& =-x_{1, e} v_{1} \kappa+v_{1, r} \sin \theta_{1, e},
\end{aligned}
$$

and

$$
\dot{\theta}_{1, e}=\dot{\bar{\theta}}_{1}=\dot{\theta}_{1}-\dot{\theta}_{1, r}=v_{1} \kappa-v_{1, r} \kappa_{r} .
$$

Together, the model in (A.3)-(A.5) represents the tracking-error system (4.25) in the local coordinate frame. 


\section{Bibliography}

C. Altafini. Some properties of the general n-trailer. International Journal of Control, 74(4):409-424, 2001.

C. Altafini. Path following with reduced off-tracking for multibody wheeled vehicles. IEEE Transactions on Control Systems Technology, 11(4):598-605, 2003.

C. Altafini, A. Speranzon, and B. Wahlberg. A feedback control scheme for reversing a truck and trailer vehicle. IEEE Transactions on Robotics and Automation, 17(6):915-922, 2001.

C. Altafini, A. Speranzon, and K-H. Johansson. Hybrid control of a truck and trailer vehicle. In Hybrid Systems: Computation and Control, pages 21-34. Springer, 2002.

M. Althoff and J. M Dolan. Online verification of automated road vehicles using reachability analysis. IEEE Transactions on Robotics, 30(4):903-918, 2014.

B. Anderson and J. B. Moore. Optimal control: linear quadratic methods. Courier Corporation, 2007.

J. A E Andersson, J. Gillis, G. Horn, J. B Rawlings, and M. Diehl. CasADi - A software framework for nonlinear optimization and optimal control. Mathematical Programming Computation, 2018a.

O. Andersson, O. Ljungqvist, M. Tiger, D. Axehill, and F. Heintz. Recedinghorizon lattice-based motion planning with dynamic obstacle avoidance. In Proceedings of the 57th IEEE Conference on Decision and Control, pages 44674474, 2018b.

P. Anistratov, B. Olofsson, and L. Nielsen. Segmentation and merging of autonomous at-the-limit maneuvers for ground vehicles. In Proceedings of the International Symposium on Advanced Vehicle Control, 2018.

D. Arnström. State estimation for truck and trailer systems using deep learning. Master's thesis, Linköping University, 2018. 
A. Astolfi, P. Bolzern, and A. Locatelli. Path-tracking of a tractor-trailer vehicle along rectilinear and circular paths: A Lyapunov-based approach. IEEE Transactions on Robotics and Automation, 20(1):154-160, 2004.

D. Axehill. Applications of Integer Quadratic Programming in Control and Communication. Licentiate's thesis, Linköping University, 2005.

I. Batkovic, M. Zanon, M. Ali, and P. Falcone. Real-time constrained trajectory planning and vehicle control for proactive autonomous driving with road users. In Proceedings of the 18th European Control Conference, pages 256-262, 2019.

I. Batkovic, M. Ali, P. Falcone, and M. Zanon. Safe trajectory tracking in uncertain environments. arXiv preprint arXiv:2001.11602, 2020.

K. Bergman, O. Ljungqvist, and D. Axehill. Improved path planning by tightly combining lattice-based path planning and optimal control. Accepted for publication in IEEE Transactions on Intelligent Vehicles. Preprint: https: //arxiv.org/abs/1903.07900, 2019a.

K. Bergman, O. Ljungqvist, and D. Axehill. Improved optimization of motion primitives for motion planning in state lattices. In Proceedings of the 2019 IEEE Intelligent Vehicles Symposium, 2019b.

K. Bergman, O. Ljungqvist, T. Glad, and D. Axehill. An optimization-based receding horizon trajectory planning algorithm. Accepted for publication in Proceedings of the 21th IFAC World Congress. Preprint: https://arxiv.org/ abs/1912.05259, 2020a.

K. Bergman, O. Ljungqvist, J. Linder, and D. Axehill. An optimization-based motion planner for autonomous maneuvering of marine vessels in complex environments. Submitted to the 59th IEEE Conference on Decision and Control, 2020 b.

K. Berntorp, B. Olofsson, K. Lundahl, and L. Nielsen. Models and methodology for optimal trajectory generation in safety-critical road-vehicle manoeuvres. Vehicle System Dynamics, 52(10):1304-1332, 2014.

R. L Bishop. There is more than one way to frame a curve. The American Mathematical Monthly, 82(3):246-251, 1975.

P. Bolzern, R. M. DeSantis, A. Locatelli, and D. Masciocchi. Path-tracking for articulated vehicles with off-axle hitching. IEEE Transactions on Control Systems Technology, 6(4):515-523, 1998.

P. Boström-Rost. On Informative Path Planning for Tracking and Surveillance. Licentiate's thesis, Linköping University, 2019.

S. P. Boyd, L. Ghaoui, E. Feron, and V. Balakrishnan. Linear matrix inequalities in system and control theory, volume 15. SIAM, 1994. 
D. A Bristow, M. Tharayil, and A. G Alleyne. A survey of iterative learning control. IEEE Control Systems Magazine, 26(3):96-114, 2006.

M. Buehler, K. Iagnemma, and S. Singh. The 2005 DARPA grand challenge: the great robot race, volume 36. Springer, 2007.

M. Buehler, K. Iagnemma, and S. Singh. The DARPA urban challenge: autonomous vehicles in city traffic, volume 56. springer, 2009.

L. D. Burns. Sustainable mobility: a vision of our transport future. Nature, 497 (7448):181, 2013.

S. Di Cairano, U. V. Kalabić, and K. Berntorp. Vehicle tracking control on piecewise-clothoidal trajectories by MPC with guaranteed error bounds. In Proceedings of the 55th IEEE Conference on Decision and Control, pages 709-714, 2016.

C. Cheng and D. Cebon. Parameter and state estimation for articulated heavy vehicles. Vehicle System Dynamics, 49(1-2):399-418, 2011.

M. Cirillo, T. Uras, and S. Koenig. A lattice-based approach to multi-robot motion planning for non-holonomic vehicles. In Proceedings of the 2014 IEEE/RSJ International Conference on Intelligent Robots and Systems, pages 232-239, 2014.

C. R Coulter. Implementation of the pure pursuit path tracking algorithm. Technical report, Carnegie-Mellon UNIV Pittsburgh PA Robotics INST, 1992.

J. David and P. V. Manivannan. Control of truck-trailer mobile robots: a survey. Intelligent Service Robotics, 7(4):245-258, 2014.

N. Deo and M. M Trivedi. Multi-modal trajectory prediction of surrounding vehicles with maneuver based LSTMs. In Proceedings of the 2018 IEEE Intelligent Vehicles Symposium, pages 1179-1184, 2018.

N. Deo, A. Rangesh, and M. M Trivedi. How would surround vehicles move? A unified framework for maneuver classification and motion prediction. IEEE Transactions on Intelligent Vehicles, 3(2):129-140, 2018.

A. W. Divelbiss and J. T. Wen. Trajectory tracking control of a car-trailer system. IEEE Transactions on Control Systems Technology, 5(3):269-278, 1997.

D. Dolgov, S. Thrun, M. Montemerlo, and J. Diebel. Path planning for autonomous vehicles in unknown semi-structured environments. The International Journal of Robotics Research, 29(5):485-501, 2010.

J. Doyle. Guaranteed margins for LQG regulators. IEEE Transactions on Automatic Control, 23(4):756-757, 1978.

L. E Dubins. On curves of minimal length with a constraint on average curvature, and with prescribed initial and terminal positions and tangents. American Journal of Mathematics, 79(3):497-516, 1957. 
A. Elfes. Using occupancy grids for mobile robot perception and navigation. Computer, 22(6):46-57, 1989.

Ernst and Young. Who's in the driving seat? How the rise of autonomous vehicles will transform the relationship between man and car. Technical report, 2015.

European Commission. Roadmap to a single european transport area - Towards a competitive and resource efficient transport system. Technical report, 2011.

European Road Transport Research Advisory Council. Multi-annual implementation plan for Horizon 2020. Technical report, 2013.

N. Evestedt. Sampling Based Motion Planning for Heavy Duty Autonomous Vehicles. Licentiate's thesis, Linköping University, 2016.

N. Evestedt, D. Axehill, M. Trincavelli, and F. Gustafsson. Sampling recovery for closed loop rapidly expanding random tree using brake profile regeneration. In 2015 IEEE Intelligent Vehicles Symposium, pages 101-106, 2015.

N. Evestedt, O. Ljungqvist, and D. Axehill. Path tracking and stabilization for a reversing general 2-trailer configuration using a cascaded control approach. In Proceedings of the 2016 IEEE Intelligent Vehicles Symposium, pages 1156-1161, 2016a.

N. Evestedt, O. Ljungqvist, and D. Axehill. Motion planning for a reversing general 2-trailer configuration using Closed-Loop RRT. In Proceedings of the 2016 IEEE/RSJ International Conference on Intelligent Robots and Systems, pages 3690-3697, 2016b.

P. Falcone, F. Borrelli, J. Asgari, E. H Tseng, and D. Hrovat. Predictive active steering control for autonomous vehicle systems. IEEE Transactions on Control Systems Technology, 15(3):566-580, 2007.

H. J Ferreau, C. Kirches, A. Potschka, H. G Bock, and M. Diehl. qpOASES: A parametric active-set algorithm for quadratic programming. Mathematical Programming Computation, 6(4):327-363, 2014.

M. Fliess, J. Lévine, P. Martin, and P. Rouchon. Design of trajectory stabilizing feedback for driftless flat systems. Proceedings of the Third European Control Conference, pages 1882-1887, 1995.

V. Fors, B. Olofsson, and L. Nielsen. Formulation and interpretation of optimal braking and steering patterns towards autonomous safety-critical manoeuvres. Vehicle system dynamics, 57(8):1206-1223, 2019.

T. Fraichard and A. Scheuer. From Reeds and Shepp's to continuous-curvature paths. IEEE Transactions on Robotics, 20(6):1025-1035, 2004.

E. Frazzoli, M. A. Dahleh, and E. Feron. Maneuver-based motion planning for nonlinear systems with symmetries. IEEE Transactions on Robotics, 21(6): 1077-1091, 2005. 
D. J Gammell. Informed anytime search for continuous planning problems. PhD thesis, University of Toronto, 2017.

C. F Garcia, D. M Prett, and M. Morari. Model predictive control: theory and practice - a survey. Automatica, 25(3):335-348, 1989.

P. E Gill, W. Murray, and M. A Saunders. SNOPT: An SQP algorithm for largescale constrained optimization. SIAM review, 47(1):99-131, 2005.

K. Granström, M. Fatemi, and L. Svensson. Poisson multi-bernoulli mixture conjugate prior for multiple extended target filtering. IEEE Transactions on Aerospace and Electronic Systems, 2019.

S. Gros, M. Zanon, R. Quirynen, A. Bemporad, and M. Diehl. From linear to nonlinear MPC: bridging the gap via the real-time iteration. International Journal of Control, 93(1):62-80, 2020.

S. Gunnarsson and M. Norrlöf. On the design of ILC algorithms using optimization. Automatica, 37(12):2011-2016, 2001.

P. E Hart, N. J Nilsson, and B. Raphael. A formal basis for the heuristic determination of minimum cost paths. IEEE Transactions on Systems Science and Cybernetics, 4(2):100-107, 1968.

A. Helmersson. Methods for robust gain scheduling. PhD thesis, Linköping University, 1995.

C-J Hoel and P. Falcone. Low speed maneuvering assistance for long vehicle combinations. In Proceedings of the 2013 IEEE Intelligent Vehicles Symposium, pages 598-604, 2013.

A. Hornung, K. M Wurm, M. Bennewitz, C. Stachniss, and W. Burgard. OctoMap: An efficient probabilistic 3D mapping framework based on octrees. Autonomous Robots, 34(3):189-206, 2013.

A. Houenou, P. Bonnifait, V. Cherfaoui, and M. Yao. Vehicle trajectory prediction based on motion model and maneuver recognition. In Proceedings of the 2013 IEEE/RSJ International Conference on Intelligent Robots and Systems, pages 4363-4369, 2013.

B. Houska, H. J Ferreau, and M. Diehl. An auto-generated real-time iteration algorithm for nonlinear MPC in the microsecond range. Automatica, 47(10): 2279 - 2285, 2011a.

B. Houska, H. J. Ferreau, and M. Diehl. ACADO Toolkit - An open source framework for automatic control and dynamic optimization. Optimal Control Applications and Methods, 32(3):298-312, $2011 \mathrm{~b}$.

M. Kalisiak and M. van de Panne. RRT-blossom: RRT with a local flood-fill behavior. In Proceedings of the 2006 IEEE International Conference on Robotics and Automation, pages 1237-1242, 2006. 
R. E Kalman et al. Contributions to the theory of optimal control. Boletin de la Sociedad Matematica Mexicana, 5(2):102-119, 1960.

I. Kaminer, O. Yakimenko, A. Pascoal, and R. Ghabcheloo. Path generation, path following and coordinated control for timecritical missions of multiple UAVs. In Proceedings of the 2006 American Control Conference, pages 4906-4913, 2006.

Y. Kanayama, Y. Kimura, F. Miyazaki, and T. Noguchi. A stable tracking control method for an autonomous mobile robot. In Proceedings of the IEEE International Conference on Robotics and Automation, pages 384-389 vol.1, 1990.

S. Karaman and E. Frazzoli. Incremental sampling-based algorithms for optimal motion planning. Robotics Science and Systems VI, 104:2, 2010.

S. Karaman and E. Frazzoli. Sampling-based algorithms for optimal motion planning. The International Journal of Robotics Research, 30(7):846-894, 2011.

M. S. Kati, H. Köroğlu, and J. Fredriksson. Robust control of an A-double with active dolly based on static output feedback and dynamic feed-forward. In International Forum for Road Transport Technology, 15th International Heavy Vehicle Transport Technology Symposium. Roturua, 2016.

M. S. Kati, J. Fredriksson, B. Jacobson, and L. Laine. Gain-scheduled $\mathrm{H}_{\infty}$ controller synthesis for actively steered longer and heavier commercial vehicles. Proceedings of the Institution of Mechanical Engineers, Part D: Journal of Automobile Engineering, 2019a.

M. S. Kati, H. Köroğlu, and J. Fredriksson. Robust lateral control of longcombination vehicles under moments of inertia and tyre cornering stiffness uncertainties. Vehicle System Dynamics, 57(12):1847-1873, 2019 b.

H. K. Khalil and J. W. Grizzle. Nonlinear systems, volume 3. Prentice hall Upper Saddle River, NJ, 2002.

B. Kim, C. M Kang, J. Kim, S. H Lee, C. C Chung, and J. W Choi. Probabilistic vehicle trajectory prediction over occupancy grid map via recurrent neural network. In Proceedings of the 2017 IEEE 20th International Conference on Intelligent Transportation Systems, pages 399-404, 2017.

R. A Knepper and A. Kelly. High performance state lattice planning using heuristic look-up tables. In Proceedings of the 2006 IEEE/RSJ International Conference on Intelligent Robots and Systems, pages 3375-3380, 2006.

T. Kunz and M. Stilman. Kinodynamic RRTs with fixed time step and best-input extension are not probabilistically complete. In Algorithmic Foundations of Robotics XI, pages 233-244. Springer, 2015.

Y. Kuwata, S. Karaman, J. Teo, E. Frazzoli, J. P. How, and G. Fiore. Realtime motion planning with applications to autonomous urban driving. IEEE Transactions on Control Systems Technology, 17(5):1105-1118, 2009. 
J. SW Lamb and J. AG Roberts. Time-reversal symmetry in dynamical systems: a survey. Physica-Section D, 112(1):1-39, 1998.

J. P. Laumond, P. E. Jacobs, M. Taix, and R. M. Murray. A motion planner for nonholonomic mobile robots. IEEE Transactions on Robotics and Automation, 10(5):577-593, 1994.

S. M. LaValle. Rapidly-exploring random trees a new tool for path planning. Technical report, 1998.

S. M. LaValle. Planning algorithms. Cambridge University Press, 2006.

S. M. LaValle and J. J. Kuffner. Randomized kinodynamic planning. The International Journal of Robotics Research, 20(5):378-400, 2001.

M. Likhachev, G. J. Gordon, and S. Thrun. ARA*: Anytime A* with provable bounds on sub-optimality. In Advances in Neural Information Processing Systems, pages 767-774, 2004.

P. F. Lima. Optimization-Based Motion Planning and Model Predictive Control for Autonomous Driving: With Experimental Evaluation on a Heavy-Duty Construction Truck. PhD thesis, KTH Royal Institute of Technology, Stockholm, 2018.

P. F. Lima, M. Nilsson, M. Trincavelli, J. Mårtensson, and B. Wahlberg. Spatial Model Predictive Control for Smooth and Accurate Steering of an Autonomous Truck. IEEE Transactions on Intelligent Vehicles, 2(4):238-250, 2017a.

P. F. Lima, R. Oliveira, J. Mårtensson, and B. Wahlberg. Minimizing long vehicles overhang exceeding the drivable surface via convex path optimization. In Proceedings of the 20th IEEE International Conference on Intelligent Transportation Systems, 2017b.

G. Ling, K. Lindsten, O. Ljungqvist, J. Löfberg, C. Norén, and C. A. Larsson. Fuelefficient model predictive control for heavy duty vehicle platooning using neural networks. In Proceedings of the 2018 Annual American Control Conference, pages 3994-4001, 2018.

O. Ljungqvist. Motion planning and stabilization for a reversing truck and trailer system. Master's thesis, Linköping University, 2015.

O. Ljungqvist. On motion planning and control for truck and trailer systems. Licentiate's thesis, Linköping University, 2019.

O. Ljungqvist and D. Axehill. A predictive path-following controller for multisteered articulated vehicles. Accepted for publication in Proceedings of the 21th IFAC World Congress. Preprint: https://arxiv.org/abs/1912.06259, 2020.

O. Ljungqvist, D. Axehill, and A. Helmersson. Path following control for a reversing general 2-trailer system. In Proceedings of the 55th IEEE Conference on Decision and Control, pages 2455-2461, 2016. 
O. Ljungqvist, N. Evestedt, M. Cirillo, D. Axehill, and O. Holmer. Lattice-based motion planning for a general 2-trailer system. In Proceedings of the 2017 IEEE Intelligent Vehicles Symposium, pages 2455-2461, 2017.

O. Ljungqvist, D. Axehill, and J. Löfberg. On stability for state-lattice trajectory tracking control. In Proceedings of the 2018 American Control Conference, 2018.

O. Ljungqvist, N. Evestedt, D. Axehill, M. Cirillo, and H. Pettersson. A path planning and path-following control framework for a general 2-trailer with a car-like tractor. Journal of Field Robotics, 36(8):1345-1377, 2019.

O. Ljungqvist, D. Axehill, and H. Pettersson. On sensing-aware model predictive path-following control for a reversing general 2-trailer with a car-like tractor. Accepted for publication in Proceedings of the 2020 IEEE International Conference on Robotics and Automation. Preprint: https://arxiv.org/abs/2002.06874, 2020a.

O. Ljungqvist, D. Axehill, H. Pettersson, and J. Löfberg. Estimation-aware model predictive path-following control for a general 2-trailer with a car-like tractor. Submitted to IEEE Transactions on Robotics. Preprint: https://arxiv.org/ abs/2002.10291, 2020b.

O. Ljungqvist, K. Bergman, and D. Axehill. Optimization-based motion planning for multi-steered articulated vehicles. Accepted for publication in Proceedings of the 21th IFAC World Congress. Preprint: https://arxiv.org/abs/1912. 06264, 2020c.

J. Löfberg. Minimax approaches to robust model predictive control, volume 812 . Linköping University Electronic Press, 2003.

J. Löfberg. YALMIP: A toolbox for modeling and optimization in MATLAB. In Proceedings of the 2004 IEEE International Symposium on Computer Aided Control Systems Design, pages 284-289, 2004.

A. De Luca, G. Oriolo, and C. Samson. Feedback control of a nonholonomic car-like robot. In Robot motion planning and control, pages 171-253. Springer, 1998.

K. M. Lynch and F. C. Park. Modern Robotics. Cambridge University Press, 2017.

A. Majumdar and R. Tedrake. Funnel libraries for real-time robust feedback motion planning. The International Journal of Robotics Research, 36(8):947-982, 2017.

M. McNaughton, C. Urmson, J. M Dolan, and J-W. Lee. Motion planning for autonomous driving with a conformal spatiotemporal lattice. In Proceedings of the 2011 IEEE/RSJ International conference on Intelligent Robots and Systems, pages 4889-4895, 2011. 
M. M. Michałek. Cascaded approach to the path-following problem for N-trailer robots. In 9th International Workshop on Robot Motion and Control, pages 161-166, 2013a.

M. M. Michałek. Non-minimum-phase property of N-trailer kinematics resulting from off-axle interconnections. International Journal of Control, 86(4):740-758, 2013b.

M. M. Michałek. A highly scalable path-following controller for N-trailers with off-axle hitching. Control Engineering Practice, 29:61-73, 2014.

M. M. Michałek. Cascade-like modular tracking controller for non-standard Ntrailers. IEEE Transactions on Control Systems Technology, 25(2):619-627, 2017.

G. Morrison and D. Cebon. Sideslip estimation for articulated heavy vehicles at the limits of adhesion. Vehicle System Dynamics, 54(11):1601-1628, 2016.

ApS MOSEK. The MOSEK optimization toolbox for MATLAB manual. Version 9.0., 2019. URL http://docs.mosek.com/9.0/toolbox/index.html.

R. M. Murray and S. S. Sastry. Steering nonholonomic systems in chained form. In Proceedings of the 30th IEEE Conference on Decision and Control, pages 1121-1126 vol.2, 1991.

J. Nasir, F. Islam, U. Malik, Y. Ayaz, O. Hasan, M. Khan, and M. S Muhammad. RRT*-SMART: A rapid convergence implementation of RRT. International Journal of Advanced Robotic Systems, 10(7):299, 2013.

I. Nielsen. Structure-Exploiting Numerical Algorithms for Optimal Control. PhD thesis, Linköping University, 2017.

R. Oliveira, P. F Lima, M. Cirillo, J. Mårtensson, and B. Wahlberg. Trajectory generation using sharpness continuous dubins-like paths with applications in control of heavy-duty vehicles. In Proceedings of the 2018 European Control Conference, pages 935-940, 2018.

R. Oliveira, O. Ljungqvist, P. F. Lima, and B. Wahlberg. Optimization-based on-road path planning for articulated vehicles. Accepted for publication in Proceedings of the 21th IFAC World Congress. Preprint: https : //arxiv . org/abs / 2001.06827, 2020a.

R. Oliveira, O. Ljungqvist, P. F. Lima, and B. Wahlberg. A geometric approach to on-road motion planning for long and multi-body heavy-duty vehicles. Accepted for publication in Proceedings of the 31st IEEE Intelligent Vehicles Symposium, 2020 b.

B. Paden, M. Cap, S. Z. Yong, D. Yershov, and E. Frazzoli. A survey of motion planning and control techniques for self-driving urban vehicles. IEEE Transactions on Intelligent Vehicles, 1(1):33-55, 2016. 
G. C. Pereira, P. F. Lima, B. Wahlberg, H. Pettersson, and J. Mårtensson. Linear time-varying robust model predictive control for discrete-time nonlinear systems. In Proceedings of the 2018 IEEE Conference on Decision and Control, pages 2659-2666, 2018.

M. Pivtoraiko and A. Kelly. Kinodynamic motion planning with state lattice motion primitives. In Proceedings of the 2011 IEEE/RSJ International Conference on Intelligent Robots and Systems, pages 2172-2179, 2011.

M. Pivtoraiko, R. A. Knepper, and A. Kelly. Differentially constrained mobile robot motion planning in state lattices. Journal of Field Robotics, 26(3):308 $333,2009$.

M. G Plessen, D. Bernardini, H. Esen, and A. Bemporad. Spatial-based predictive control and geometric corridor planning for adaptive cruise control coupled with obstacle avoidance. IEEE Transactions on Control Systems Technology, 26(1): $38-50,2018$.

S. L Pontryagin. Mathematical theory of optimal processes. Routledge, 2018.

C. Pradalier and K. Usher. Robust trajectory tracking for a reversing tractor trailer. Journal of Field Robotics, 25(6-7):378-399, 2008.

R. Rajamani. Vehicle dynamics and control. Springer Science \& Business Media, 2011.

R. Ravi, K. M Nagpal, and P. P Khargonekar. H-infinity control of linear timevarying systems: A state-space approach. SIAM Journal on Control and Optimization, 29(6):1394-1413, 1991.

J. B Rawlings. Tutorial overview of model predictive control. IEEE Control Systems, 20(3):38-52, 2000.

J. Reeds and L. Shepp. Optimal paths for a car that goes both forwards and backwards. Pacific Journal of Mathematics, 145(2):367-393, 1990.

A. J Rimmer and D. Cebon. Implementation of reversing control on a doubly articulated vehicle. Journal of Dynamic Systems, Measurement, and Control, $139(6), 2017$.

S. Rosbach, V. James, S. Großjohann, S. Homoceanu, and S. Roth. Driving with style: Inverse reinforcement learning in general-purpose planning for automated driving. arXiv preprint arXiv:1905.00229, 2019.

U. Rosolia and F. Borrelli. Learning model predictive control for iterative tasks. a data-driven control framework. IEEE Transactions on Automatic Control, 63 (7):1883-1896, 2017.

U. Rosolia, A. Carvalho, and F. Borrelli. Autonomous racing using learning model predictive control. In Proceedings of the 2017 American Control Conference, pages $5115-5120,2017$. 
P. Rouchon, M. Fliess, J. Levine, and P. Martin. Flatness, motion planning and trailer systems. In Proceedings of 32nd IEEE Conference on Decision and Control, pages 2700-2705 vol.3, 1993.

W. J Rugh and J. S Shamma. Research on gain scheduling. Automatica, 36(10): 1401-1425, 2000.

S. J. Russell and P. Norvig. Artificial intelligence: a modern approach. Malaysia; Pearson Education Limited, 2016.

M. Sampei and K. Furuta. On time scaling for nonlinear systems: Application to linearization. IEEE Transactions on Automatic Control, 31(5):459-462, 1986.

S. Sekhavat, P. Svestka, J-P. Laumond, and M. H. Overmars. Multilevel path planning for nonholonomic robots using semiholonomic subsystems. The International Journal of Robotics Research, 17(8):840-857, 1998.

S. Singh, M. Chen, S. L Herbert, C. J Tomlin, and M. Pavone. Robust tracking with model mismatch for fast and safe planning: an SOS optimization approach. arXiv preprint arXiv:1808.00649, 2018.

I. Skog and P. Händel. In-car positioning and navigation technologies - A survey. IEEE Transactions on Intelligent Transportation Systems, 10(1):4-21, 2009.

O. J. Sørdalen. Conversion of the kinematics of a car with $\mathrm{n}$ trailers into a chained form. In Proceedings of the 1993 IEEE International Conference on Robotics and Automation, pages 382-387, 1993.

M. W. Spong, S. Hutchinson, M. Vidyasagar, et al. Robot modeling and control, volume 3. Wiley New York, 2006.

P. B. Sujit, S. Saripalli, and J. B. Sousa. Unmanned aerial vehicle path following: A survey and analysis of algorithms for fixed-wing unmanned aerial vehicles. IEEE Control Systems Magazine, 34(1):42-59, 2014.

L. Svensson, L. Masson, N. Mohan, E. Ward, A. P. Brenden, L. Feng, and M. Törngren. Safe stop trajectory planning for highly automated vehicles: An optimal control problem formulation. In Proceedings of the 2018 IEEE Intelligent Vehicles Symposium, pages 517-522, 2018.

L. Svensson, M. Bujarbaruah, N. Kapania, and M. Törngren. Adaptive trajectory planning and optimization at limits of handling. arXiv preprint arXiv:1903.04240, 2019.

L. Svensson, M. Bujarbaruah, N. R. Kapania, and M. Törngren. Adaptive trajectory planning and optimization at limits of handling. In Proceedings of the 2019 IEEE/RSJ International Conference on Intelligent Robots and Systems, pages 3942-3948, 2019.

P. Švestka and J. Vleugels. Exact motion planning for tractor-trailer robots. In Proceedings of the 1995 IEEE International Conference on Robotics and Automation, volume 3, pages 2445-2450, 1995. 
M. Tai, J-Y Wang, R. White, and M. Tomizuka. Robust lateral control of heavy duty vehicles. IFAC Proceedings Volumes, 32(2):8309-8314, 1999.

S. Thrun. Toward robotic cars. Communications of the ACM, 53(4):99-106, 2010.

D. Tilbury, R. M. Murray, and S. Sastry. Trajectory generation for the n-trailer problem using Goursat normal form. IEEE Transactions on Automatic Control, 40(5):802-819, 1995.

J. Tumova, G. C Hall, S. Karaman, E. Frazzoli, and D. Rus. Least-violating control strategy synthesis with safety rules. In Proceedings of the 16th international conference on Hybrid systems: computation and control, pages 1-10, 2013.

C. Urmson and R. Simmons. Approaches for heuristically biasing RRT growth. In Proceedings of the 2003 IEEE/RSJ International Conference on Intelligent Robots and Systems, volume 2, pages 1178-1183, 2003.

C. Urmson et al. Autonomous driving in urban environments: Boss and the Urban Challenge. Journal of Field Robotics, 25(8):425-466, 2008.

B. Varga, B. Kulcsar, L. Laine, M. Islam, and B. Nemeth. Robust tracking controller design for active dolly steering. Proceedings of the Institution of $\mathrm{Me}-$ chanical Engineers, Part D: Journal of Automobile Engineering, 232(5):695-706, 2018.

A. Wächter and L. T. Biegler. On the implementation of a primal-dual interior point filter line search algorithm for large-scale nonlinear programming. Mathematical Programming, 106(1):25-57, 2006.

R. S Wallace, A. Stentz, C. E Thorpe, H. P Moravec, W. Whittaker, and T. Kanade. First results in robot road-following. In IJCAI, pages 1089-1095. Citeseer, 1985.

E. Ward, N. Evestedt, D. Axehill, and J. Folkesson. Probabilistic model for interaction aware planning in merge scenarios. IEEE Transactions on Intelligent Vehicles, 2(2):133-146, 2017.

M. Werling, L. Groll, and G. Bretthauer. Invariant trajectory tracking with a fullsize autonomous road vehicle. IEEE Transactions on Robotics, 26(4):758-765, 2010 .

M. Werling, S. Kammel, J. Ziegler, and L. Gröll. Optimal trajectories for timecritical street scenarios using discretized terminal manifolds. The International Journal of Robotics Research, 31(3):346-359, 2012.

M. Werling, P. Reinisch, M. Heidingsfeld, and K. Gresser. Reversing the general one-trailer system: Asymptotic curvature stabilization and path tracking. IEEE Transactions on Intelligent Transportation Systems, 15(2):627-636, 2014.

H. Wolkowicz, R. Saigal, and L. Vandenberghe. Handbook of semidefinite programming: theory, algorithms, and applications, volume 27. Springer Science \& Business Media, 2012. 
World Health Organization. Global status report on road safety. Technical report, 2015 .

K. Zhou, J. Doyle, G. Comstock, Keith, et al. Robust and optimal control, volume 40. Prentice hall New Jersey, 1996.

J. Ziegler and C. Stiller. Spatiotemporal state lattices for fast trajectory planning in dynamic on-road driving scenarios. In Proceedings of the 2019 IEEE/RSJ International Conference on Intelligent Robots and Systems, pages 1879-1884, 2009 . 

Part II

Publications 



\section{Papers}

The papers associated with this thesis have been removed for copyright reasons. For more details about these see:

http://urn.kb.se/resolve?urn=urn:nbn:se:liu:diva-165246 


\section{PhD Dissertations \\ Division of Automatic Control \\ Linköping University}

M. Millnert: Identification and control of systems subject to abrupt changes. Thesis No. 82, 1982. ISBN 91-7372-542-0.

A. J. M. van Overbeek: On-line structure selection for the identification of multivariable systems. Thesis No. 86, 1982. ISBN 91-7372-586-2.

B. Bengtsson: On some control problems for queues. Thesis No. 87, 1982. ISBN 917372-593-5.

S. Ljung: Fast algorithms for integral equations and least squares identification problems. Thesis No. 93, 1983. ISBN 91-7372-641-9.

H. Jonson: A Newton method for solving non-linear optimal control problems with general constraints. Thesis No. 104, 1983. ISBN 91-7372-718-0.

E. Trulsson: Adaptive control based on explicit criterion minimization. Thesis No. 106, 1983. ISBN 91-7372-728-8.

K. Nordström: Uncertainty, robustness and sensitivity reduction in the design of single input control systems. Thesis No. 162, 1987. ISBN 91-7870-170-8.

B. Wahlberg: On the identification and approximation of linear systems. Thesis No. 163, 1987. ISBN 91-7870-175-9.

S. Gunnarsson: Frequency domain aspects of modeling and control in adaptive systems. Thesis No. 194, 1988. ISBN 91-7870-380-8.

A. Isaksson: On system identification in one and two dimensions with signal processing applications. Thesis No. 196, 1988. ISBN 91-7870-383-2.

M. Viberg: Subspace fitting concepts in sensor array processing. Thesis No. 217, 1989. ISBN 91-7870-529-0.

K. Forsman: Constructive commutative algebra in nonlinear control theory. Thesis No. 261, 1991. ISBN 91-7870-827-3.

F. Gustafsson: Estimation of discrete parameters in linear systems. Thesis No. 271, 1992. ISBN 91-7870-876-1.

P. Nagy: Tools for knowledge-based signal processing with applications to system identification. Thesis No. 280, 1992. ISBN 91-7870-962-8.

T. Svensson: Mathematical tools and software for analysis and design of nonlinear control systems. Thesis No. 285, 1992. ISBN 91-7870-989-X.

S. Andersson: On dimension reduction in sensor array signal processing. Thesis No. 290, 1992. ISBN 91-7871-015-4.

H. Hjalmarsson: Aspects on incomplete modeling in system identification. Thesis No. 298, 1993. ISBN 91-7871-070-7.

I. Klein: Automatic synthesis of sequential control schemes. Thesis No. 305, 1993. ISBN 91-7871-090-1.

J.-E. Strömberg: A mode switching modelling philosophy. Thesis No. 353, 1994. ISBN 91-7871-430-3.

K. Wang Chen: Transformation and symbolic calculations in filtering and control. Thesis No. 361, 1994. ISBN 91-7871-467-2.

T. McKelvey: Identification of state-space models from time and frequency data. Thesis No. 380, 1995. ISBN 91-7871-531-8.

J. Sjöberg: Non-linear system identification with neural networks. Thesis No. 381, 1995. ISBN 91-7871-534-2.

R. Germundsson: Symbolic systems - theory, computation and applications. Thesis No. 389, 1995. ISBN 91-7871-578-4. 
P. Pucar: Modeling and segmentation using multiple models. Thesis No. 405, 1995. ISBN 91-7871-627-6.

H. Fortell: Algebraic approaches to normal forms and zero dynamics. Thesis No. 407, 1995. ISBN 91-7871-629-2.

A. Helmersson: Methods for robust gain scheduling. Thesis No. 406, 1995. ISBN 917871-628-4.

P. Lindskog: Methods, algorithms and tools for system identification based on prior knowledge. Thesis No. 436, 1996. ISBN 91-7871-424-8.

J. Gunnarsson: Symbolic methods and tools for discrete event dynamic systems. Thesis No. 477, 1997. ISBN 91-7871-917-8.

M. Jirstrand: Constructive methods for inequality constraints in control. Thesis No. 527, 1998. ISBN 91-7219-187-2.

U. Forssell: Closed-loop identification: Methods, theory, and applications. Thesis No. 566, 1999. ISBN 91-7219-432-4.

A. Stenman: Model on demand: Algorithms, analysis and applications. Thesis No. 571, 1999. ISBN 91-7219-450-2.

N. Bergman: Recursive Bayesian estimation: Navigation and tracking applications. Thesis No. 579, 1999. ISBN 91-7219-473-1.

K. Edström: Switched bond graphs: Simulation and analysis. Thesis No. 586, 1999. ISBN 91-7219-493-6.

M. Larsson: Behavioral and structural model based approaches to discrete diagnosis. Thesis No. 608, 1999. ISBN 91-7219-615-5.

F. Gunnarsson: Power control in cellular radio systems: Analysis, design and estimation. Thesis No. 623, 2000. ISBN 91-7219-689-0.

V. Einarsson: Model checking methods for mode switching systems. Thesis No. 652, 2000. ISBN 91-7219-836-2.

M. Norrlöf: Iterative learning control: Analysis, design, and experiments. Thesis No. 653, 2000. ISBN 91-7219-837-0.

F. Tjärnström: Variance expressions and model reduction in system identification. Thesis No. 730, 2002. ISBN 91-7373-253-2.

J. Löfberg: Minimax approaches to robust model predictive control. Thesis No. 812, 2003. ISBN 91-7373-622-8.

J. Roll: Local and piecewise affine approaches to system identification. Thesis No. 802, 2003. ISBN 91-7373-608-2.

J. Elbornsson: Analysis, estimation and compensation of mismatch effects in A/D converters. Thesis No. 811, 2003. ISBN 91-7373-621-X.

O. Härkegård: Backstepping and control allocation with applications to flight control. Thesis No. 820, 2003. ISBN 91-7373-647-3.

R. Wallin: Optimization algorithms for system analysis and identification. Thesis No. 919, 2004. ISBN 91-85297-19-4.

D. Lindgren: Projection methods for classification and identification. Thesis No. 915, 2005. ISBN 91-85297-06-2.

R. Karlsson: Particle Filtering for Positioning and Tracking Applications. Thesis No. 924, 2005. ISBN 91-85297-34-8.

J. Jansson: Collision Avoidance Theory with Applications to Automotive Collision Mitigation. Thesis No. 950, 2005. ISBN 91-85299-45-6.

E. Geijer Lundin: Uplink Load in CDMA Cellular Radio Systems. Thesis No. 977, 2005. ISBN 91-85457-49-3.

M. Enqvist: Linear Models of Nonlinear Systems. Thesis No. 985, 2005. ISBN 9185457-64-7. 
T. B. Schön: Estimation of Nonlinear Dynamic Systems - Theory and Applications. Thesis No. 998, 2006. ISBN 91-85497-03-7.

I. Lind: Regressor and Structure Selection - Uses of ANOVA in System Identification. Thesis No. 1012, 2006. ISBN 91-85523-98-4.

J. Gillberg: Frequency Domain Identification of Continuous-Time Systems Reconstruction and Robustness. Thesis No. 1031, 2006. ISBN 91-85523-34-8.

M. Gerdin: Identification and Estimation for Models Described by DifferentialAlgebraic Equations. Thesis No. 1046, 2006. ISBN 91-85643-87-4.

C. Grönwall: Ground Object Recognition using Laser Radar Data - Geometric Fitting, Performance Analysis, and Applications. Thesis No. 1055, 2006. ISBN 91-85643-53-X.

A. Eidehall: Tracking and threat assessment for automotive collision avoidance. Thesis No. 1066, 2007. ISBN 91-85643-10-6.

F. Eng: Non-Uniform Sampling in Statistical Signal Processing. Thesis No. 1082, 2007. ISBN 978-91-85715-49-7.

E. Wernholt: Multivariable Frequency-Domain Identification of Industrial Robots. Thesis No. 1138, 2007. ISBN 978-91-85895-72-4.

D. Axehill: Integer Quadratic Programming for Control and Communication. Thesis No. 1158, 2008. ISBN 978-91-85523-03-0.

G. Hendeby: Performance and Implementation Aspects of Nonlinear Filtering. Thesis No. 1161, 2008. ISBN 978-91-7393-979-9.

J. Sjöberg: Optimal Control and Model Reduction of Nonlinear DAE Models. Thesis No. 1166, 2008. ISBN 978-91-7393-964-5.

D. Törnqvist: Estimation and Detection with Applications to Navigation. Thesis No. 1216, 2008. ISBN 978-91-7393-785-6.

P-J. Nordlund: Efficient Estimation and Detection Methods for Airborne Applications. Thesis No. 1231, 2008. ISBN 978-91-7393-720-7.

H. Tidefelt: Differential-algebraic equations and matrix-valued singular perturbation. Thesis No. 1292, 2009. ISBN 978-91-7393-479-4.

H. Ohlsson: Regularization for Sparseness and Smoothness - Applications in System Identification and Signal Processing. Thesis No. 1351, 2010. ISBN 978-91-7393-287-5.

S. Moberg: Modeling and Control of Flexible Manipulators. Thesis No. 1349, 2010. ISBN 978-91-7393-289-9.

J. Wallén: Estimation-based iterative learning control. Thesis No. 1358, 2011. ISBN 978-91-7393-255-4.

J. D. Hol: Sensor Fusion and Calibration of Inertial Sensors, Vision, Ultra-Wideband and GPS. Thesis No. 1368, 2011. ISBN 978-91-7393-197-7.

D. Ankelhed: On the Design of Low Order H-infinity Controllers. Thesis No. 1371, 2011. ISBN 978-91-7393-157-1.

C. Lundquist: Sensor Fusion for Automotive Applications. Thesis No. 1409, 2011. ISBN 978-91-7393-023-9.

P. Skoglar: Tracking and Planning for Surveillance Applications. Thesis No. 1432, 2012. ISBN 978-91-7519-941-2.

K. Granström: Extended target tracking using PHD filters. Thesis No. 1476, 2012. ISBN 978-91-7519-796-8.

C. Lyzell: Structural Reformulations in System Identification. Thesis No. 1475, 2012. ISBN 978-91-7519-800-2.

J. Callmer: Autonomous Localization in Unknown Environments. Thesis No. 1520, 2013. ISBN 978-91-7519-620-6.

D. Petersson: A Nonlinear Optimization Approach to H2-Optimal Modeling and Control. Thesis No. 1528, 2013. ISBN 978-91-7519-567-4. 
Z. Sjanic: Navigation and Mapping for Aerial Vehicles Based on Inertial and Imaging Sensors. Thesis No. 1533, 2013. ISBN 978-91-7519-553-7.

F. Lindsten: Particle Filters and Markov Chains for Learning of Dynamical Systems. Thesis No. 1530, 2013. ISBN 978-91-7519-559-9.

P. Axelsson: Sensor Fusion and Control Applied to Industrial Manipulators. Thesis No. 1585, 2014. ISBN 978-91-7519-368-7.

A. Carvalho Bittencourt: Modeling and Diagnosis of Friction and Wear in Industrial Robots. Thesis No. 1617, 2014. ISBN 978-91-7519-251-2.

M. Skoglund: Inertial Navigation and Mapping for Autonomous Vehicles. Thesis No. 1623, 2014. ISBN 978-91-7519-233-8.

S. Khoshfetrat Pakazad: Divide and Conquer: Distributed Optimization and Robustness Analysis. Thesis No. 1676, 2015. ISBN 978-91-7519-050-1.

T. Ardeshiri: Analytical Approximations for Bayesian Inference. Thesis No. 1710, 2015. ISBN 978-91-7685-930-8.

N. Wahlström: Modeling of Magnetic Fields and Extended Objects for Localization Applications. Thesis No. 1723, 2015. ISBN 978-91-7685-903-2.

J. Dahlin: Accelerating Monte Carlo methods for Bayesian inference in dynamical models. Thesis No. 1754, 2016. ISBN 978-91-7685-797-7.

M. Kok: Probabilistic modeling for sensor fusion with inertial measurements. Thesis No. 1814, 2016. ISBN 978-91-7685-621-5.

J. Linder: Indirect System Identification for Unknown Input Problems: With Applications to Ships. Thesis No. 1829, 2017. ISBN 978-91-7685-588-1.

M. Roth: Advanced Kalman Filtering Approaches to Bayesian State Estimation. Thesis No. 1832, 2017. ISBN 978-91-7685-578-2.

I. Nielsen: Structure-Exploiting Numerical Algorithms for Optimal Control. Thesis No. 1848, 2017. ISBN 978-91-7685-528-7.

D. Simon: Fighter Aircraft Maneuver Limiting Using MPC: Theory and Application. Thesis No. 1881, 2017. ISBN 978-91-7685-450-1.

C. Veibäck: Tracking the Wanders of Nature. Thesis No. 1958, 2018. ISBN 978-917685-200-2.

C. Andersson Naesseth: Machine learning using approximate inference: Variational and sequential Monte Carlo methods. Thesis No. 1969, 2018. ISBN 978-91-7685-161-6.

Y. Jung: Inverse system identification with applications in predistortion. Thesis No. 1966, 2018. ISBN 978-91-7685-171-5.

Y. Zhao: Gaussian Processes for Positioning Using Radio Signal Strength Measurements. Thesis No. 1968, 2019. ISBN 978-91-7685-162-3.

R. Larsson: Flight Test System Identification. Thesis No. 1990, 2019. ISBN 978-917685-070-1.

P. Kasebzadeh: Learning Human Gait. Thesis No. 2012, 2019. ISBN 978-91-7519-0143.

K. Radnosrati: Time of flight estimation for radio network positioning. Thesis No. 2054, 2020. ISBN 978-91-7929-884-5. 


\section{FACULTY OF SCIENCE AND ENGINEERING}

Linköping Studies in Science and Technology, Dissertations No. 2070, 2020 Department of Electrical Engineering

Linköping University

SE-581 83 Linköping, Sweden

www.liu.se

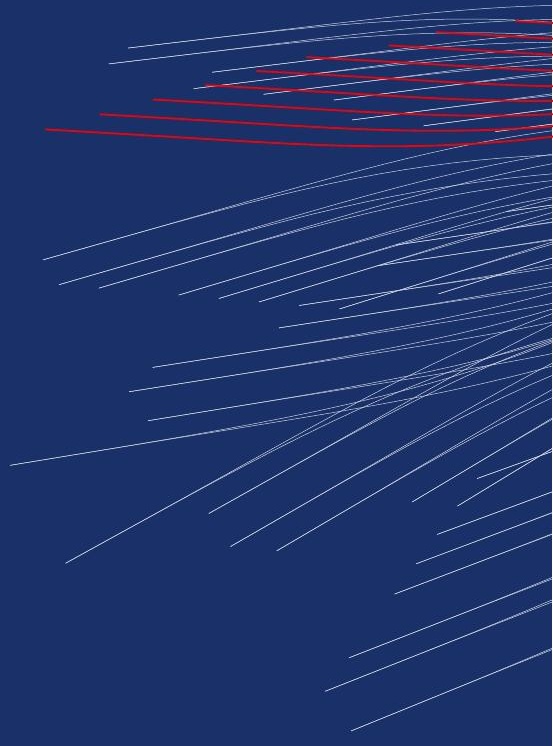

